\title{
Organocatalytic Asymmetric Synthesis of Spirooxindole Embedded Oxazolidines
}

Chandrakanta Parida, Buddhadeb Mondal, Animesh Ghosh and Subhas Chandra Pan* Department of Chemistry, Indian Institute of Technology Guwahati,

North Guwahati, Assam, 781039

Email id: span@iitg.ac.in

\section{Table of contents}

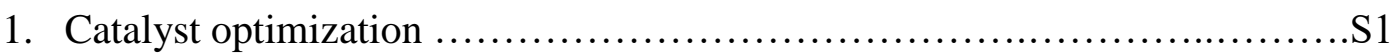

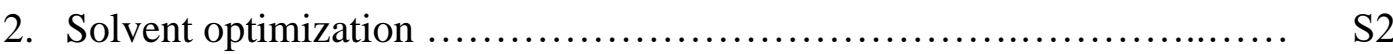

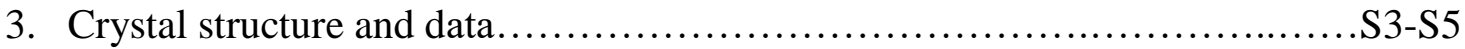

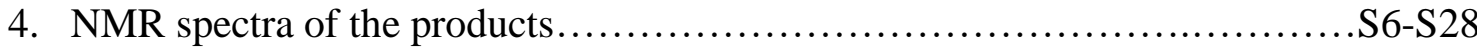



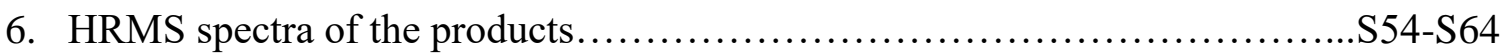


1. Catalyst optimization for tert-butyl (3R,4'R)-1-benzyl-2-oxo-4'-(2-oxo-2 phenylethyl)spiro[indoline-3,2'-oxazolidine]-3'-carboxylate:<smiles>CN(C)[C@H]1CCCC[C@H]1NC(=S)Nc1cc(C(F)(F)F)cc(C(F)(F)F)c1</smiles><smiles>C=CC1CC2CCC1N2[C](NC(=S)N[Ga])c1ccnc2ccc(OC)cc12</smiles>

II



III<smiles>CN[C@H]1CCCC[C@H]1Nc1c(Nc2cc(C(F)(F)F)cc(C(F)(F)F)c2)c(=O)c1=O</smiles>

$\mathrm{Ar}=3,5\left(\mathrm{CF}_{3}\right)_{2} \mathrm{C}_{6} \mathrm{H}_{3}$

IV<smiles>[R][R]Nc1c(N[C@@H](c2ccnc3ccc([R])cc23)C2(C)CC3CCN2CC3C=C)c(=O)c1=O</smiles>

$\mathrm{R}=\mathrm{H}, \mathrm{V}$

$\mathrm{R}=\mathrm{OMe}, \mathrm{VI}$

7.<smiles>CC(C)(C)N=C1C(=O)N(Cc2ccccc2)c2ccccc21</smiles>

1a<smiles>O=C(/C=C/CO)c1ccccc1</smiles>

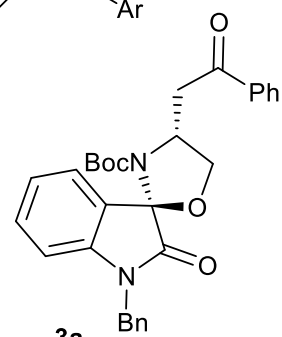

3a $\mathrm{Bn}$

8.

\begin{tabular}{|c|c|c|c|c|}
\hline Entry $^{[\mathrm{a}]}$ & catalyst & $\operatorname{yield}(\%)^{[\mathrm{b}]}$ & $\mathrm{dr}^{[\mathrm{c}]}$ & $\mathrm{ee}(\%)^{[\mathrm{c}]}$ \\
\hline 1 & I & 72 & $1.1: 1$ & 88 \\
\hline 2 & II & 75 & $1.5: 1$ & 95 \\
\hline 3 & III & 40 & $1.1: 1$ & 68 \\
\hline 4 & IV & 67 & $3.9: 1$ & 97 \\
\hline 5 & V & 83 & $\mathbf{3 . 6 : 1}$ & $>99$ \\
\hline $\mathbf{6}$ & VI & $\mathbf{8 5}$ & & $>99$ \\
\hline
\end{tabular}

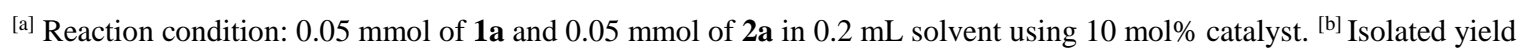
after silica gel column chromatography. ${ }^{[c]}$ Determined by ${ }^{1} \mathrm{H}$ NMR. ${ }^{[\mathrm{d}]}$ Determined by HPLC and of the major diastereomers. 
2. Solvent optimization for tert-butyl (3R,4'R)-1-benzyl-2-oxo-4'-(2-oxo-2 phenylethyl)spiro[indoline-3,2'-oxazolidine]-3'-carboxylate:



9.

$1 \mathrm{a}$

$2 a$

3a Bn

\begin{tabular}{|c|c|c|c|c|}
\hline Entry $^{[\mathrm{a}]}$ & solvent & $\begin{array}{c}\text { yield }^{[\mathrm{b}]} \\
(\%)\end{array}$ & $\mathrm{dr}^{[\mathrm{c}]}$ & $\mathrm{ee}^{[\mathrm{c}]}$ \\
\hline 1 & $\mathrm{PhCF}_{3}$ & 80 & $3.5: 1$ & $>99$ \\
\hline 2 & $\mathrm{xylene}$ & 81 & $3.5: 1$ & $>99$ \\
\hline 3 & $\mathrm{CH}_{2} \mathrm{Cl}_{2}$ & 74 & $2.6: 1$ & $>99$ \\
\hline 4 & $\mathrm{MTBE}$ & 71 & $2.8: 1$ & $>99$ \\
\hline
\end{tabular}


after silica gel column chromatography. ${ }^{[c]}$ Determined by ${ }^{1} \mathrm{H}$ NMR. ${ }^{[\mathrm{d}]}$ Determined by HPLC and of the major diastereomers.

\section{Single crystal $X$-ray diffraction analysis of 3p:}

\section{Instrument used for the crystal measurement:}

BRUKER D8 VENTURE SC-XRD system is equipped with dual X-ray sources (Mo and Cu), Photon II detector, APEX3 and SHEXLTL softwares.

\section{Method for crystal growth:}

Dissolved 50mg of compound $3 \mathrm{p}$ in $1 \mathrm{ml}$ hexane $+1 \mathrm{ml}$ ethyl acetate $+1 \mathrm{ml}$ dicholomethane, kept it for slow evaporation.

Ellipsoid contour \% probability levels in the caption for the image of the structure is 50 .

CCDC No.

Empirical formula

Formula weight

Crystal habit, colour

Crystal size, $\mathrm{mm}^{3}$

Temperature, $T$

Wavelength, $\lambda(\AA)$
2058339

$\mathrm{C}_{30} \mathrm{H}_{29} \mathrm{BrN}_{2} \mathrm{O}_{5}$

576.12

block / white

$0.36 \times 0.33 \times 0.33$

296 K

0.71073 
Crystal system

Space group

Unit cell dimensions

Theta $(\max )=25.048$

Data completeness $=1.87 / 0.99$

$\mathrm{R}($ reflections $)=0.0406(7139)$

$w R 2($ reflections $)=0.0967(9735)$
Monoclinic

'C 2'

$a=21.999(4) b=9.9929(14) c=26.101(5)$

alpha $=90$ beta $=105.083(6)$ gamma $=90$

Datablock CKP_6Br_SCP_0m_a - ellipsoid plot






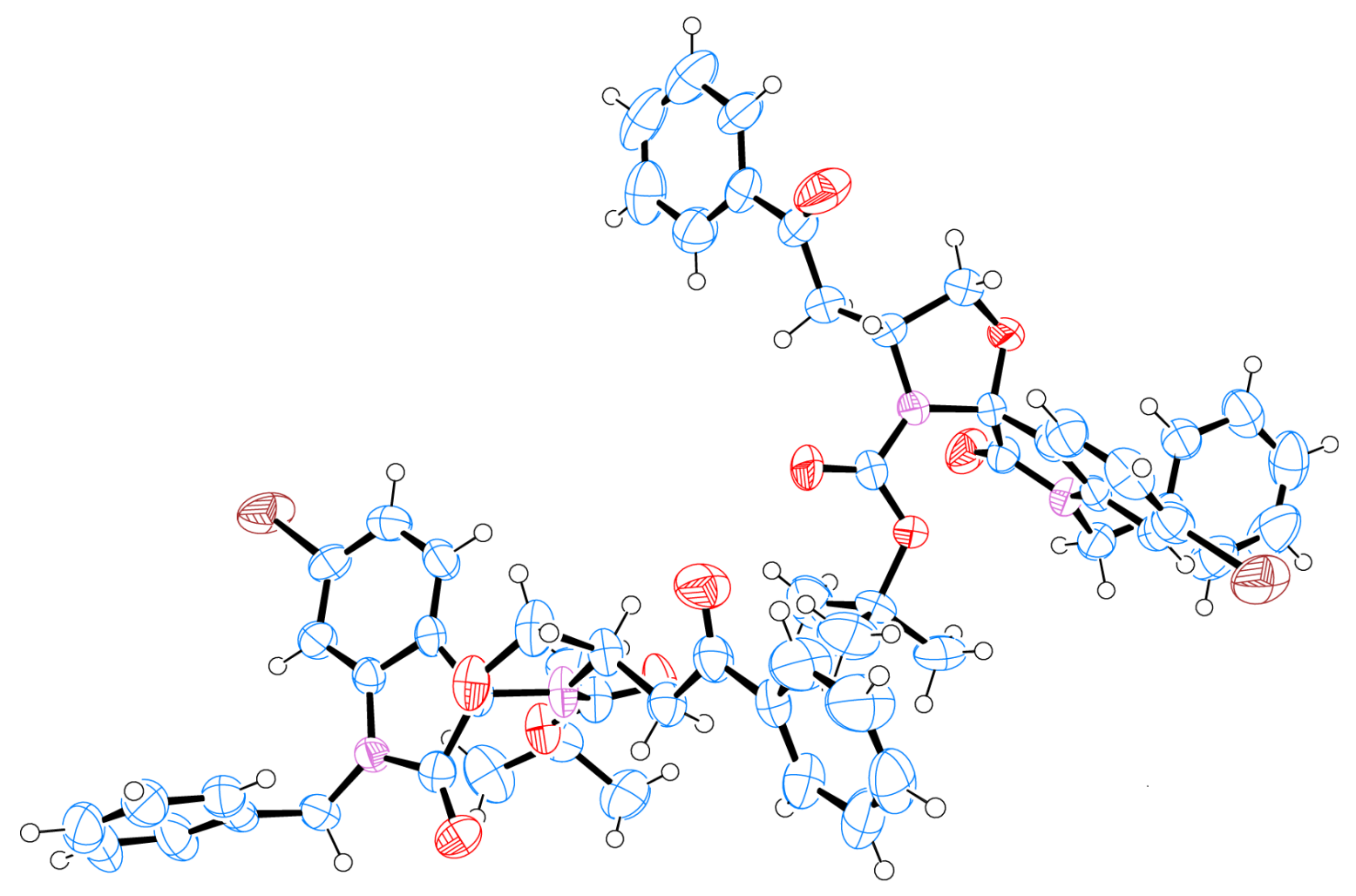

ORTEP DIAGRAM 


\section{NMR spectra of the products:}

${ }^{1} \mathrm{H}$ NMR of $3 \mathrm{a}\left(600 \mathrm{MHz}, \mathrm{CDCl}_{3}\right)$

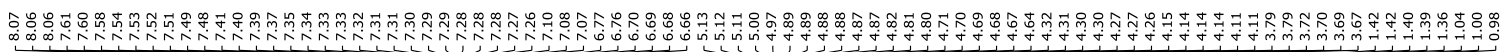

<smiles>O=C(C[C@@H]1COC2(C(=O)N(Cc3ccccc3)c3ccccc32)N1C(=O)c1ccccc1)c1ccccc1</smiles>

$3 a$

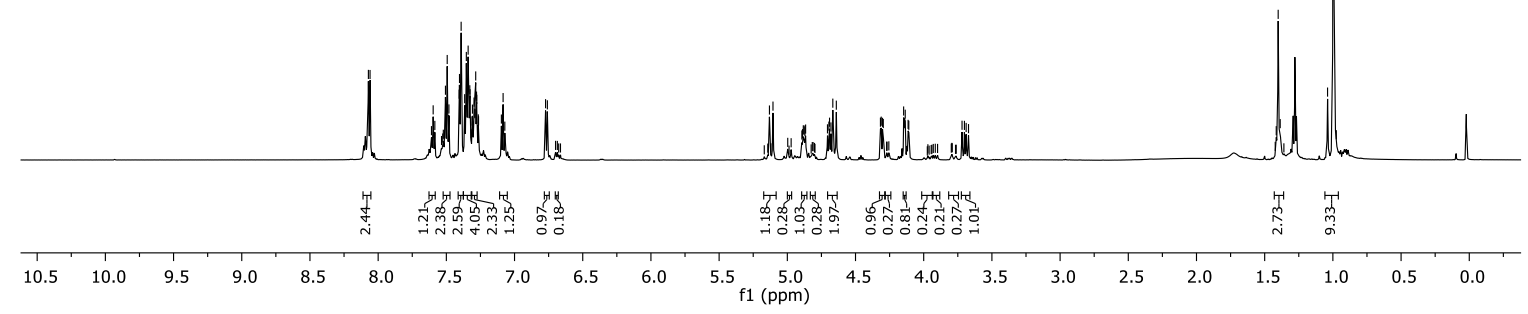

${ }^{13} \mathrm{C}\left\{{ }^{1} \mathrm{H}\right\} \mathrm{NMR}$ of $3 \mathrm{a}\left(151 \mathrm{MHz}, \mathrm{CDCl}_{3}\right)$



${ }^{1} \mathrm{H}$ NMR of $3 \mathrm{~b}\left(400 \mathrm{MHz}, \mathrm{CDCl}_{3}\right)$

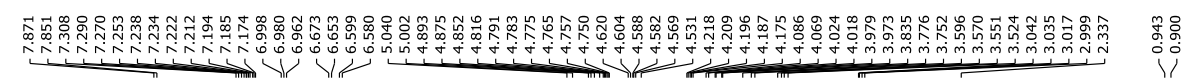

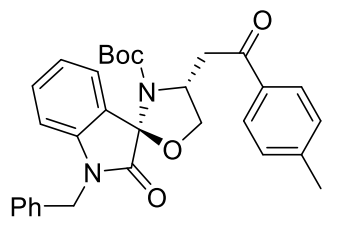

$3 \mathbf{b}$



${ }^{13} \mathrm{C}\left\{{ }^{1} \mathrm{H}\right\}$ NMR of $3 \mathrm{~b}\left(101 \mathrm{MHz}, \mathrm{CDCl}_{3}\right)$

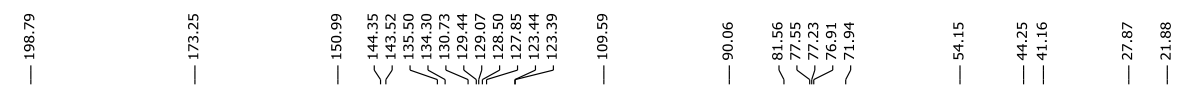



3b



$\begin{array}{llllllllllllllllllllllllll}210 & 200 & 190 & 180 & 170 & 160 & 150 & 140 & 130 & 120 & 110 & 100 & 90 & 80 & 70 & 60 & 50 & 40 & 30 & 20 & 10 & 0 & -10\end{array}$ 
${ }^{1} \mathrm{H}$ NMR of $3 \mathrm{c}\left(400 \mathrm{MHz}, \mathrm{CDCl}_{3}\right)$

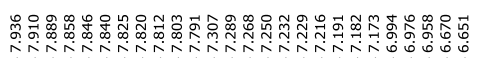

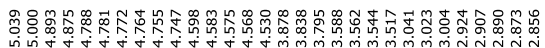

บับ

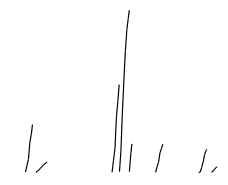

$1,1 \int-1,1,-1$
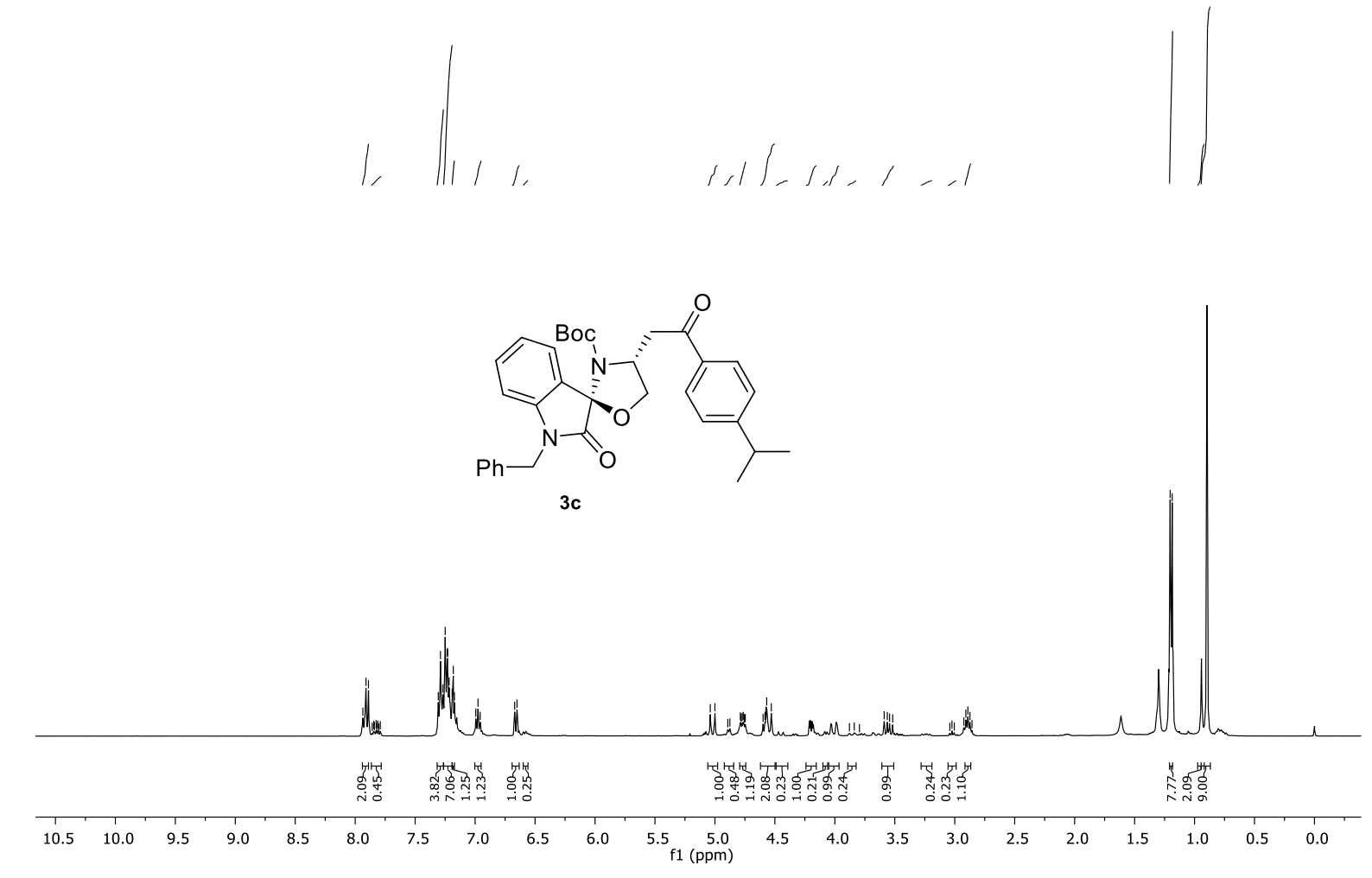

$3 c$

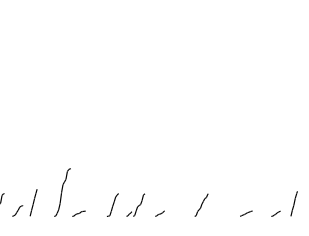

$$
1
$$

\section{${ }^{13} \mathrm{C}\left\{{ }^{1} \mathrm{H}\right\}$ NMR of $3 \mathrm{c}\left(101 \mathrm{MHz}, \mathrm{CDCl}_{3}\right)$}





$\begin{array}{lllllllllll}210 & 200 & 190 & 180 & 170 & 160 & 150 & 140 & 130 & 120 & 110 \\ \mathrm{f} 1(\mathrm{ppm}) & 100\end{array}$ 
${ }^{1} \mathrm{H}$ NMR of $3 \mathrm{~d}\left(400 \mathrm{MHz}, \mathrm{CDCl}_{3}\right)$





\section{${ }^{13} \mathrm{C}\left\{{ }^{1} \mathrm{H}\right\}$ NMR of 3d (101 MHz, $\left.\mathrm{CDCl}_{3}\right)$}

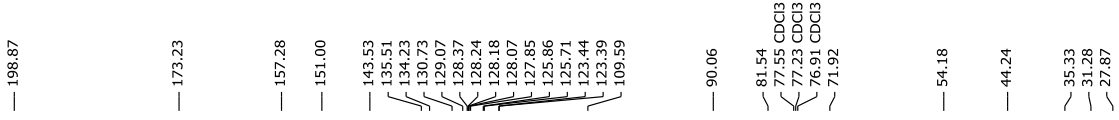



$\begin{array}{lllllllllll}210 & 200 & 190 & 180 & 170 & 160 & 150 & 140 & 130 & 120 & 110 \begin{array}{c}100 \\ \mathrm{f} 1(\mathrm{ppm})\end{array}\end{array}$ 
${ }^{1} \mathrm{H}$ NMR of $3 \mathrm{e}\left(500 \mathrm{MHz}, \mathrm{CDCl}_{3}\right)$

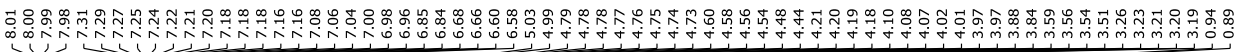

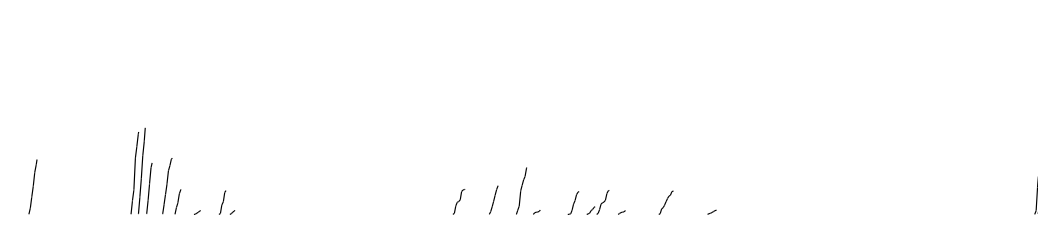<smiles>CN1CC(COCCc2ccccc2)(CC(=O)c2ccc(F)cc2)c2ccccc21</smiles>

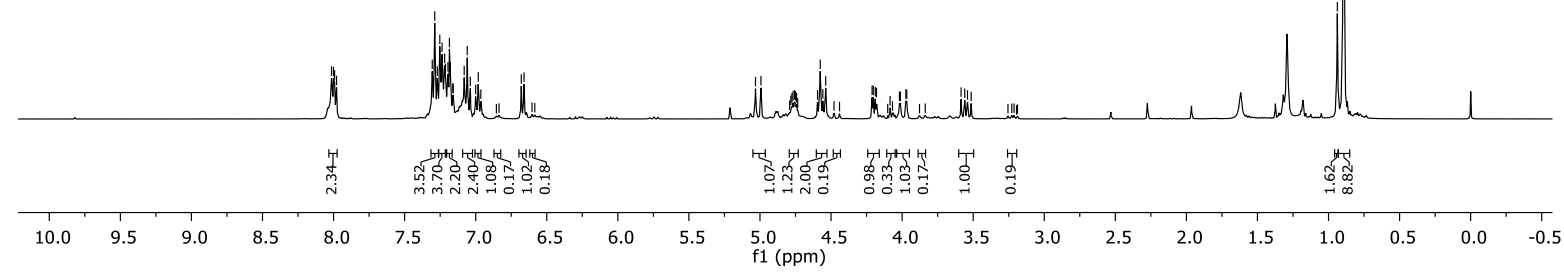

${ }^{13} \mathrm{C}\left\{{ }^{1} \mathrm{H}\right\}$ NMR of $3 \mathrm{e}\left(151 \mathrm{MHz}, \mathrm{CDCl}_{3}\right)$

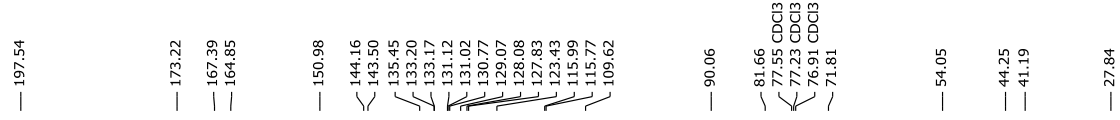
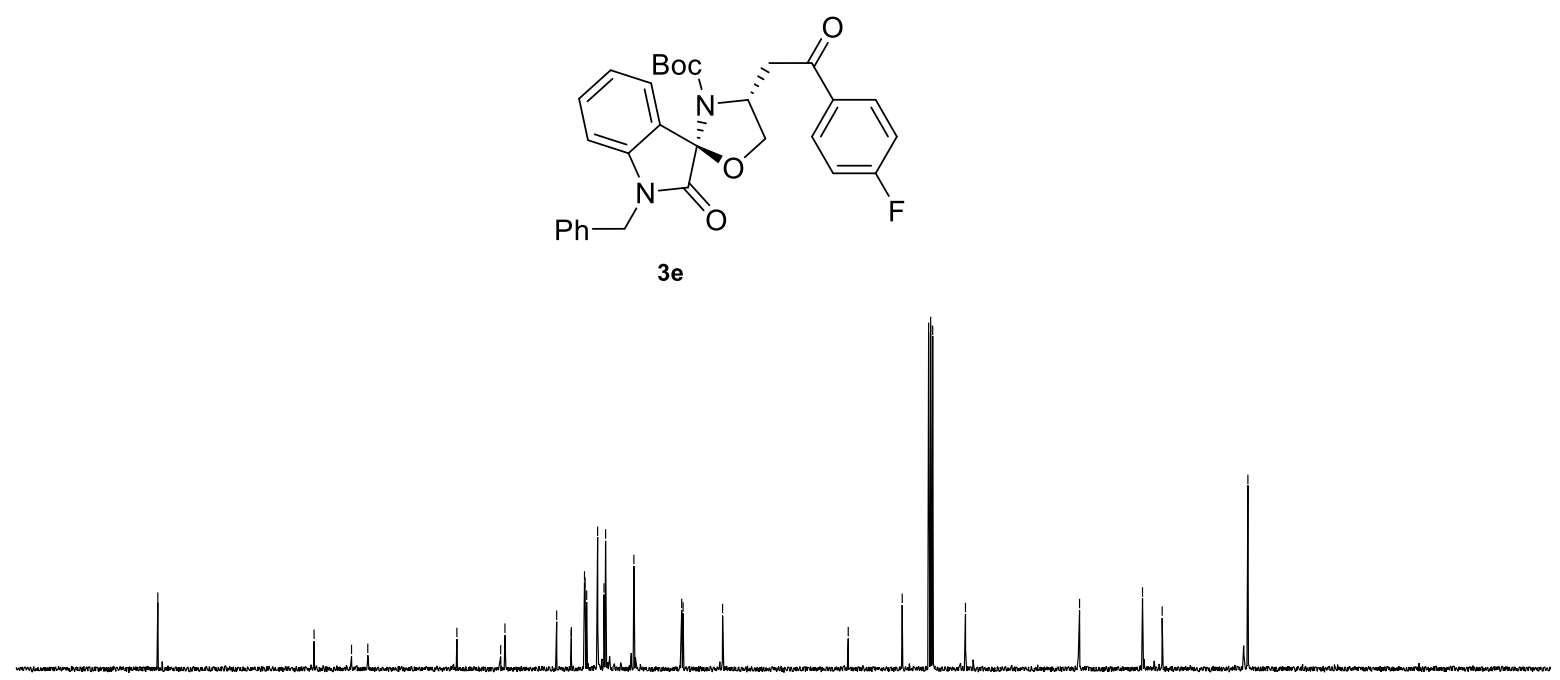

$\begin{array}{lllllllllllllllllllllllllllll}210 & 200 & 190 & 180 & 170 & 160 & 150 & 140 & 130 & 120 & 110 & 100 & 90 & 80 & 70 & 60 & 50 & 40 & 30 & 20 & 10 & 0 & -10\end{array}$ 
${ }^{1} \mathrm{H}$ NMR of $3 f\left(400 \mathrm{MHz}, \mathrm{CDCl}_{3}\right)$

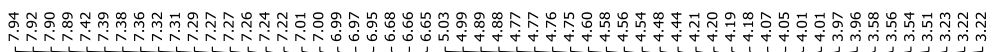

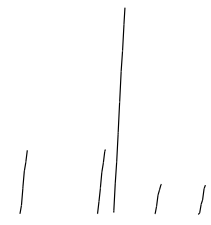

$1,1 /, 11,1$,

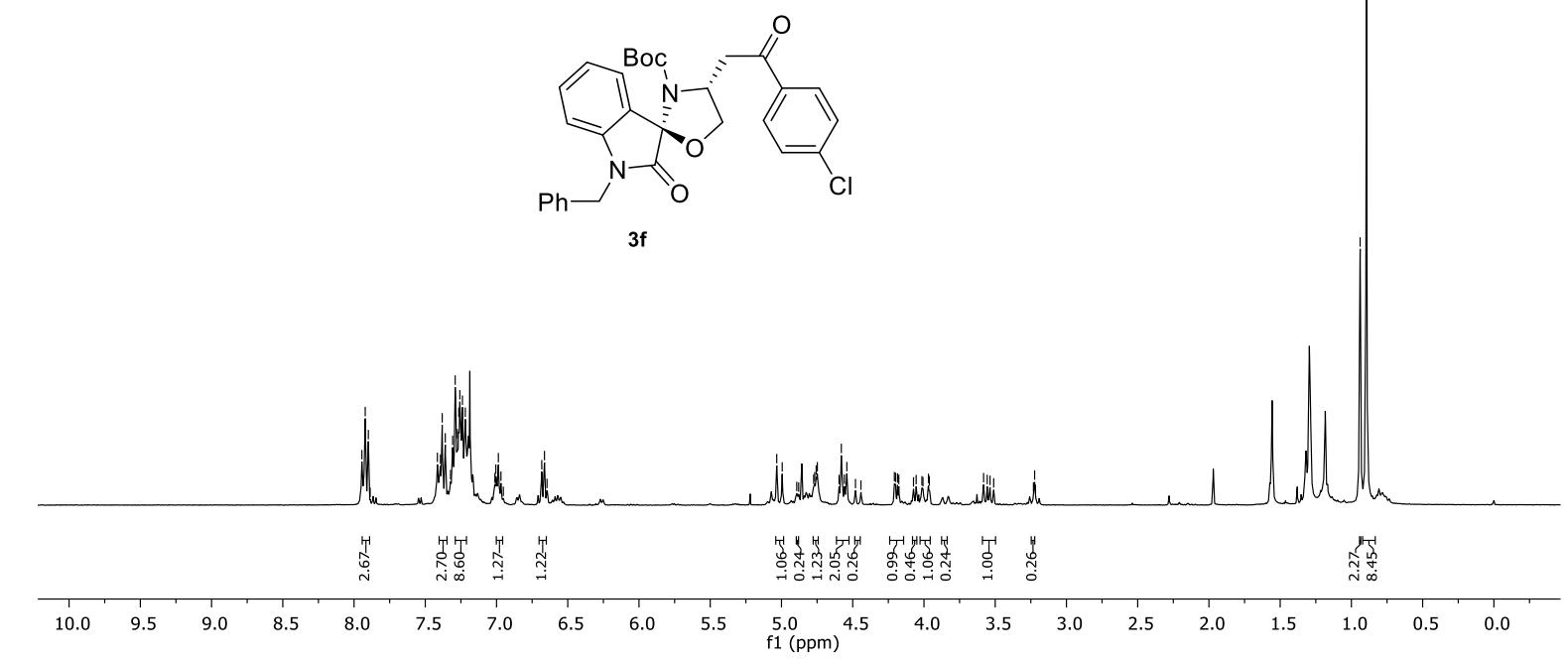

$3 f$

\section{${ }^{13} \mathrm{C}\left\{{ }^{1} \mathrm{H}\right\}$ NMR of $3 f\left(151 \mathrm{MHz}, \mathrm{CDCl}_{3}\right)$}

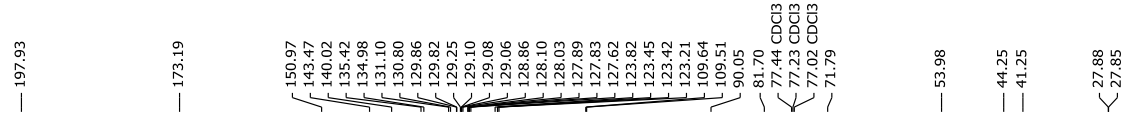

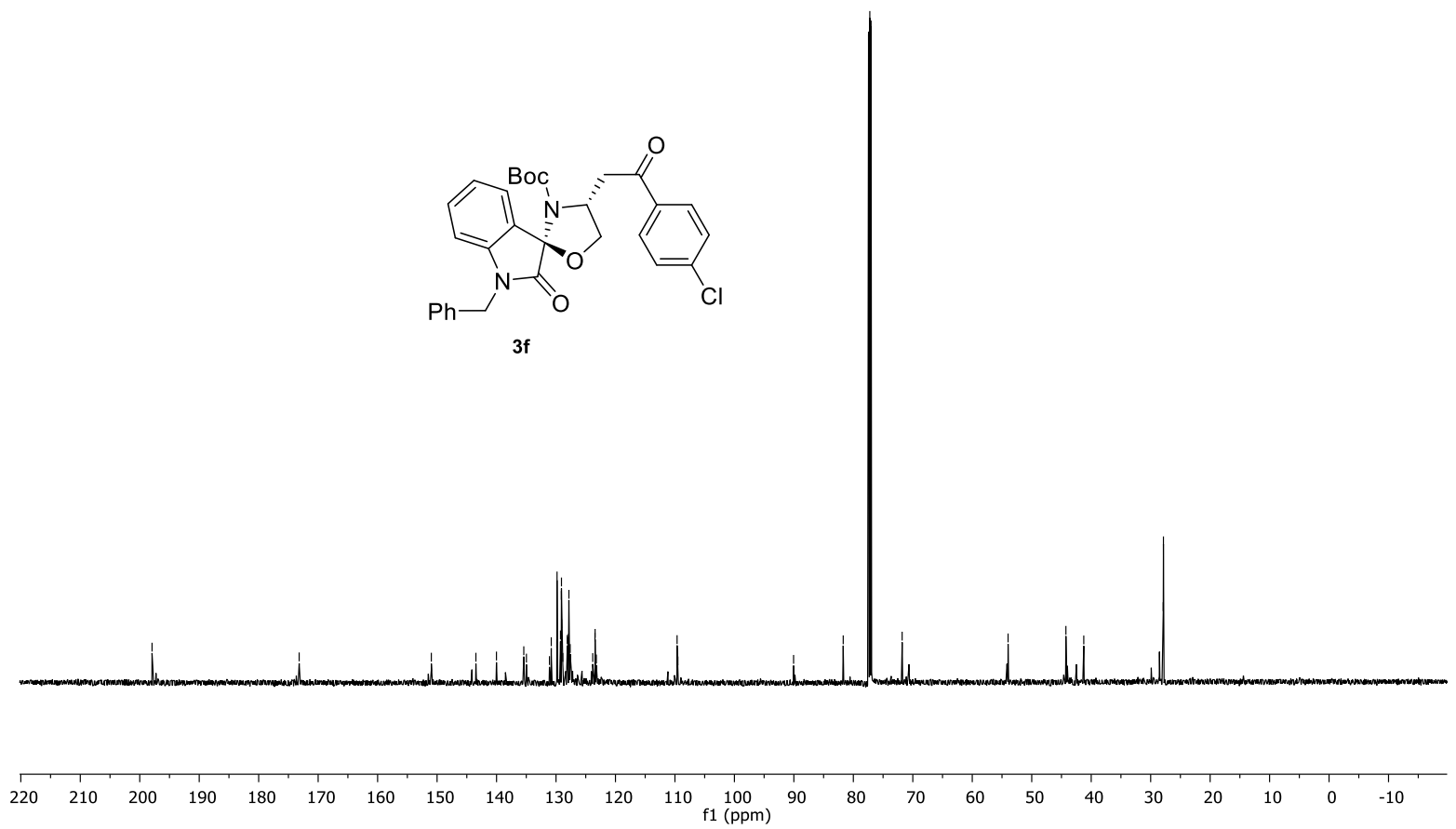


${ }^{1} \mathrm{H}$ NMR of $3 \mathrm{~g}\left(400 \mathrm{MHz}, \mathrm{CDCl}_{3}\right)$

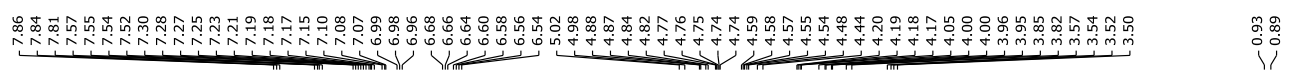

1|| $1 / 1$,

$f, 1, j, f_{1,-}$

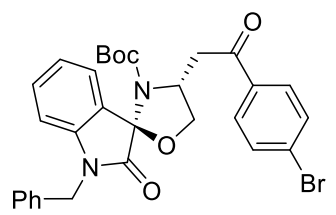

$3 g$

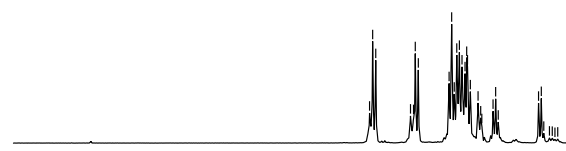





${ }^{13} \mathrm{C}\left\{{ }^{1} \mathrm{H}\right\}$ NMR of $3 g\left(101 \mathrm{MHz}, \mathrm{CDCl}_{3}\right)$

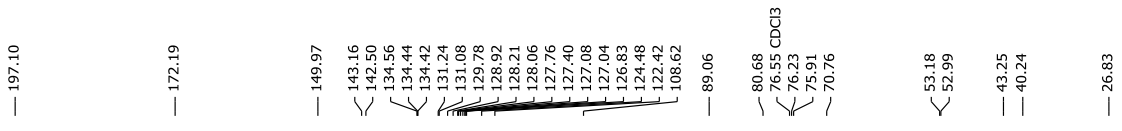
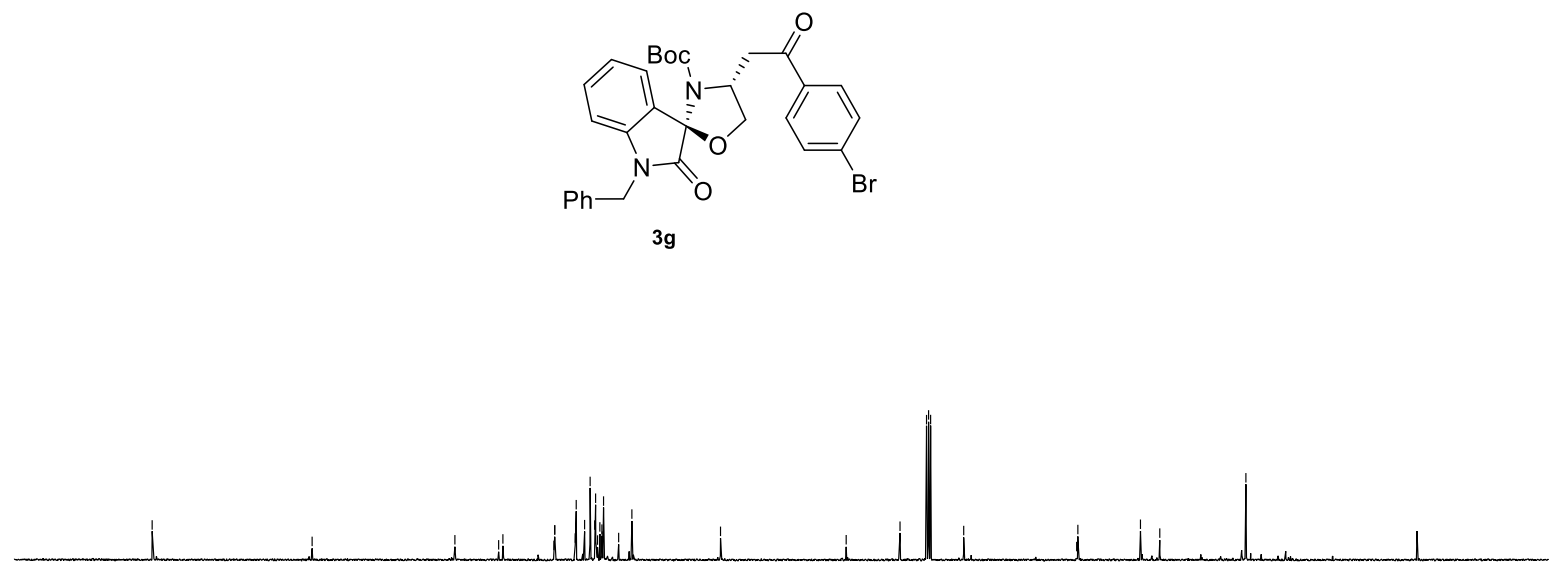

$\begin{array}{llllllllllllllllllllllllllllllllll}210 & 200 & 190 & 180 & 170 & 160 & 150 & 140 & 130 & 120 & 110 & 100 & 90 & 80 & 70 & 60 & 50 & 40 & 30 & 20 & 10 & 0 & -10 & -20\end{array}$ 
${ }^{1} \mathrm{H}$ NMR of $3 \mathrm{~h}\left(400 \mathrm{MHz}, \mathrm{CDCl}_{3}\right)$

<smiles>Cc1cccc(C(=O)C[C@@H]2COC3(O2)c2ccccc2N3Cc2ccccc2)c1</smiles>

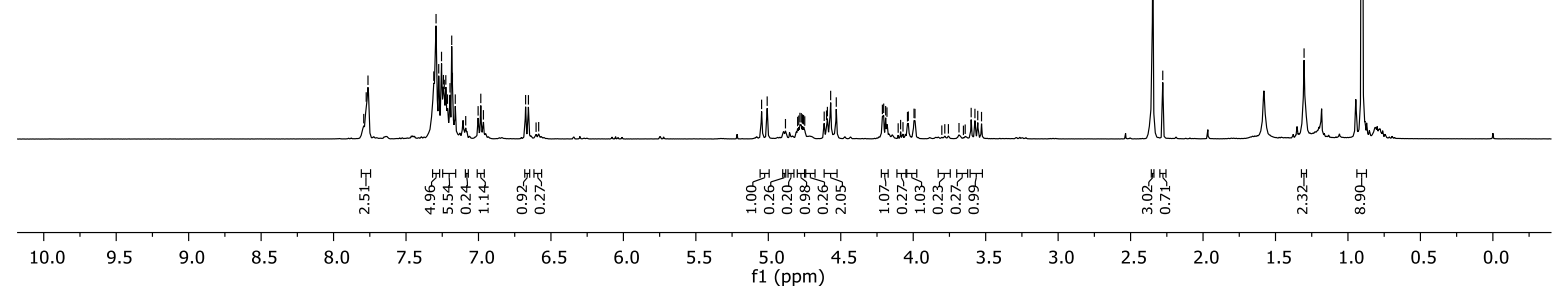

\section{${ }^{13} \mathrm{C}\left\{{ }^{1} \mathrm{H}\right\}$ NMR of $3 \mathrm{~h}\left(101 \mathrm{MHz}, \mathrm{CDCl}_{3}\right)$}

\begin{tabular}{|c|c|c|c|c|c|c|c|}
\hline 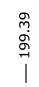 & $\stackrel{\substack{n \\
\stackrel{N}{N}}}{\mid}$ &  & $\begin{array}{l}\infty \\
\stackrel{0}{0} \\
1\end{array}$ &  & $\stackrel{\sim}{\stackrel{\sim}{5}}$ &  & $\stackrel{\substack{\infty \\
\sim}}{\sim}$ \\
\hline
\end{tabular}
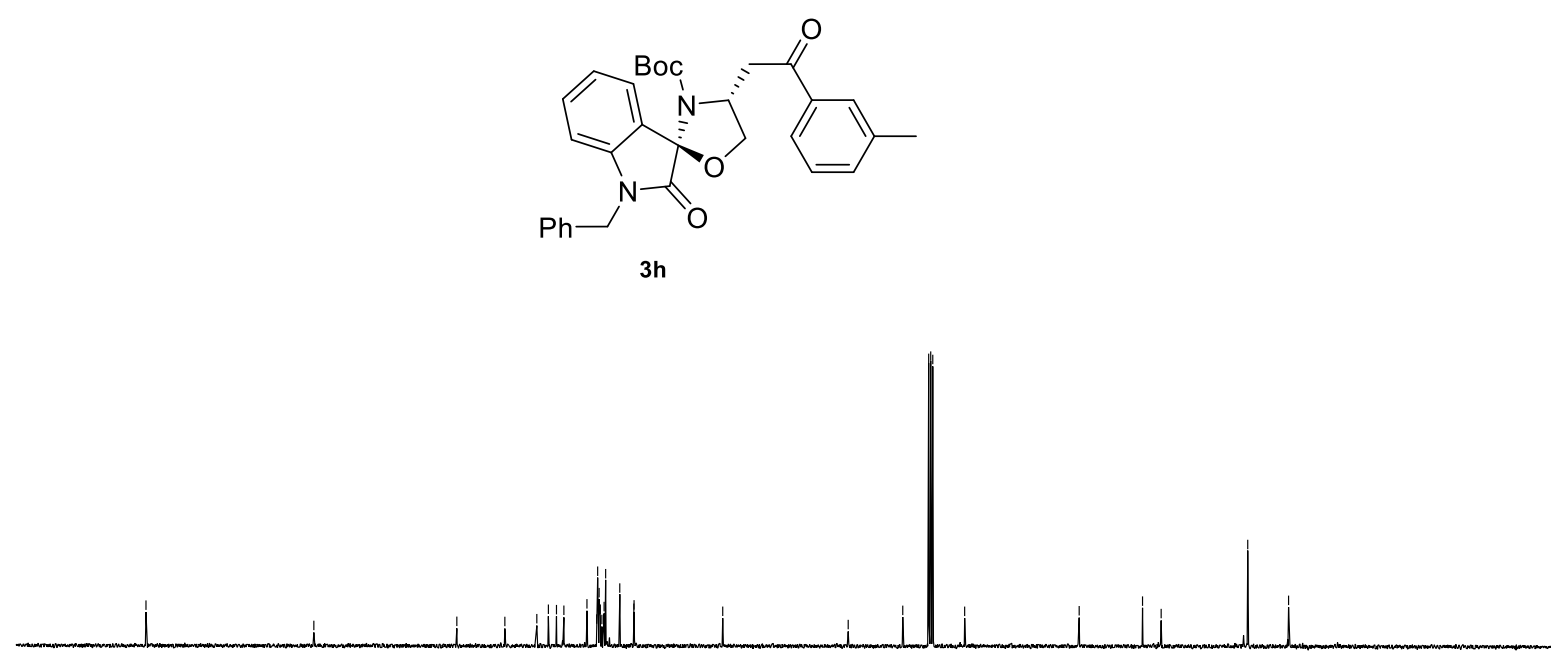

$\begin{array}{llllllllllllllllllllllllllll}210 & 200 & 190 & 180 & 170 & 160 & 150 & 140 & 130 & 120 & 110 & 100 & 90 & 80 & 70 & 60 & 50 & 40 & 30 & 20 & 10 & 0 & -10\end{array}$ 
${ }^{1} \mathrm{H}$ NMR of $3 \mathrm{i}\left(500 \mathrm{MHz}, \mathrm{CDCl}_{3}\right)$


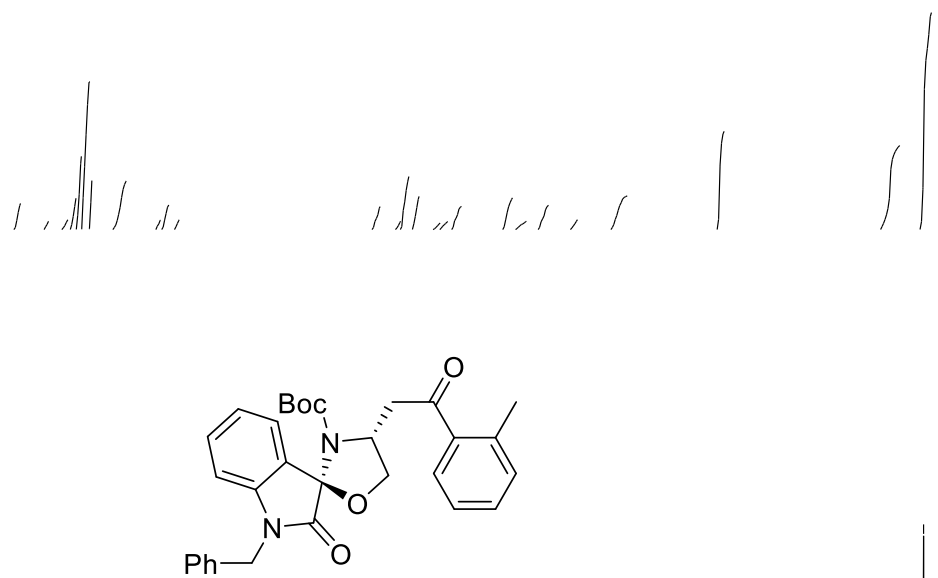

$3 \mathbf{i}$

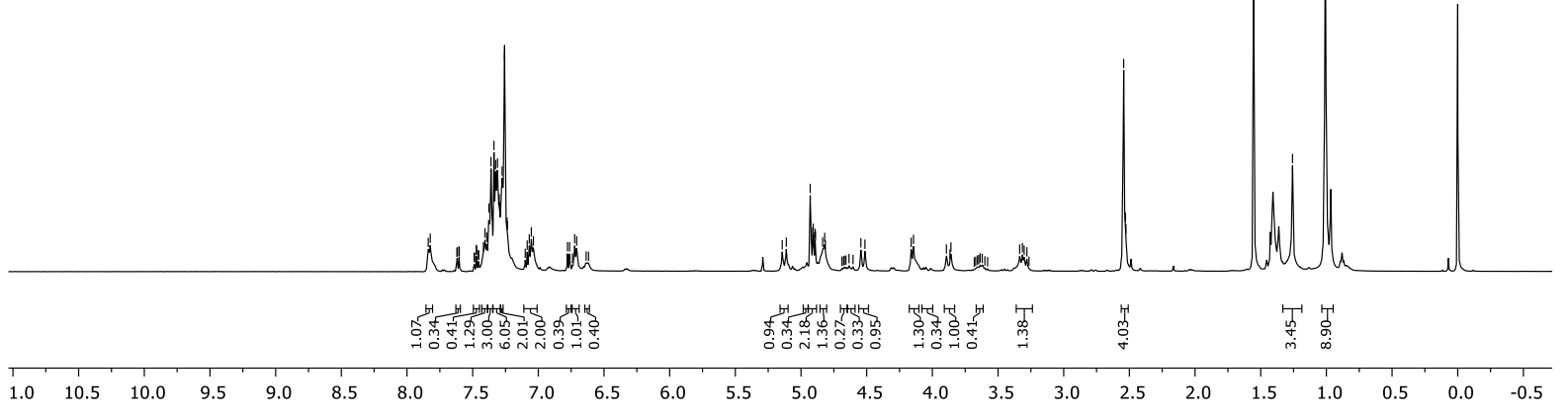

${ }^{13} \mathrm{C}\left\{{ }^{1} \mathrm{H}\right\}$ NMR of $3 \mathrm{i}\left(101 \mathrm{MHz}, \mathrm{CDCl}_{3}\right)$

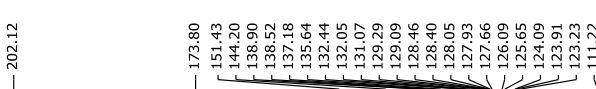
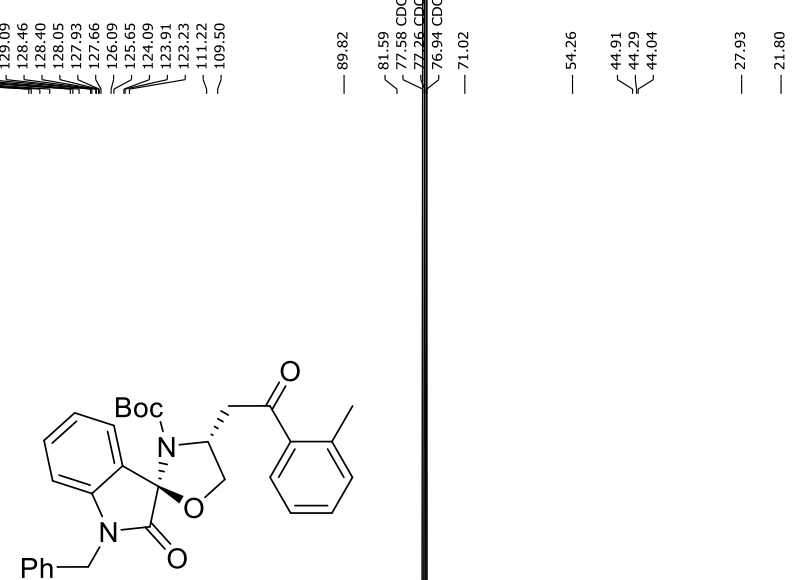

$3 \mathbf{i}$

$\begin{array}{lllllllllllllllllllllllllllll}210 & 200 & 190 & 180 & 170 & 160 & 150 & 140 & 130 & 120 & 110 & 100 & 90 & 80 & 70 & 60 & 50 & 40 & 30 & 20 & 10 & 0 & -10\end{array}$ 
${ }^{1} \mathrm{H}$ NMR of jj (500 MHz, 3$)$

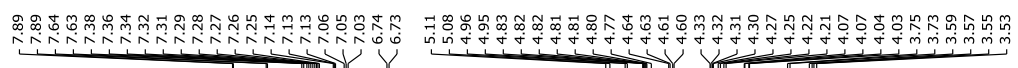

$\stackrel{\substack{0 \\ 1}}{i}$


$3 \mathbf{j}$

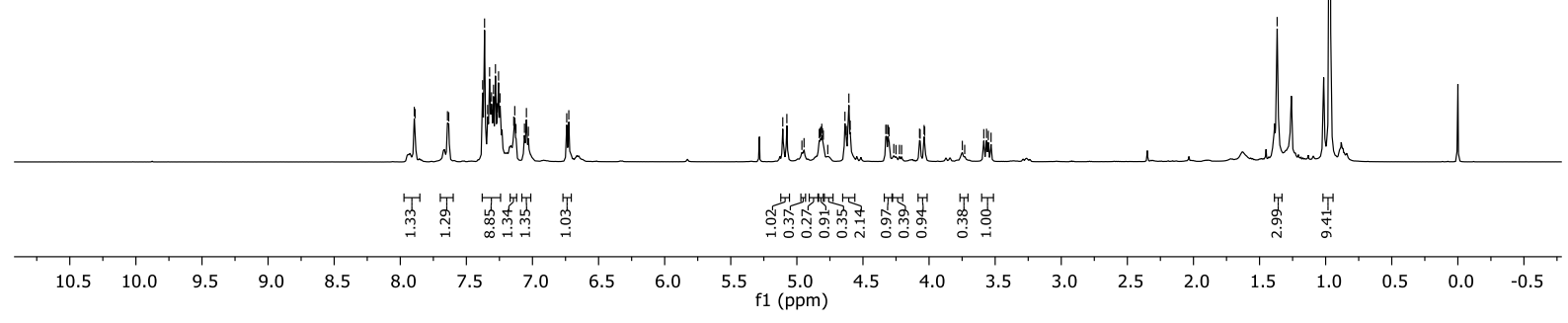

${ }^{13} \mathrm{C}\left\{{ }^{1} \mathrm{H}\right\}$ NMR of $3 \mathrm{j}\left(101 \mathrm{MHz}, \mathrm{CDCl}_{3}\right)$
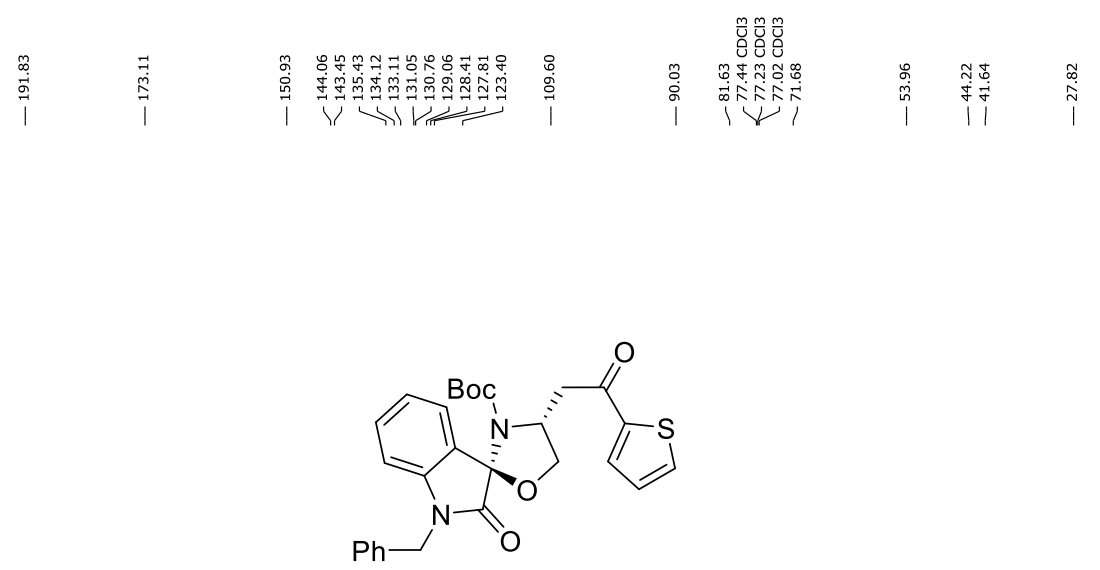

jj
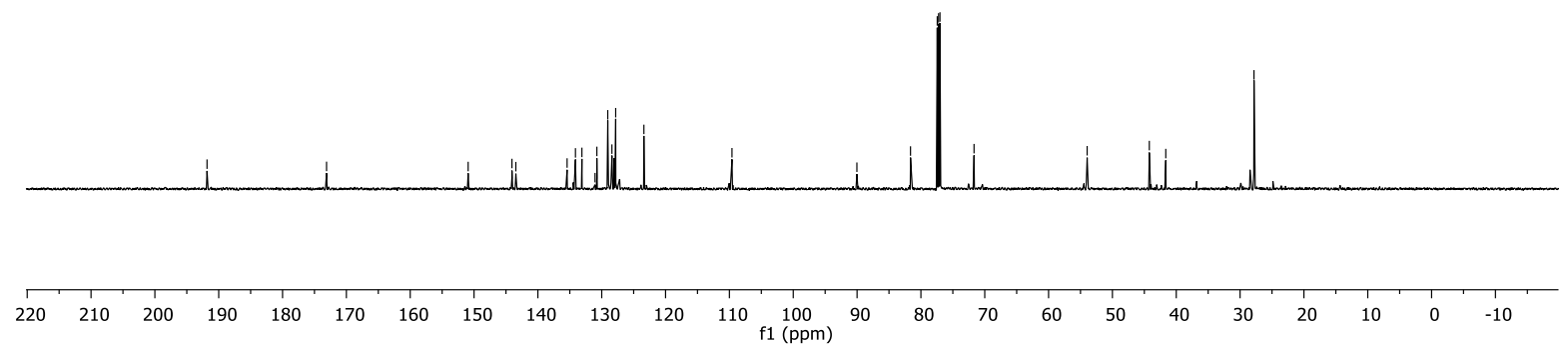

S15 
${ }^{1} \mathrm{H}$ NMR of 3k (500 $\left.\mathrm{MHz}, \mathrm{CDCl}_{3}\right)$

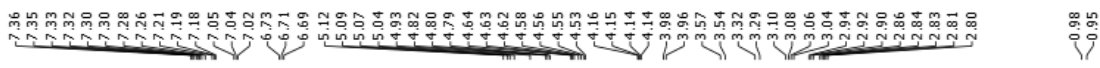
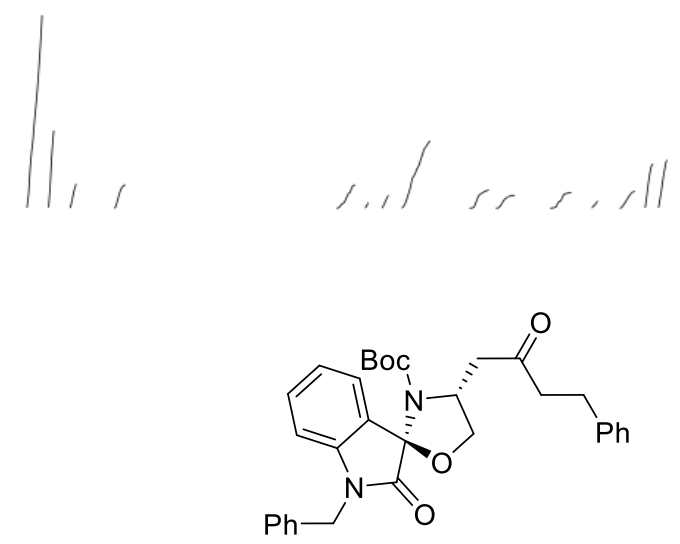

$3 \mathbf{k}$

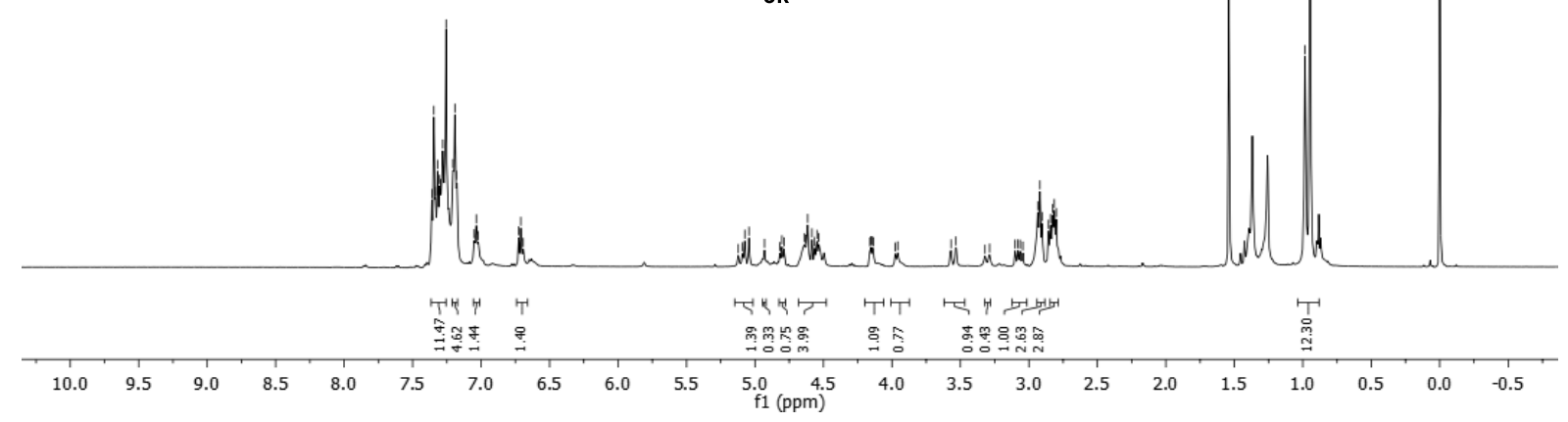

${ }^{13} \mathrm{C}\left\{{ }^{1} \mathrm{H}\right\}$ NMR of $3 \mathrm{k}\left(101 \mathrm{MHz}, \mathrm{CDCl}_{3}\right)$

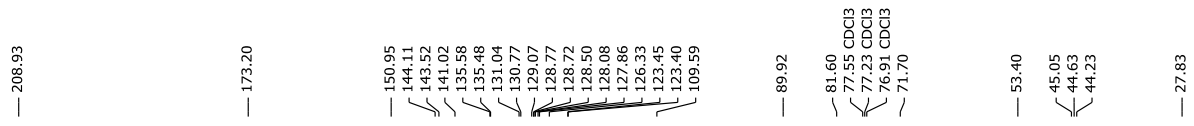

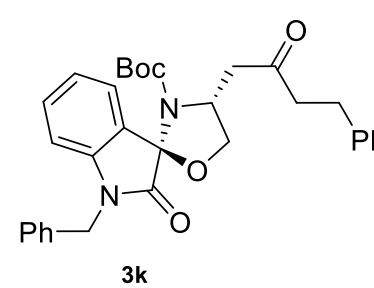

$3 \mathbf{k}$

$\begin{array}{lllllllllll}210 & 200 & 190 & 180 & 170 & 160 & 150 & 140 & 130 & 120 & 110 \begin{array}{c}100 \\ \mathrm{f} 1(\mathrm{ppm})\end{array}\end{array}$ 
${ }^{1} \mathrm{H}$ NMR of 31 (600 MHz, $\left.\mathrm{CDCl}_{3}\right)$

范

$\stackrel{\circ}{\circ}$

等

$\int 1 / \int_{1,1111} \quad 11,111,1,1 / 1$<smiles>COc1ccc2c(c1)C1(OC[C@H](CC(=O)c3ccccc3)C1=O)N(Cc1ccccc1)C2=O</smiles>

31

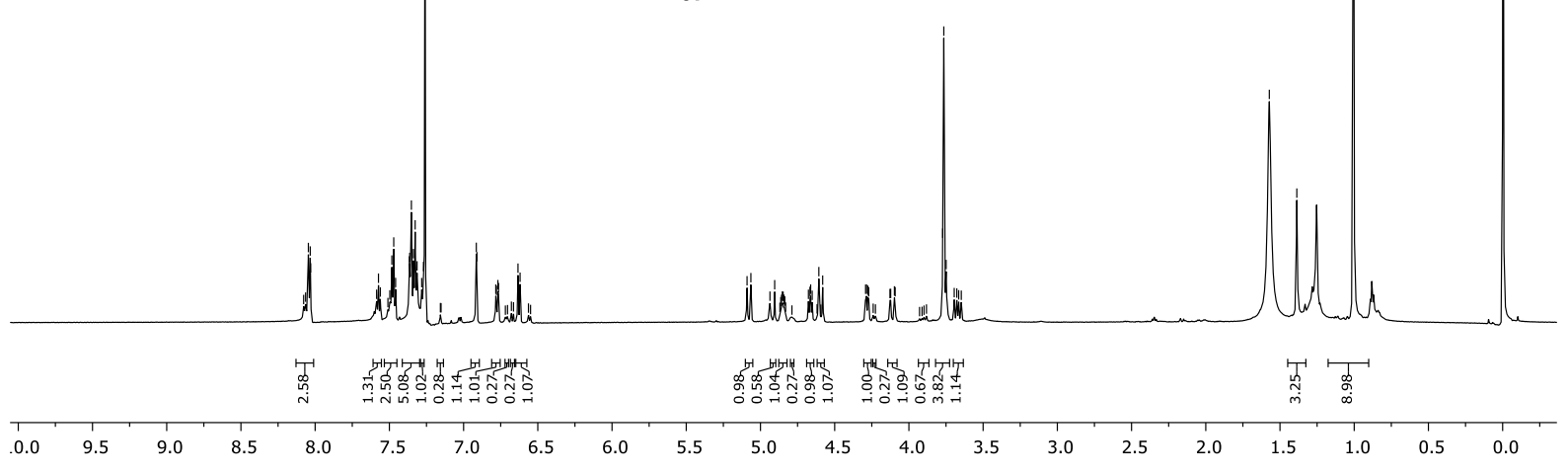

${ }^{13} \mathrm{C}\left\{{ }^{1} \mathrm{H}\right\}$ NMR of 31 (151 MHz, $\left.\mathrm{CDCl}_{3}\right)$



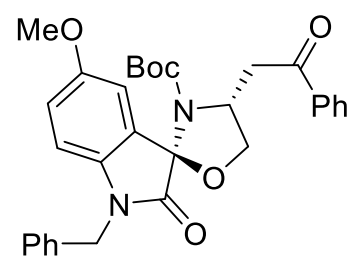

31

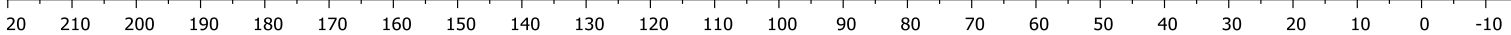


<smiles>Cc1ccc2c(c1)[C@@]1(C[C@@H](CC(=O)c3ccccc3)CO1)C(=O)N2Cc1ccccc1</smiles>

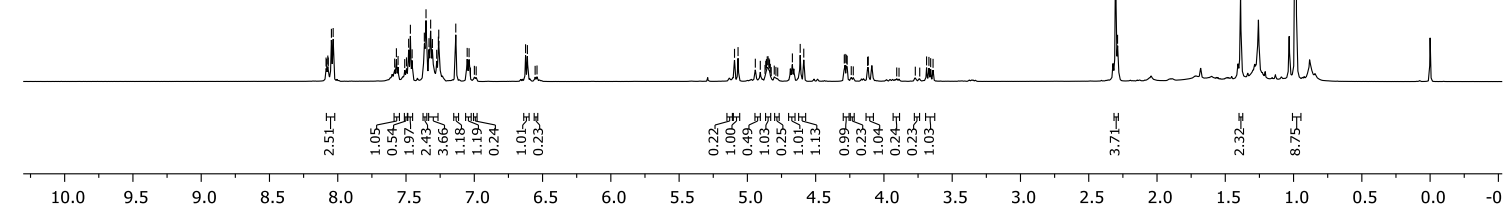

\section{${ }^{13} \mathrm{C}\left\{{ }^{1} \mathrm{H}\right\}$ NMR of $3 \mathrm{~m}\left(151 \mathrm{MHz}, \mathrm{CDCl}_{3}\right)$}

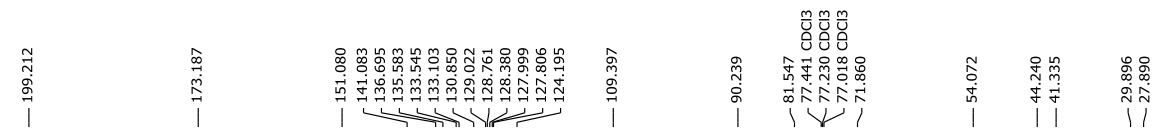
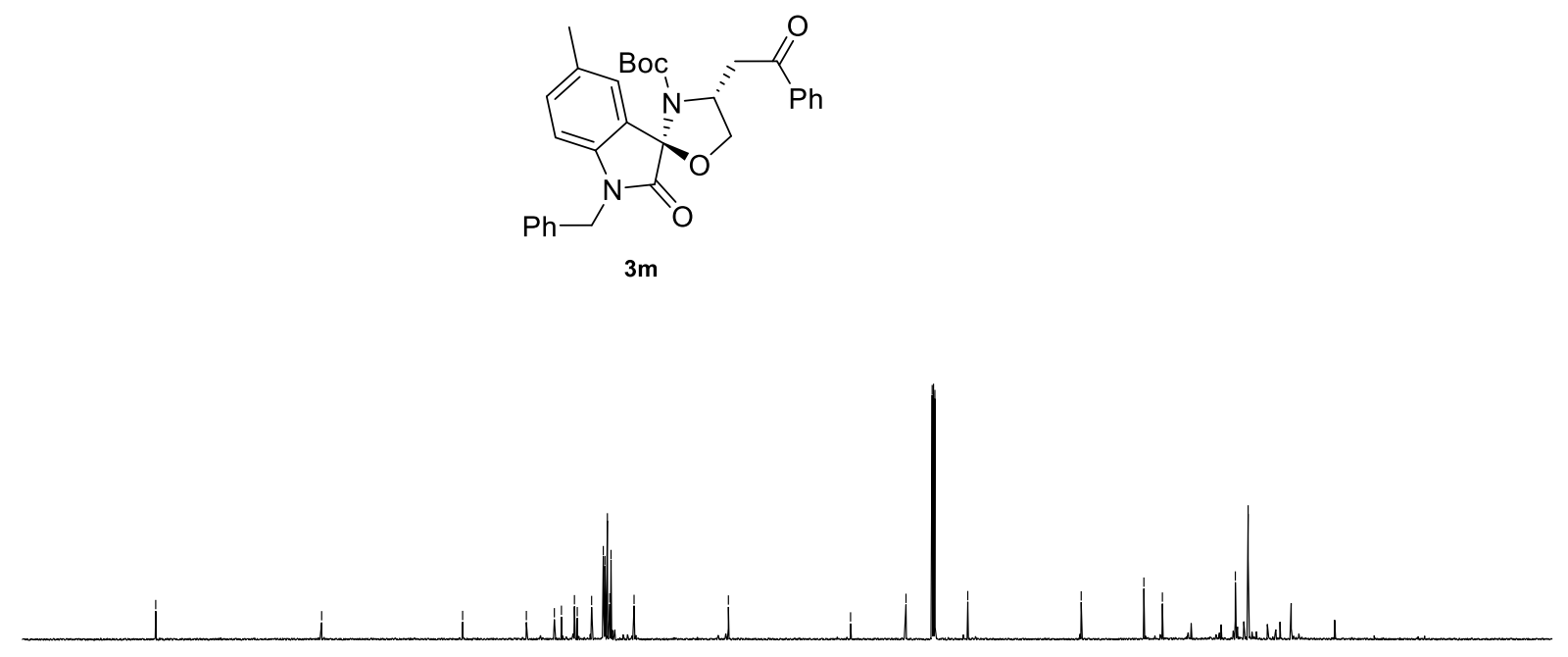

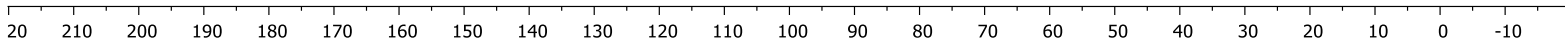


${ }^{1} \mathrm{H}$ NMR of $3 n\left(600 \mathrm{MHz}, \mathrm{CDCl}_{3}\right)$


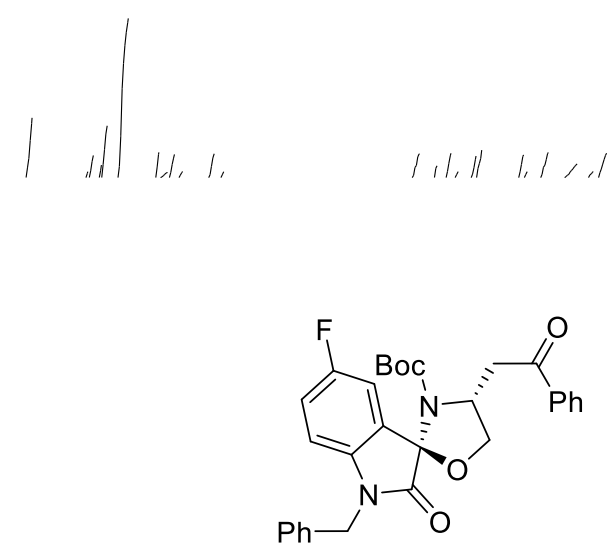

$3 n$

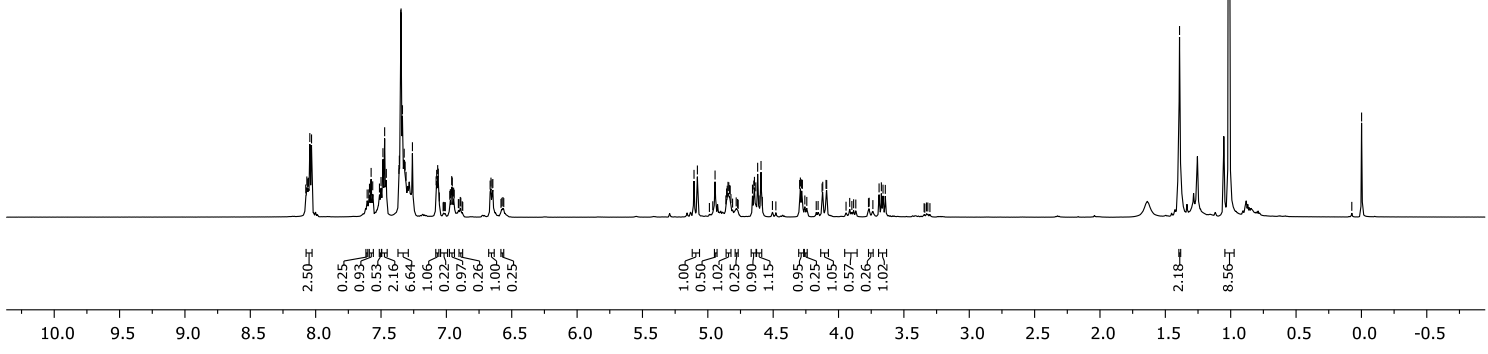

${ }^{13} \mathrm{C}\left\{{ }^{1} \mathrm{H}\right\}$ NMR of $3 n\left(151 \mathrm{MHz}, \mathrm{CDCl}_{3}\right)$

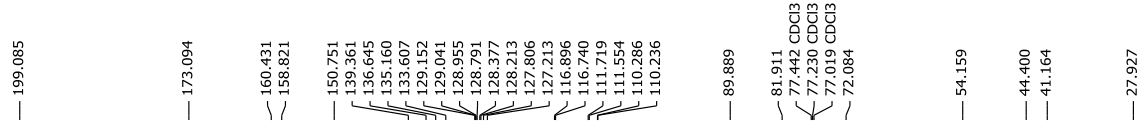

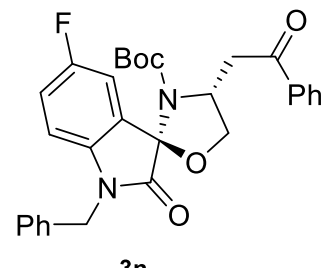

$3 n$



$\begin{array}{lllllllllllllllllllllllllll}20 & 210 & 200 & 190 & 180 & 170 & 160 & 150 & 140 & 130 & 120 & 110 & 100 & 90 & 80 & 70 & 60 & 50 & 40 & 30 & 20 & 10 & 0 & -10 & \end{array}$ 
${ }^{1} \mathrm{H}$ NMR of $30\left(400 \mathrm{MHz}, \mathrm{CDCl}_{3}\right)$

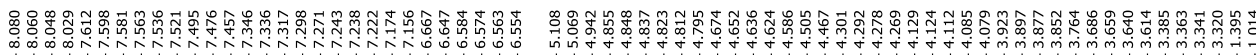
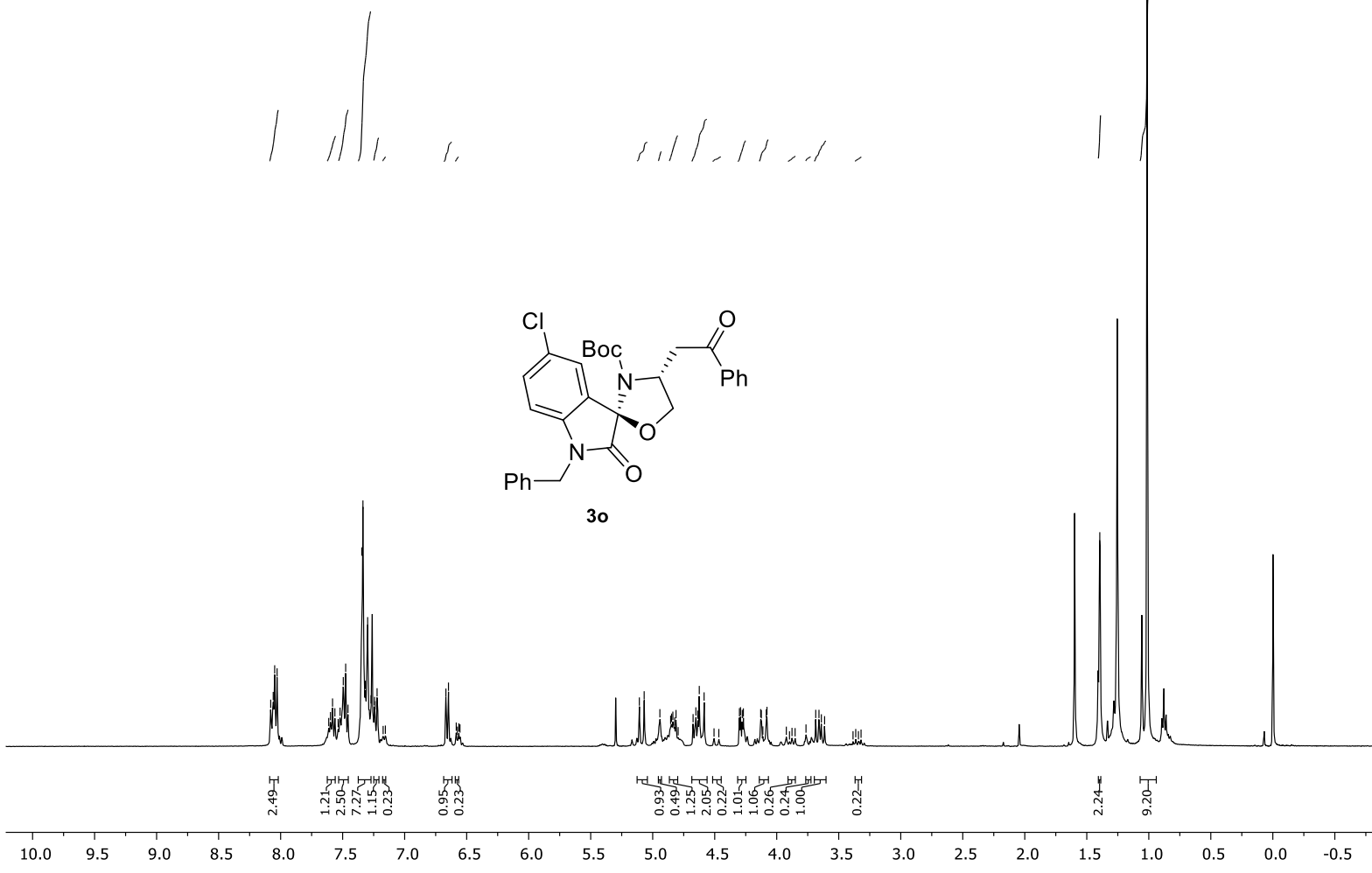

\section{${ }^{13} \mathrm{C}\left\{{ }^{1} \mathrm{H}\right\}$ NMR of $30\left(101 \mathrm{MHz}, \mathrm{CDCl}_{3}\right)$}



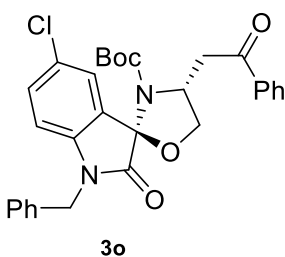

30

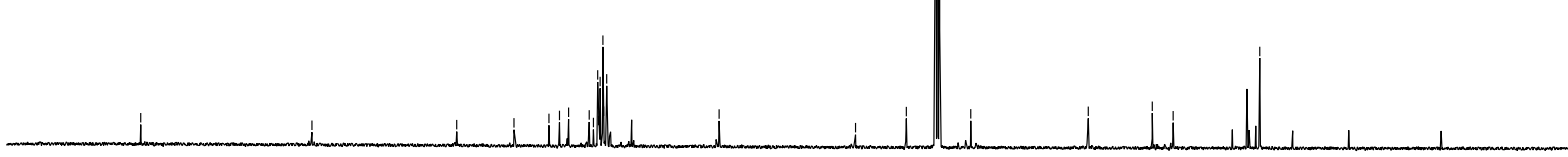

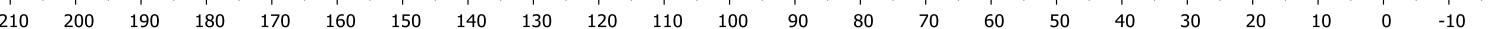


${ }^{1} \mathrm{H}$ NMR of 3p (400 $\left.\mathrm{MHz}, \mathrm{CDCl}_{3}\right)$





${ }^{13} \mathrm{C}\left\{{ }^{1} \mathrm{H}\right\}$ NMR of $3 p\left(101 \mathrm{MHz}, \mathrm{CDCl}_{3}\right)$
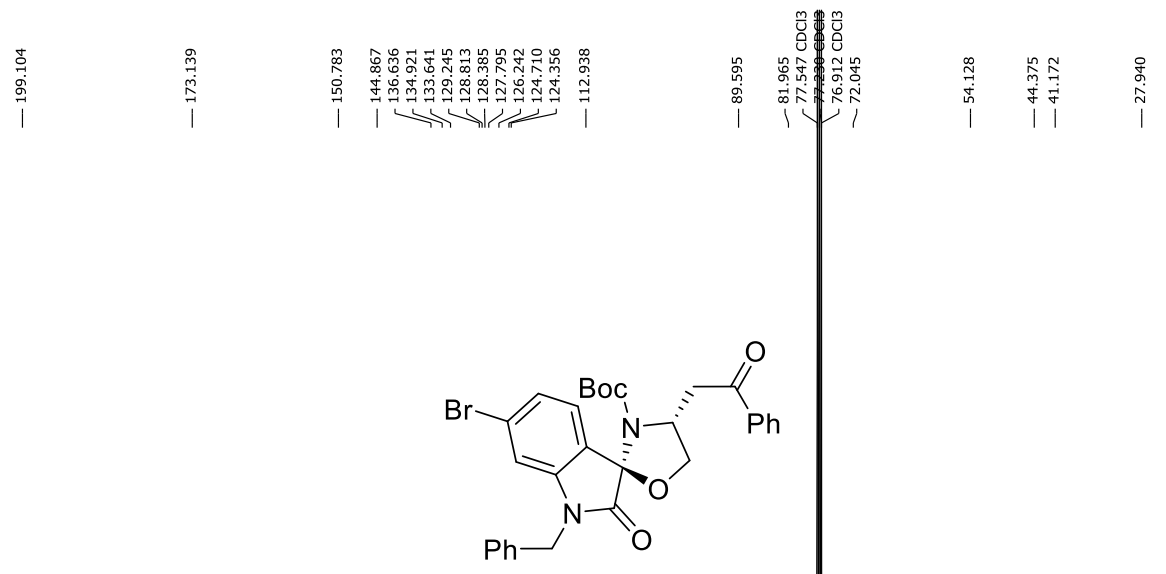

$3 p$

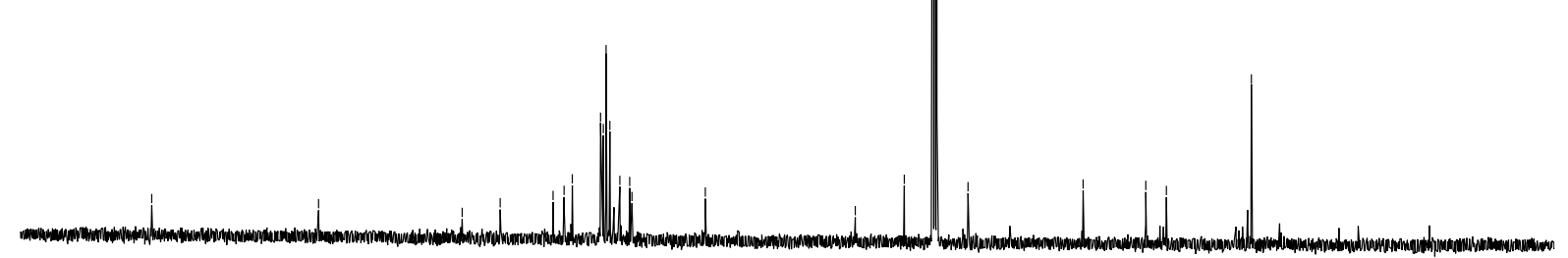

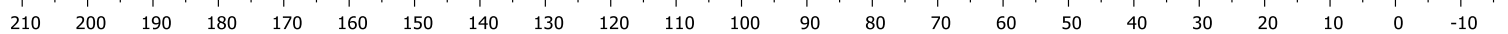


${ }^{1} \mathrm{H}$ NMR of 3q (400 MHz, $\left.\mathrm{CDCl}_{3}\right)$

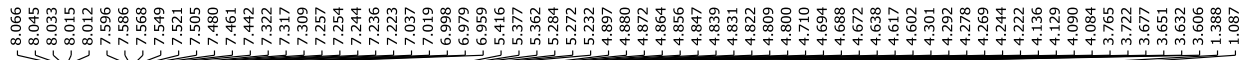

| I I

l,1, I, I, , , ,<smiles>CN1[C@H](CC(=O)c2ccccc2)CO[C@]12C(=O)N(Cc1ccccc1)c1c(Cl)cccc12</smiles>

$3 q$

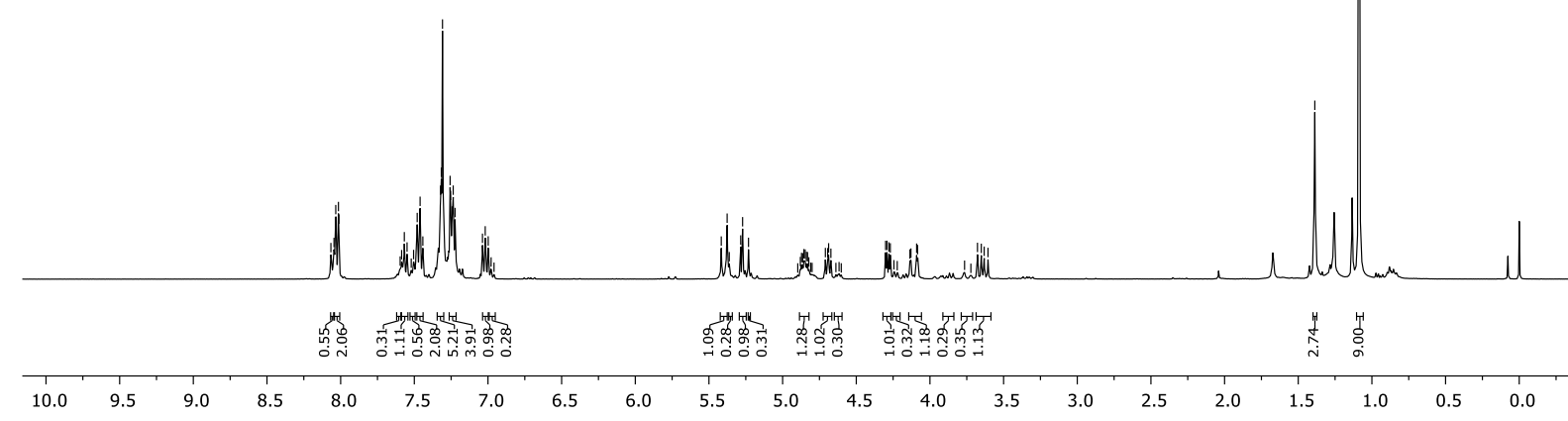

${ }^{13} \mathrm{C}\left\{{ }^{1} \mathrm{H}\right\}$ NMR of $3 q\left(101 \mathrm{MHz}, \mathrm{CDCl}_{3}\right)$



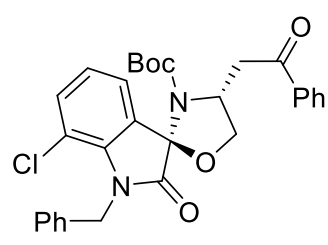

$3 \mathbf{q}$

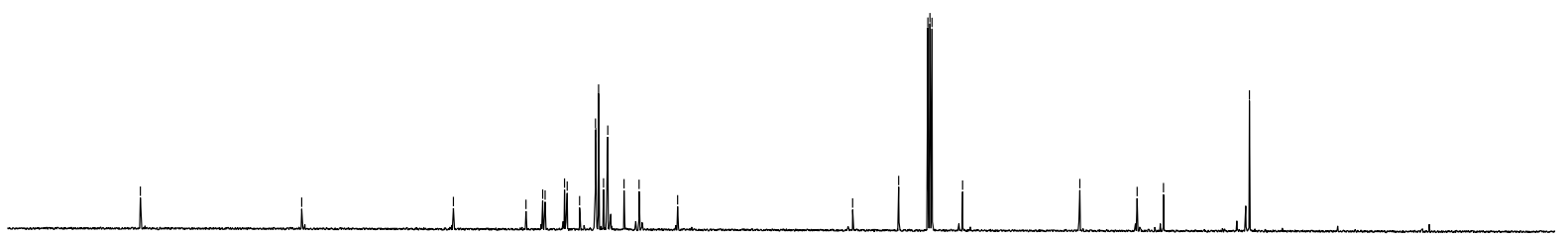

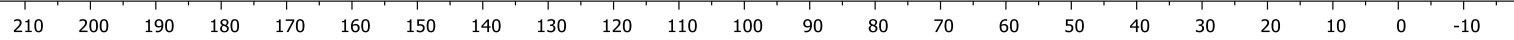


${ }^{1} \mathrm{H}$ NMR of $3 r\left(500 \mathrm{MHz}, \mathrm{CDCl}_{3}\right)$



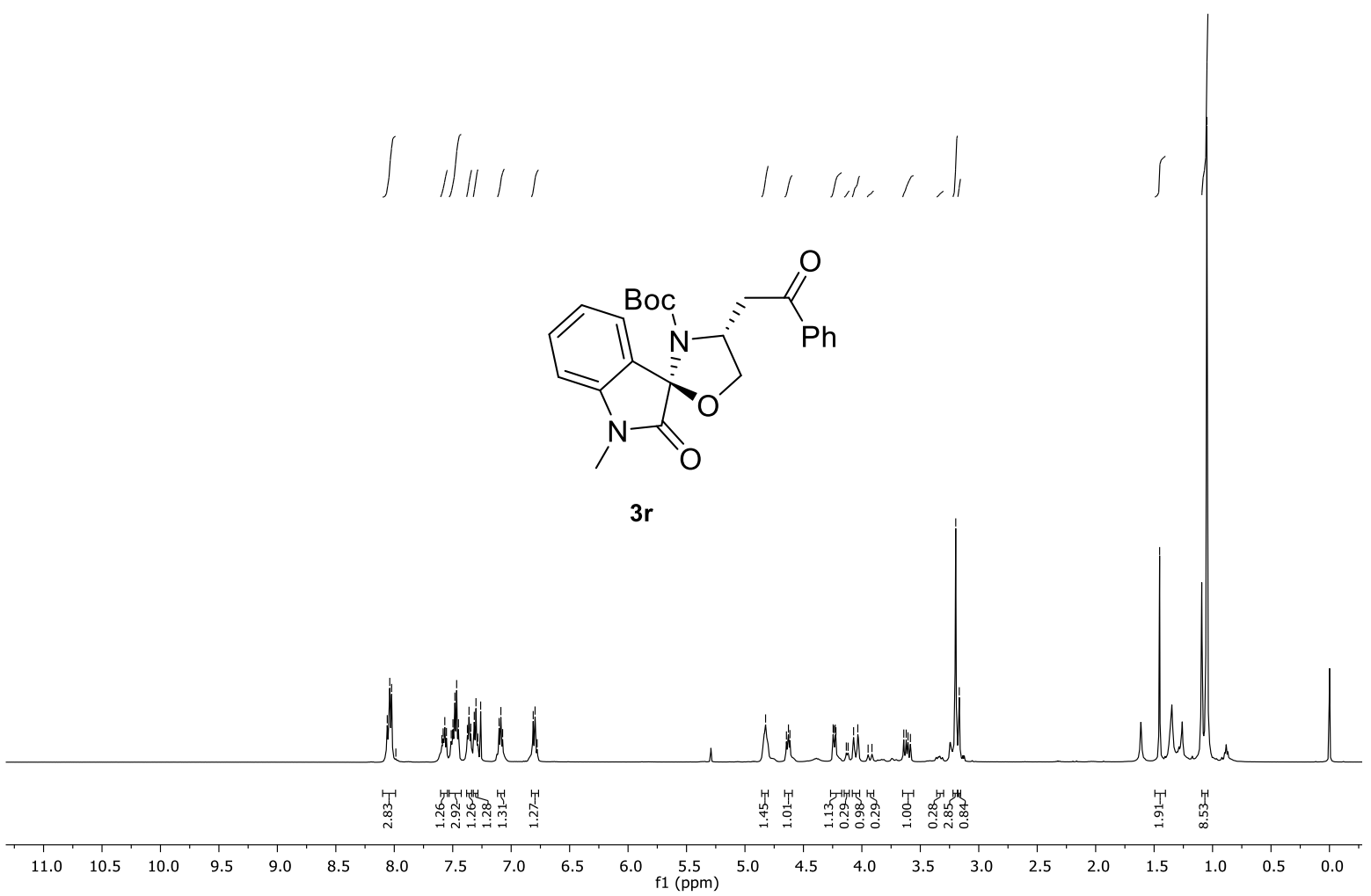

\section{${ }^{13} \mathrm{C}\left\{{ }^{1} \mathrm{H}\right\}$ NMR of $3 \mathrm{r}\left(151 \mathrm{MHz}, \mathrm{CDCl}_{3}\right)$}






${ }^{1} \mathrm{H}$ NMR of $3 \mathrm{~s}\left(400 \mathrm{MHz}, \mathrm{CDCl}_{3}\right)$

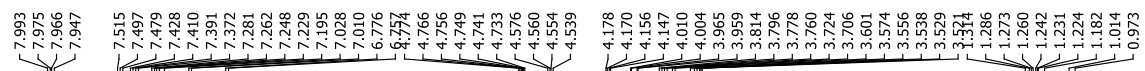

$\|, 1$

$1 / 1,1 / 1$,


3s

L Whold

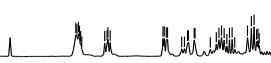





${ }^{13} \mathrm{C}\left\{{ }^{1} \mathrm{H}\right\}$ NMR of $3 \mathrm{~s}\left(151 \mathrm{MHz}, \mathrm{CDCl}_{3}\right)$

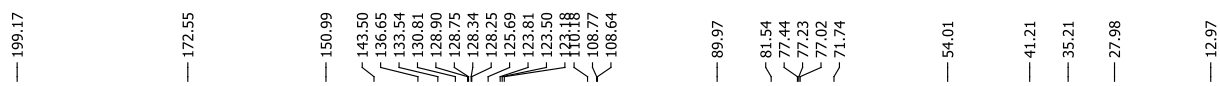

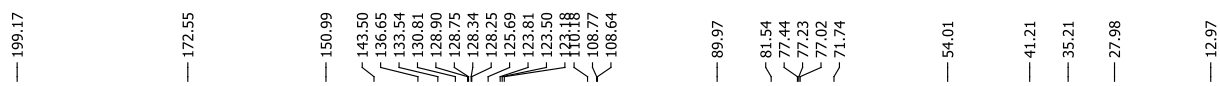

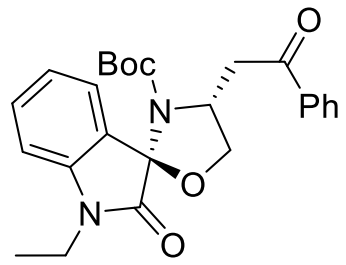

3s



$\begin{array}{llllllllllllllllllllllllllllll}220 & 210 & 200 & 190 & 180 & 170 & 160 & 150 & 140 & 130 & 120 & 110 & 100 & 90 & 80 & 70 & 60 & 50 & 40 & 30 & 20 & 10 & 0 & -10\end{array}$ 
<smiles>CCCN1C(=O)C2(OC[C@H](CC(=O)c3ccccc3)N2C(=O)OCc2ccccc2)c2ccccc21</smiles>

3t

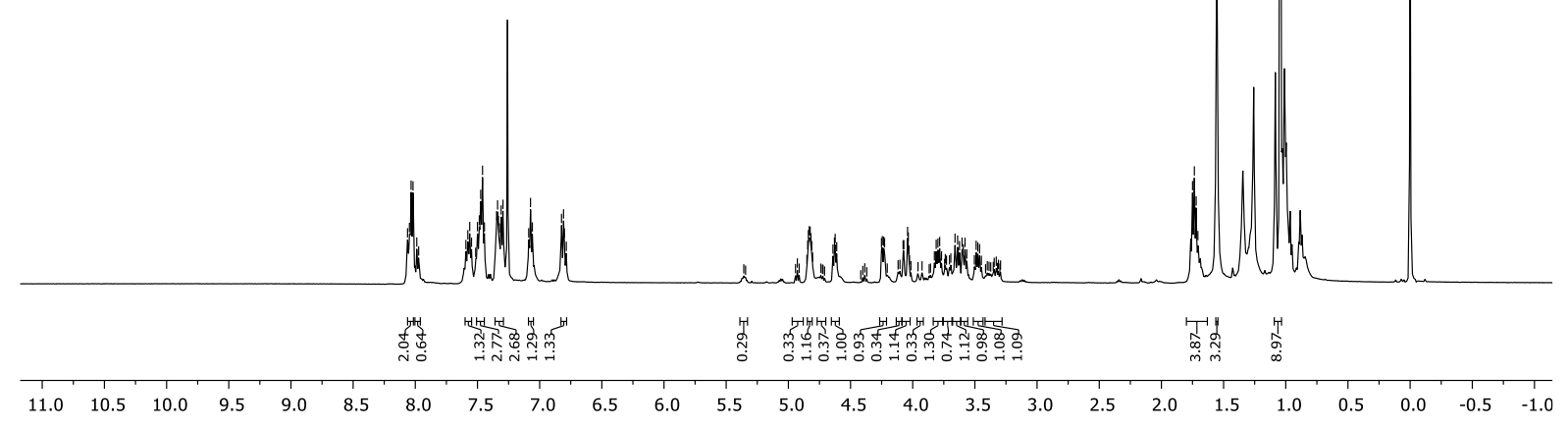

\section{${ }^{13} \mathrm{C}\left\{{ }^{1} \mathrm{H}\right\}$ NMR of $3 \mathrm{t}\left(151 \mathrm{MHz}, \mathrm{CDCl}_{3}\right)$}

(



$3 \mathbf{t}$

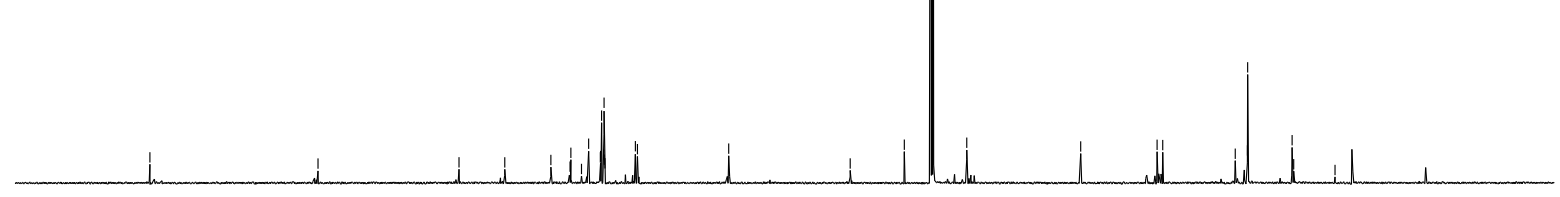

$\begin{array}{llllllllllll}220 & 210 & 200 & 190 & 180 & 170 & 160 & 150 & 140 & 130 & 120 & 110 \\ \mathrm{f} 1(\mathrm{ppm}) & 100 & 90\end{array}$ 



4

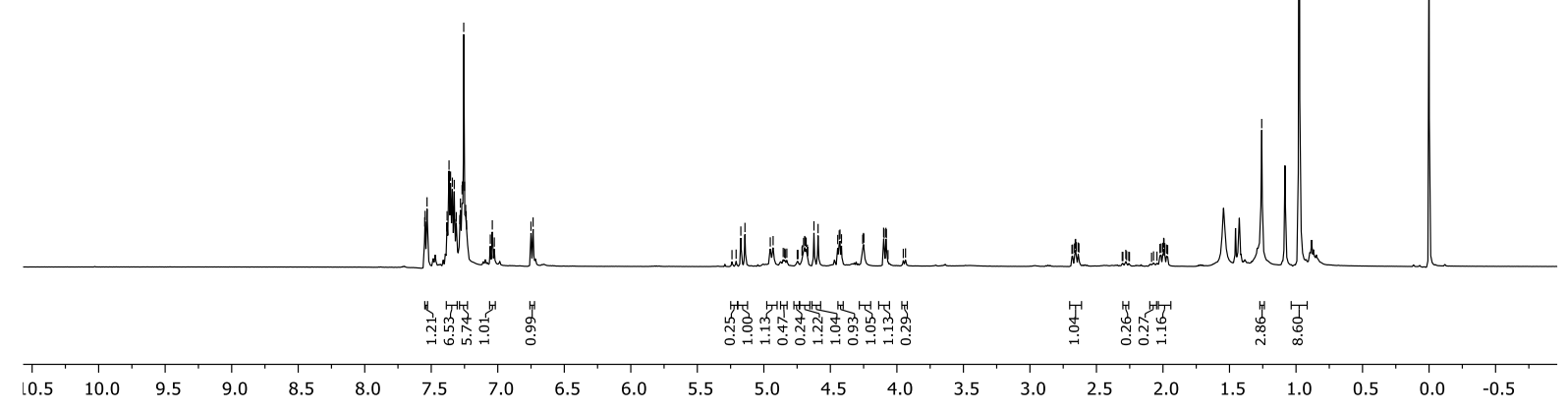

${ }^{13} \mathrm{C}\left\{{ }^{1} \mathrm{H}\right\}$ NMR of $4\left(151 \mathrm{MHz}, \mathrm{CDCl}_{3}\right)$



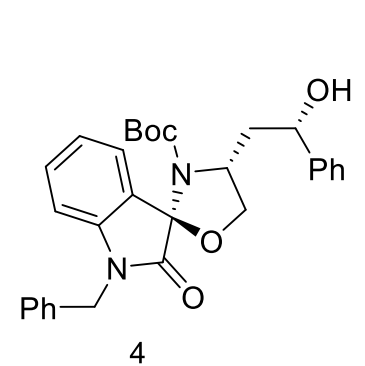

4

$\begin{array}{lllllllllllll}220 & 210 & 200 & 190 & 180 & 170 & 160 & 150 & 140 & 130 & 120 & 110 \underset{\mathrm{f} 1(\mathrm{ppm})}{100} & 90\end{array}$ 




NOESY spectra of compound 4 exhibiting the strong interaction between the protons (marked as $\mathrm{Ha}$ and $\mathrm{Hb}$ ), so we can conclude that both the $\mathrm{Ha}$ and $\mathrm{Hb}$ are in the same plane. 
${ }^{1} \mathrm{H}$ NMR of $5\left(500 \mathrm{MHz}, \mathrm{CDCl}_{3}\right)$



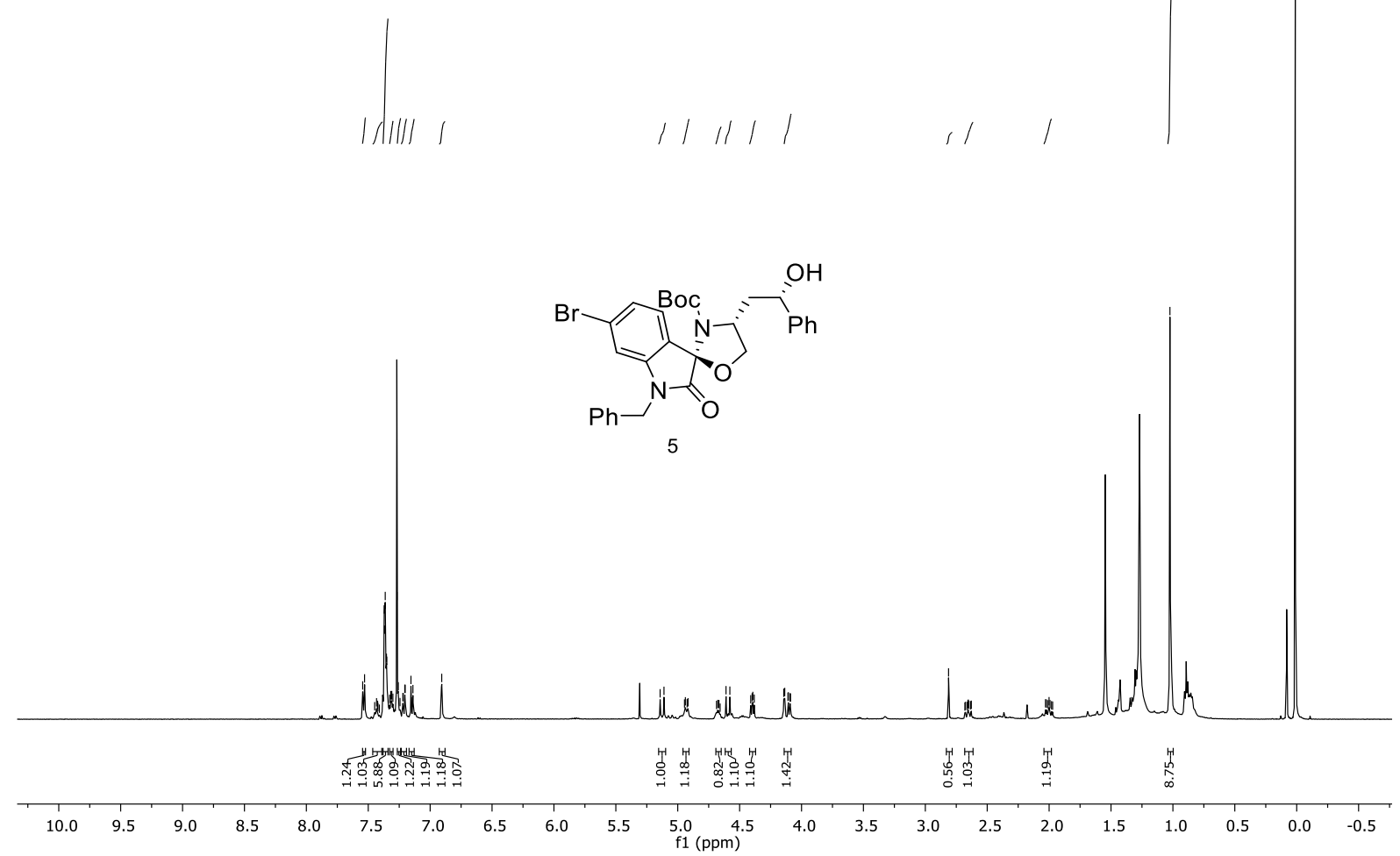

${ }^{13} \mathrm{C}\left\{{ }^{1} \mathrm{H}\right\}$ NMR of $5\left(126 \mathrm{MHz}, \mathrm{CDCl}_{3}\right)$



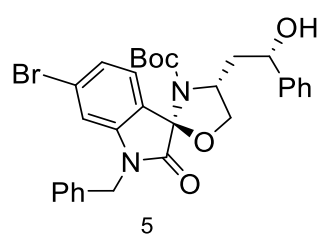

$\begin{array}{lllllllllllllllllllllll}210 & 200 & 190 & 180 & 170 & 160 & 150 & 140 & 130 & 120 & 110 & 100 & 90 & 80 & 70 & 60 & 50 & 40 & 30 & 20 & 10 & 0 & -10\end{array}$ 
${ }^{1} \mathrm{H}$ NMR of $7\left(600 \mathrm{MHz}, \mathrm{CDCl}_{3}\right)$

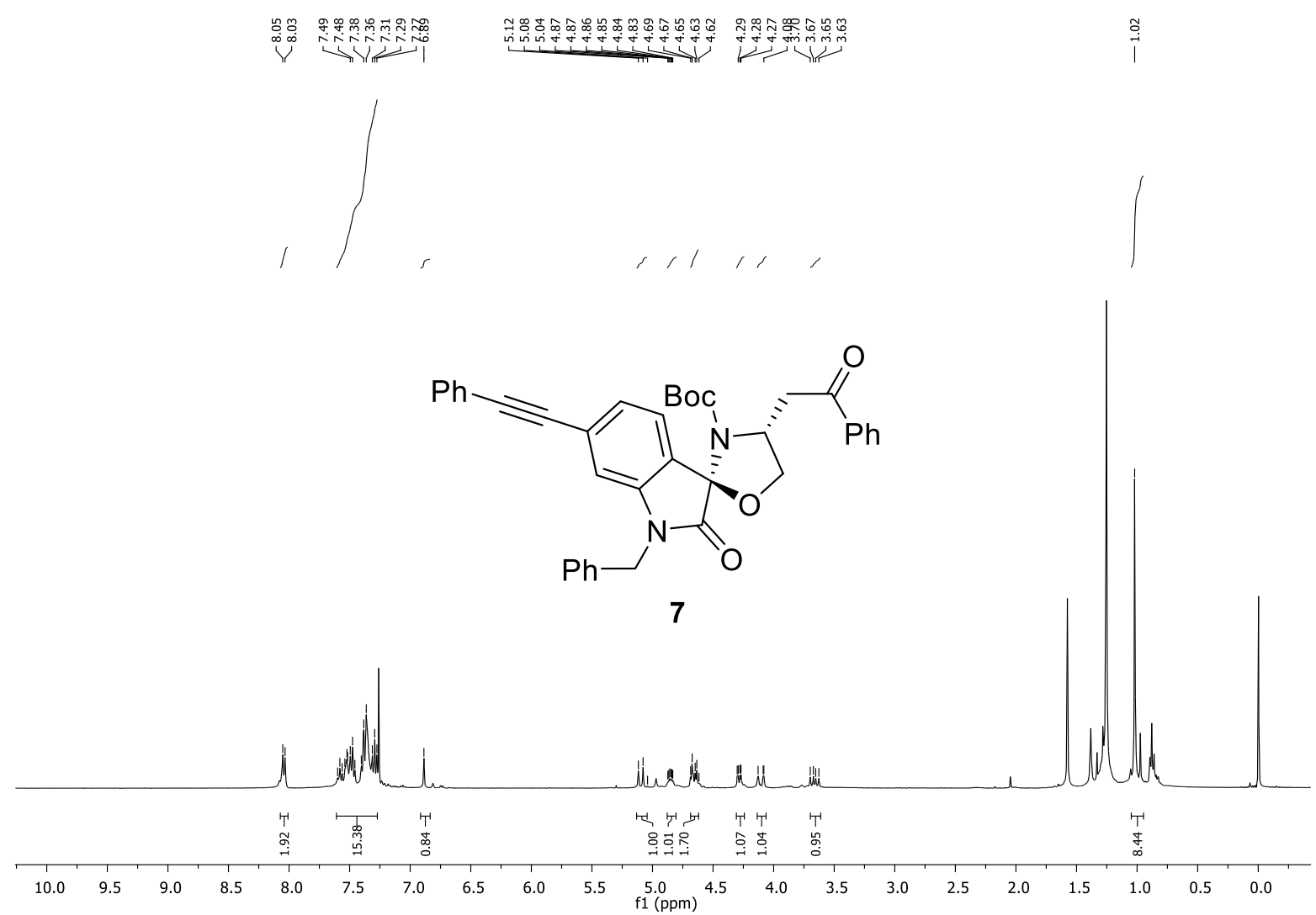

${ }^{13} \mathrm{C}\left\{{ }^{1} \mathrm{H}\right\}$ NMR of $7\left(101 \mathrm{MHz}, \mathrm{CDCl}_{3}\right)$

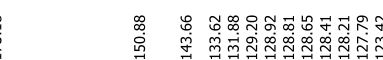

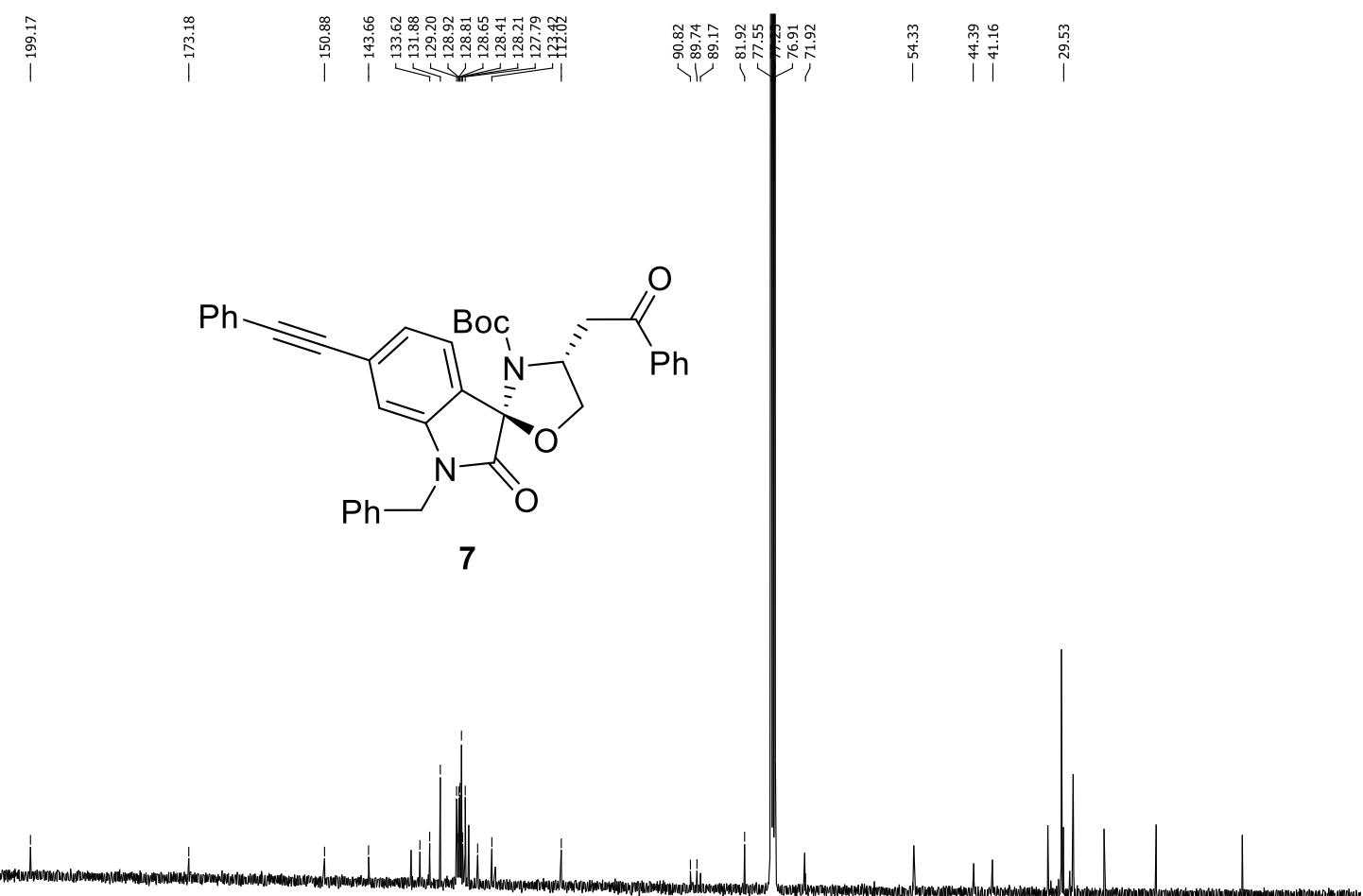

$\begin{array}{lllllllllllllllllllllll}210 & 200 & 190 & 180 & 170 & 160 & 150 & 140 & 130 & 120 & 110 & \begin{array}{c}100 \\ \mathrm{f} 1(\mathrm{ppm})\end{array} & 90 & 80 & 70 & 60 & 50 & 40 & 30 & 20 & 10 & 0 & -10\end{array}$ 


\section{HPLC spectra of the products:}
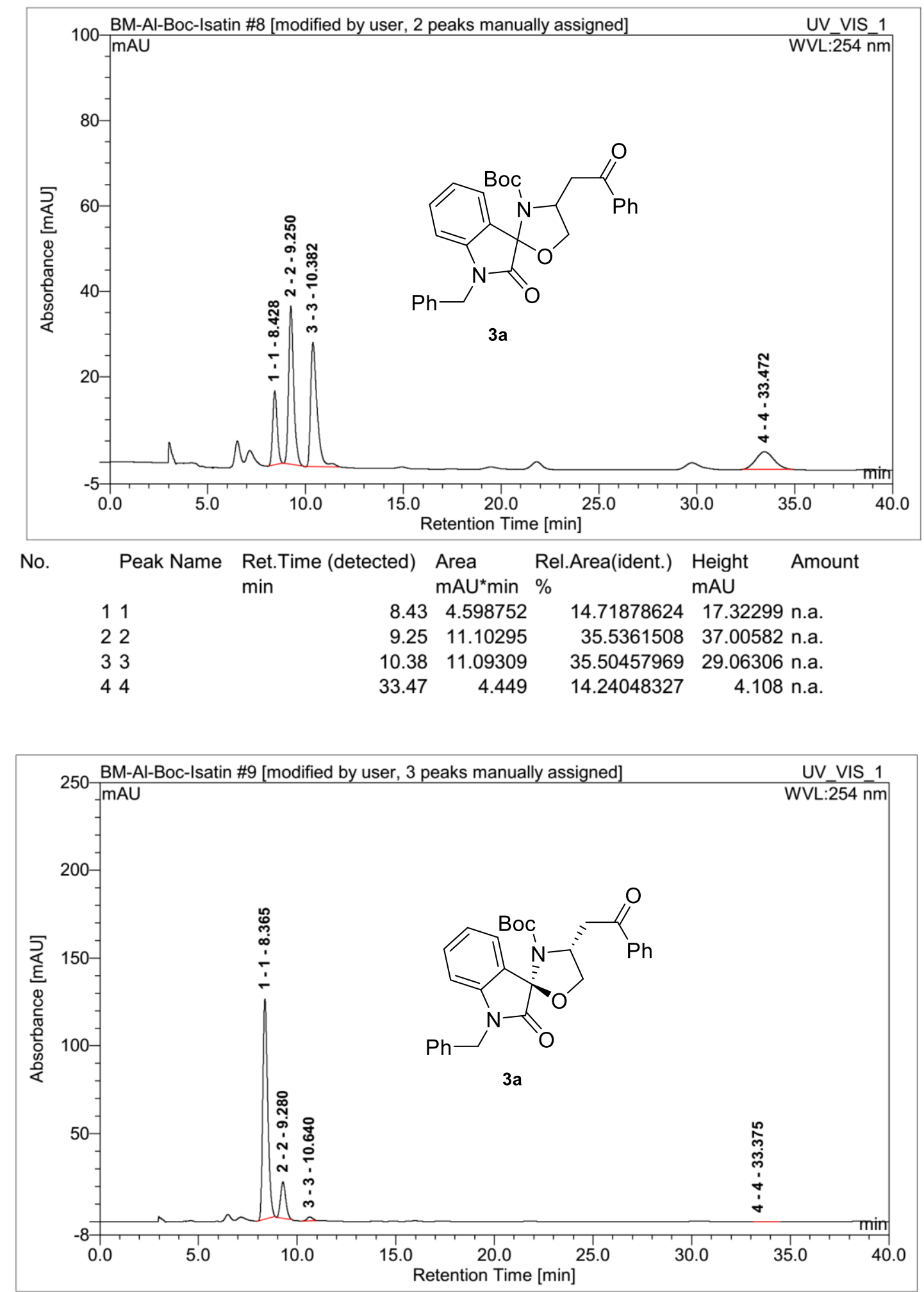

\begin{tabular}{|c|c|c|c|c|c|c|}
\hline No. & Peak Name & $\begin{array}{l}\text { Ret.Time (detected) } \\
\min \end{array}$ & $\begin{array}{l}\text { Area } \\
\mathrm{mAU}{ }^{*} \min \end{array}$ & $\begin{array}{l}\text { Rel.Area(ident.) } \\
\%\end{array}$ & $\begin{array}{l}\text { Height } \\
\text { mAU }\end{array}$ & Amount \\
\hline & 11 & 8.37 & 36.60123 & 83.77327251 & 125.3329 & n.a. \\
\hline & 22 & 9.28 & 6.370305 & 14.58041763 & 20.74822 & n.a. \\
\hline & 33 & 10.64 & 0.702765 & 1.608494905 & 2.18771 & n.a. \\
\hline & 44 & 33.38 & 0.017 & 0.03781495844 & 0.027 & n.a. \\
\hline
\end{tabular}




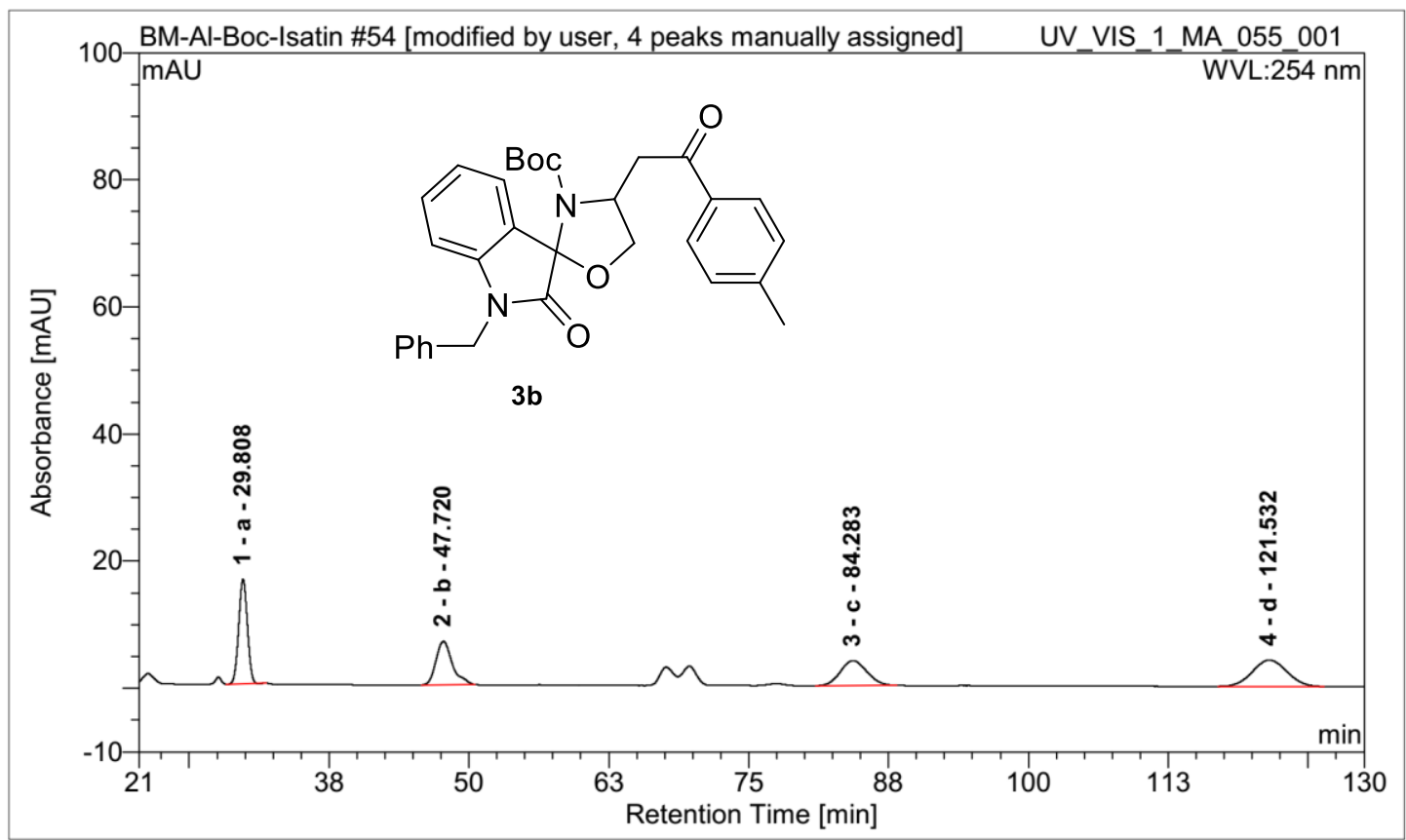


$1 \mathrm{a}$
$2 \mathrm{~b}$
29.8114 .76184
28.4340416816 .47746 n.a.
$3 \mathrm{c}$
$\begin{array}{ll}47.72 & 11.58023\end{array}$
22.30566671
6.82911 n.a.
$84.28 \quad 10.7867$
20.77719237
3.91198 n.a.
$4 \mathrm{~d}$
$121.53 \quad 14.787$
28.48309924
4.152 n.a.

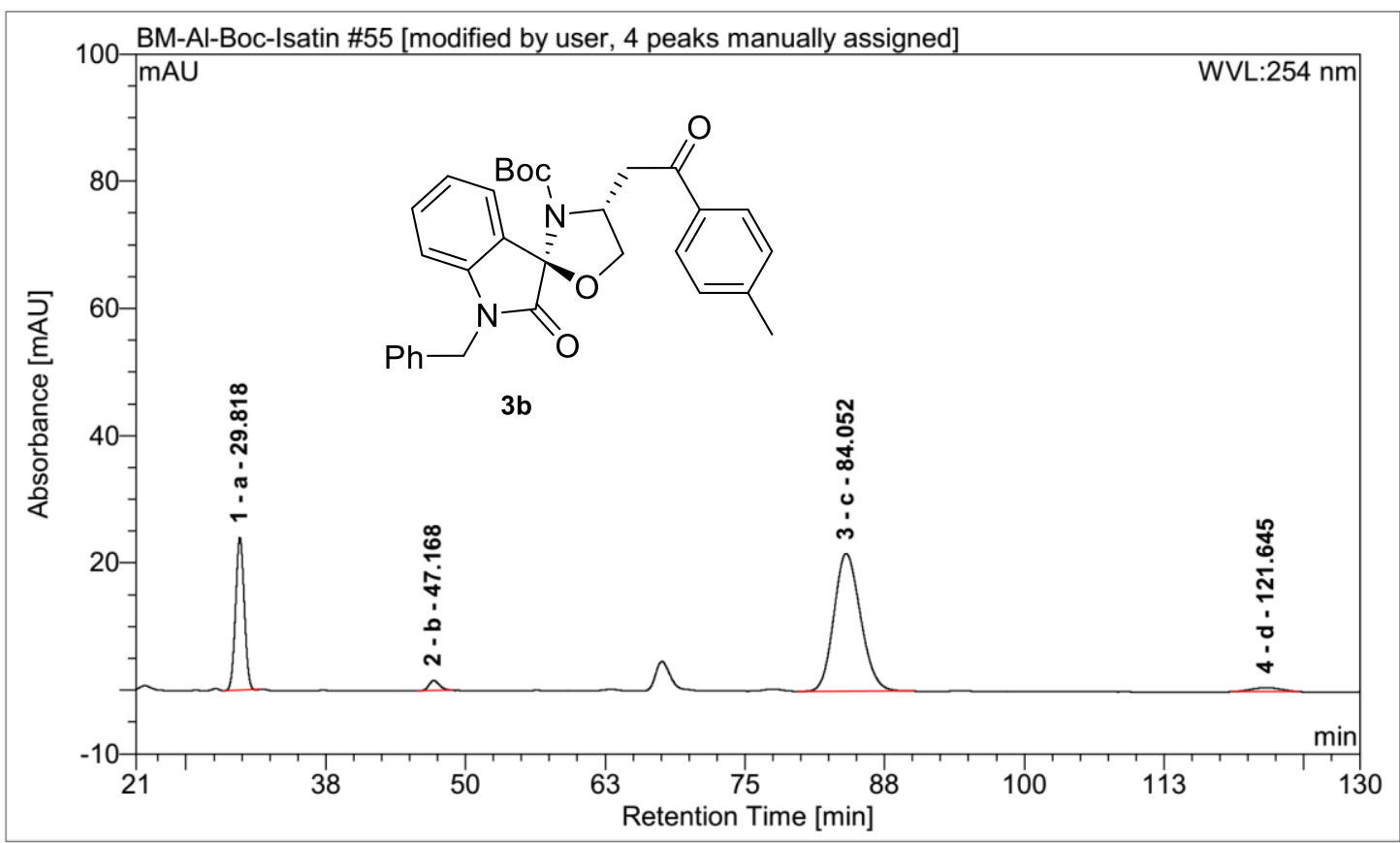



$\begin{array}{lrrrr}1 \mathrm{a} & 29.82 & 21.68747 & 25.4007429 & 24.03502 \text { n.a. } \\ 2 \mathrm{~b} & 47.17 & 1.777982 & 2.082403549 & 1.59535 \text { n.a. } \\ 3 \mathrm{c} & 84.05 & 60.02303 & 70.30001583 & 21.63926 \text { n.a. } \\ 4 \mathrm{~d} & 121.65 & 1.893 & 2.216837721 & 0.609 \text { n.a. }\end{array}$




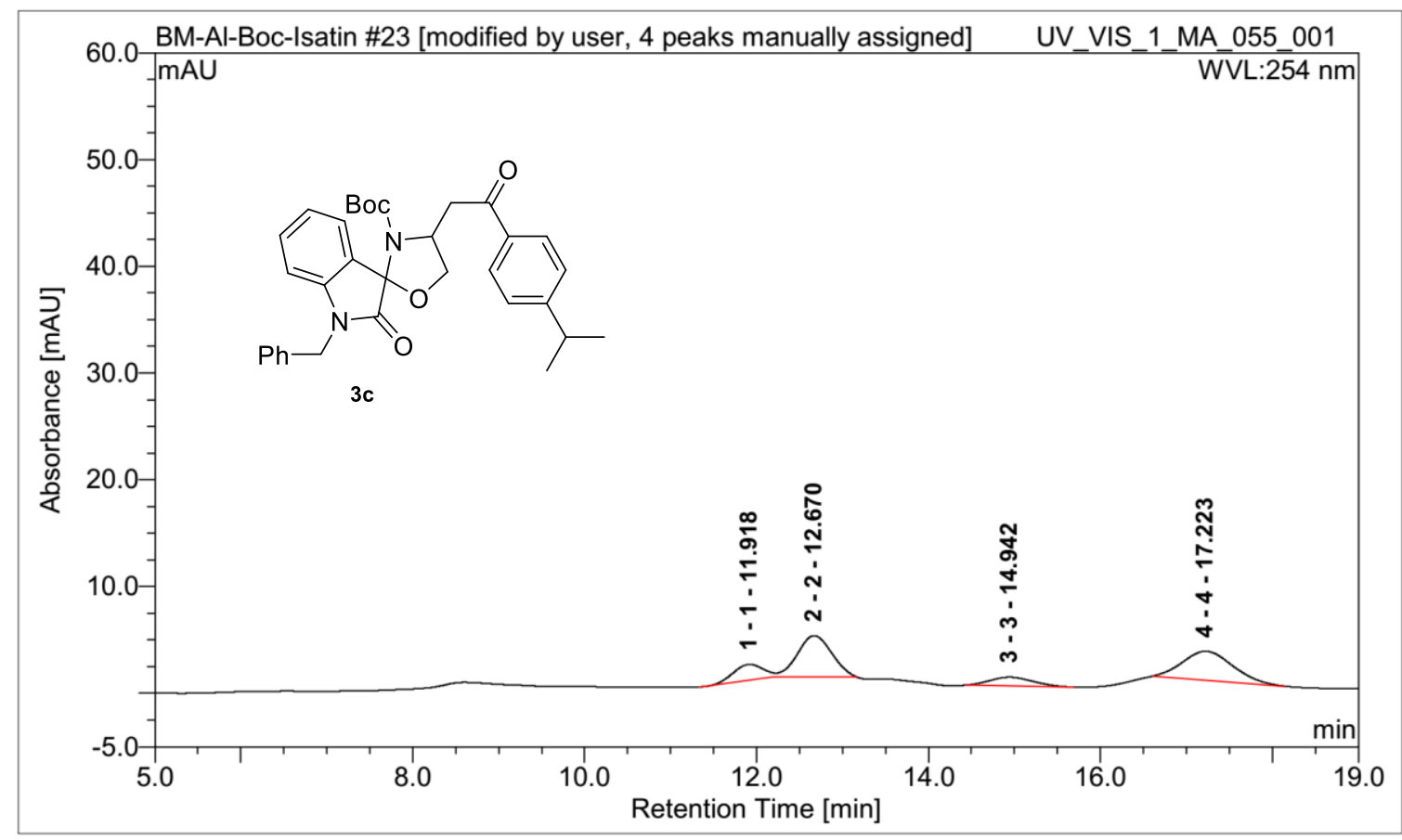

No.

Peak Name Ret.Time (detected) $\min$

Area $\mathrm{mAU} * \min \%$

11

22

33

44
$11.92 \quad 0.577682$

$12.67 \quad 1.803854$

$14.94 \quad 0.483319$

$17.22 \quad 1.830$
Rel.Area(ident.) Height Amount mAU

$12.30472433 \quad 1.45878$ n.a.

$38.42243386 \quad 3.86205$ n.a.

$10.29478549 \quad 0.82679$ n.a.

$38.97805632 \quad 2.700$ n.a.

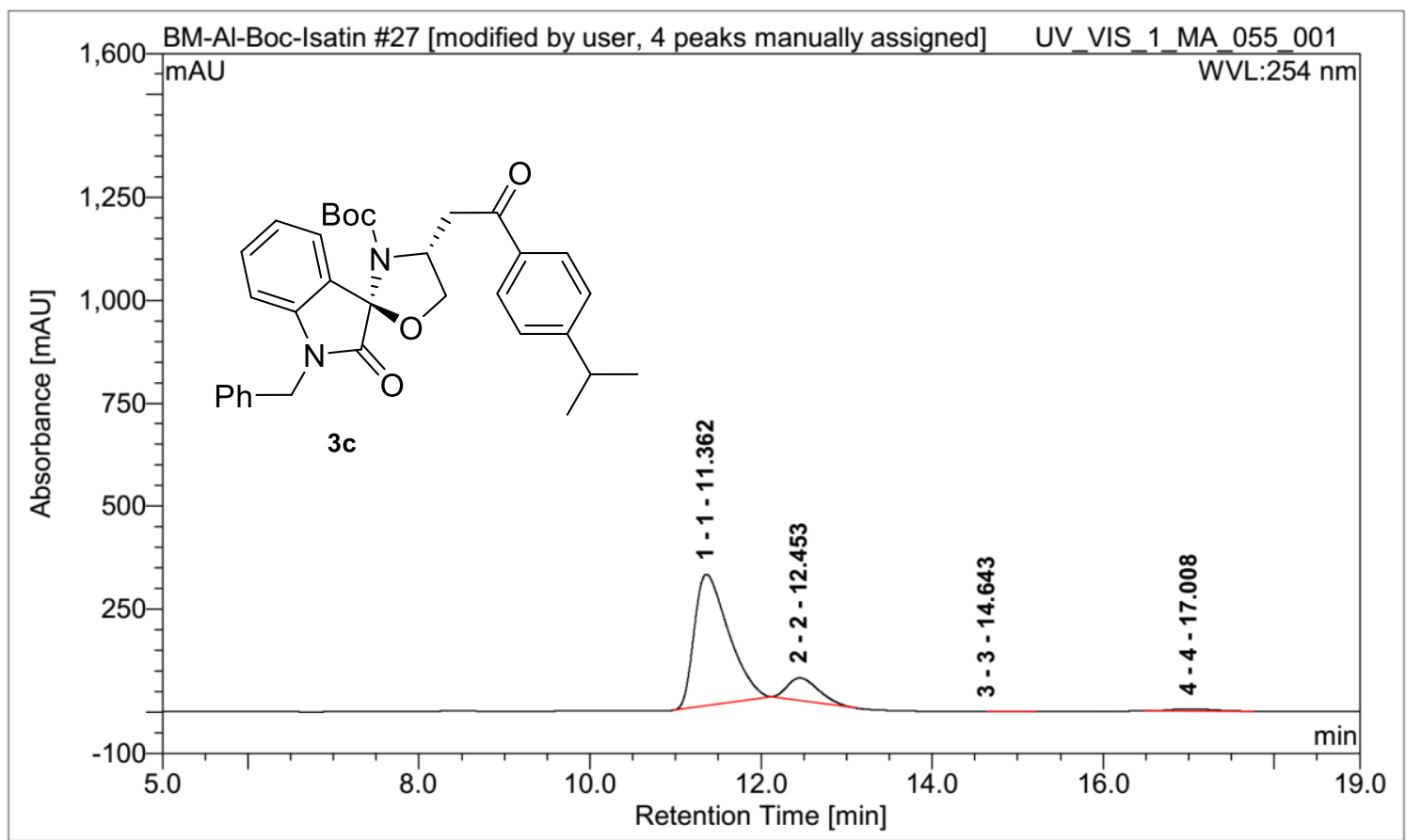

No.

$$
\text { Peak Name }
$$

11

22

33

44 Ret.
min
Area $\mathrm{mAU}{ }^{*} \min \%$

$11.36 \quad 148.4399$

$12.45 \quad 23.35628$

$14.64 \quad 0.033423$

$17.01 \quad 3.085$
Rel.Area(ident.) Height Amount mAU

$\begin{array}{ll}84.86434896 & 319.3825 \\ 13.35298715 & 54.89481 \text { n.a. }\end{array}$

0.019108303310 .00112 n.a.

$1.763555582 \quad 5.393$ n.a. 


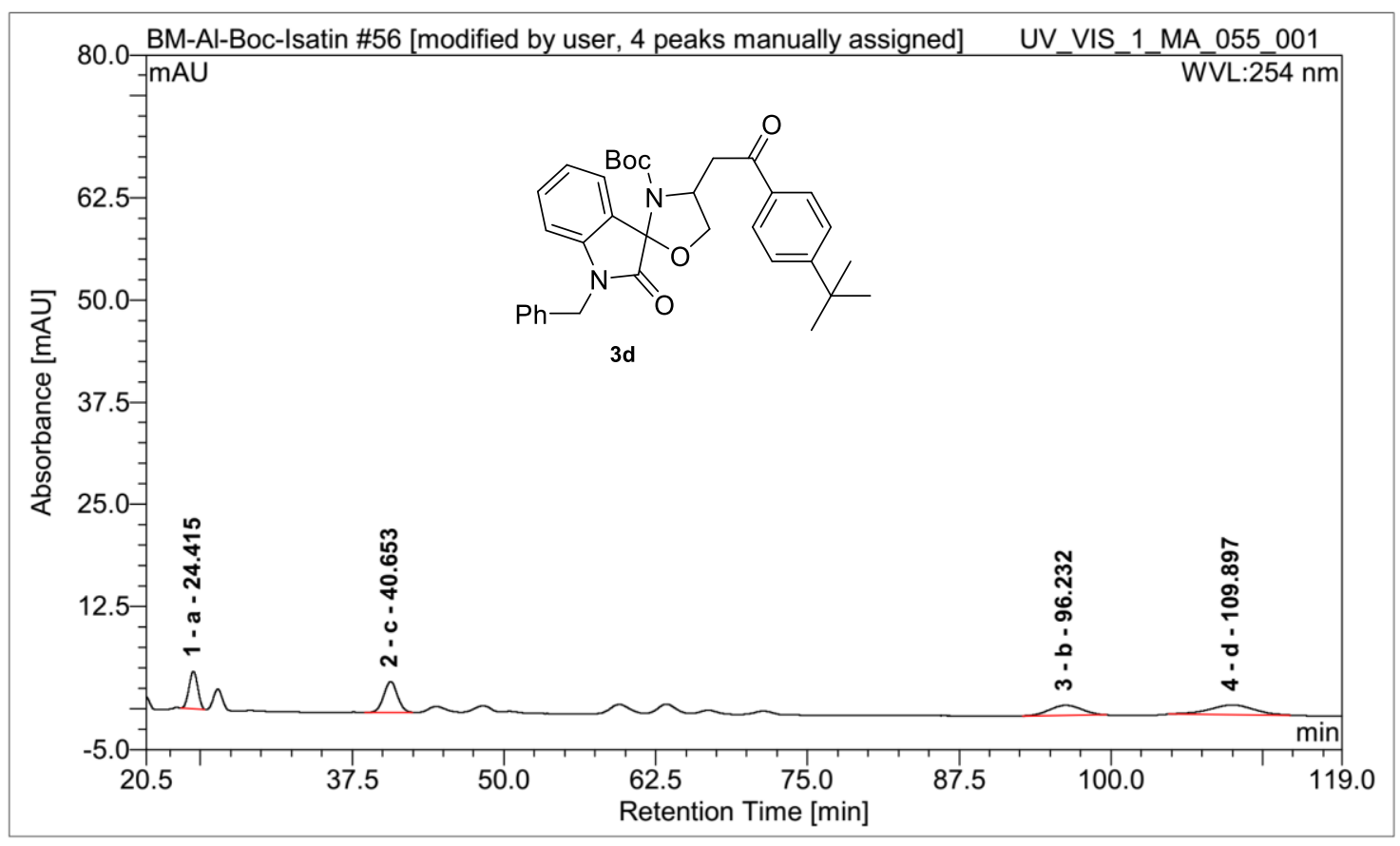

No.

\section{Peak Name}

$1 \mathrm{a}$
$2 \mathrm{c}$
$3 \mathrm{~b}$

Ret.Ti
$\min$



$\begin{array}{lll}\text { detected) } & \text { Area } & \text { Rel.A } \\ & \text { mAU*min \% } \\ 24.42 & 3.822713\end{array}$

$\begin{array}{ll}24.42 & 3.822713 \\ 40.65 & 4.949027 \\ 96.23 & 3.809804\end{array}$

$\begin{array}{rr}96.23 & 3.809804 \\ 109.90 & 5.010\end{array}$
Rel.Area(ident.) Height Amount mAU

21.730167194 .60425 n.a.

$28.13268394 \quad 3.77687$ n.a.

$21.65678831 \quad 1.23486$ n.a.

$28.48036056 \quad 1.180$ n.a.



No.

\section{$\begin{aligned} \text { Peak Name } & \text { Ret.Tim } \\ & \text { min }\end{aligned}$}

1 a

$2 \mathrm{~b}$

$3 \mathrm{c}$

$4 \mathrm{~d}$
Area $\mathrm{mAU}{ }^{*} \min \%$

$\begin{array}{rr}24.31 & 16.365 \\ 40.54 & 0.206332 \\ 95.89 & 2.108121\end{array}$

$106.13 \quad 70.811$
Rel.Area(ident.) Height Amount mAU $18.2868487 \quad 19.30699$ n.a. 0.23056283230 .17038 n.a. $2.355692079 \quad 0.6369$ n.a. $79.12689639 \quad 14.223$ n.a. 


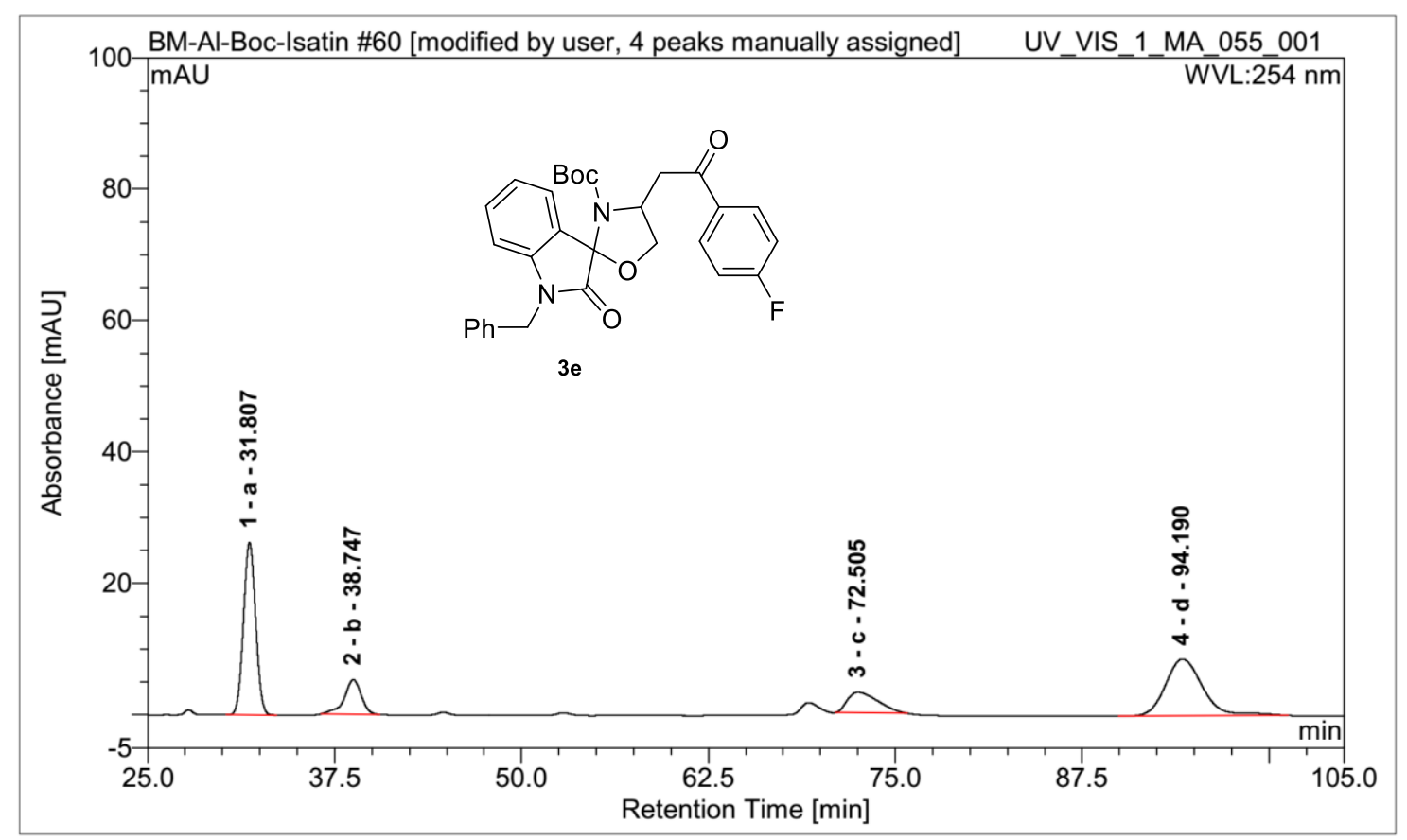

No. Peak Name Ret.Time (detected) Area Rel.Area(ident.) Height Amount $\min \quad \mathrm{mAU}^{*} \min \% \quad \mathrm{mAU}$

$\begin{array}{rrrrr}1 \mathrm{a} & 31.81 & 24.67472 & 38.43889059 & 26.23816 \text { n.a. } \\ 2 \mathrm{~b} & 38.75 & 6.958204 & 10.83966271 & 5.28147 \text { n.a. } \\ 3 \mathrm{c} & 72.51 & 7.544021 & 11.75226343 & 3.14492 \text { n.a. } \\ 4 \mathrm{~d} & 94.19 & 25.015 & 38.96918326 & 8.657 \text { n.a. }\end{array}$

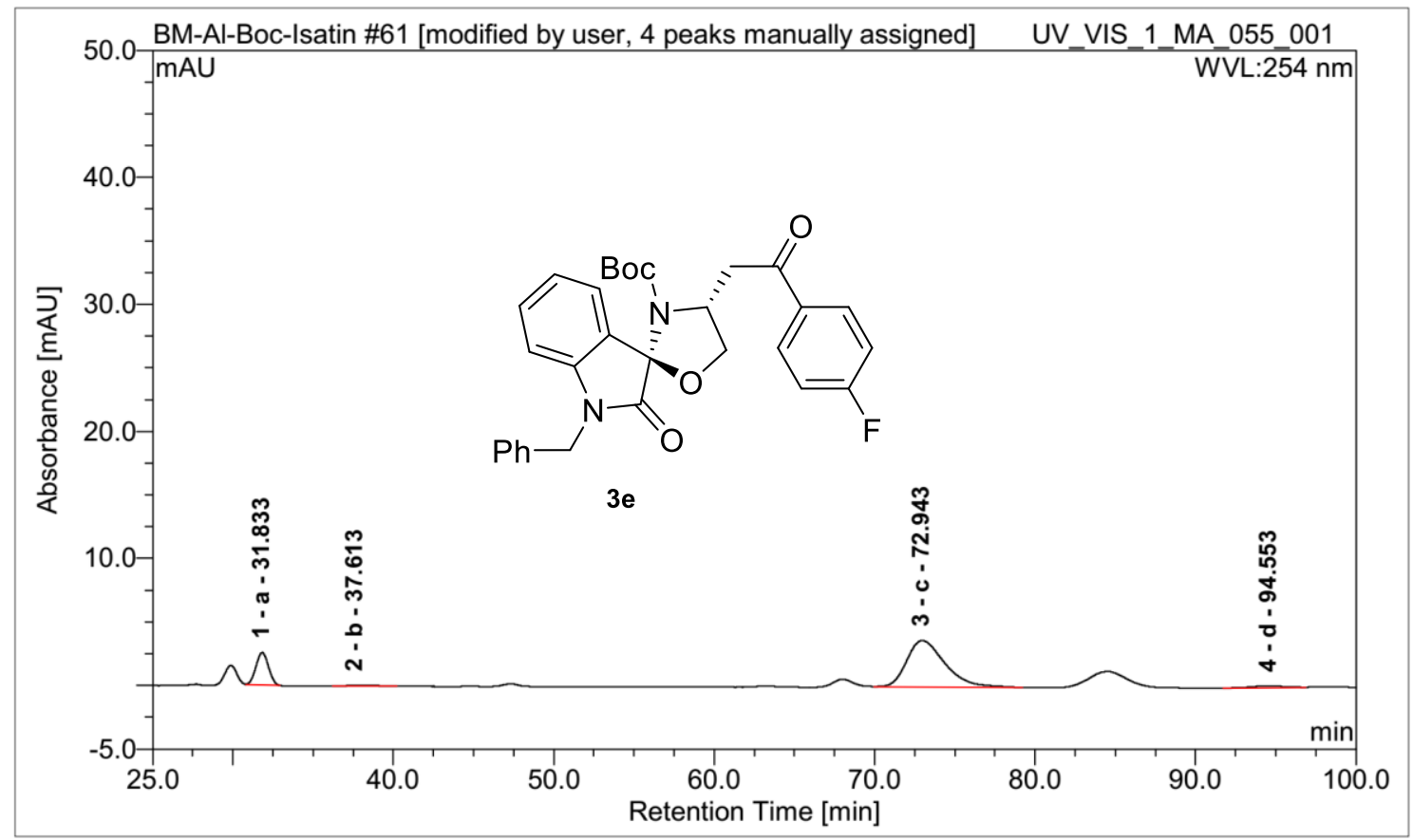

No.

\begin{tabular}{lllrrr} 
Peak Name & $\begin{array}{l}\text { Ret.Time (detected) } \\
\text { min }\end{array}$ & $\begin{array}{l}\text { Area } \\
m A U^{*} \min \%\end{array}$ & \multicolumn{2}{l}{$\begin{array}{l}\text { Rel.Area(ident.) } \\
\text { Height }\end{array}$} & Amo \\
& & 31.83 & 2.286963 & 17.85650086 & 2.542 n.a. \\
$1 \mathrm{a}$ & & 37.61 & 0.172942 & 1.350326233 & 0.09947 n.a. \\
$2 \mathrm{~b}$ & & 72.94 & 10.06237 & 78.56651989 & 3.64837 n.a. \\
$3 \mathrm{c}$ & 94.55 & 0.285 & 2.226653014 & 0.132 n.a.
\end{tabular}




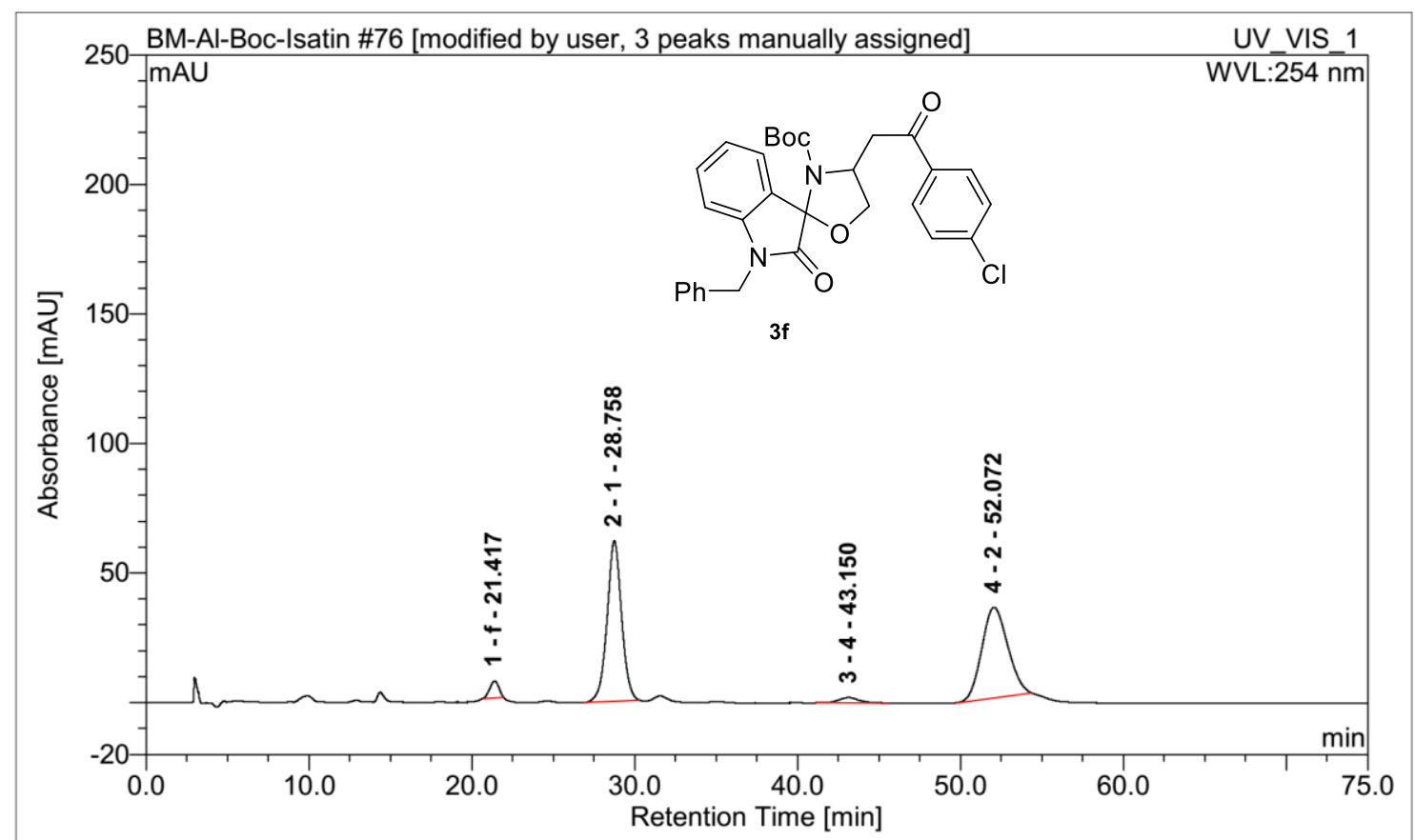

No.

$$
\begin{array}{ll}
\text { Peak Name } & \text { Ret. } \\
\text { min }
\end{array}
$$

Area

$\begin{array}{ll}1 & \mathrm{f} \\ 2 & 1 \\ 3 & 4 \\ 4 & 2\end{array}$
$m A U^{*} \min \%$

Rel.Area(ident.) Height

Amount $21.42 \quad 4.1003$ $28.76 \quad 64.08327$

$43.15 \quad 2.958201$
52.07

$3.022699227 \quad 6.43429$ n.a.

1

$52.07 \quad 64.509$

47.2415274361 .98517 n.a.

2.1807552112 .05932 n.a.

42 47.55501813



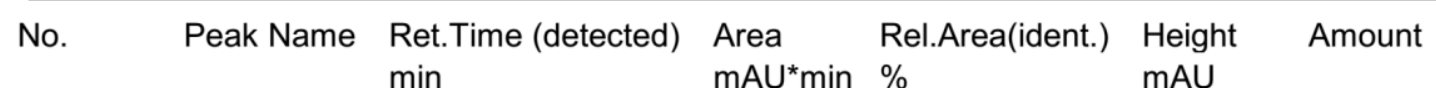

11

22

33

44
$21.12 \quad 210.7087$

28.679 .385412

$42.92 \quad 10.16221$

$51.04 \quad 450.869$
30.93537196242 .4915 n.a.

1.3779268659 .38932 n.a.

1.4919728327 .77108 n.a.

66.19472834213 .609 n.a. 




No. Peak Name Ret.Time (detected) Area Rel.Area(ident.) Height Amount $\min$ $\mathrm{mAU}{ }^{*} \min \%$
1 a
$2 \mathrm{~b}$
37.5233 .40626
36.0263436430 .18516 n.a.
3
$51.81 \quad 13.99952$
15.09751473
8.67935 n.a.
$4 \mathrm{~d}$
$97.28 \quad 13.2236$
14.26073882
3.9774 n.a.
$140.69 \quad 32.098$
34.61540282
7.779 n.a.

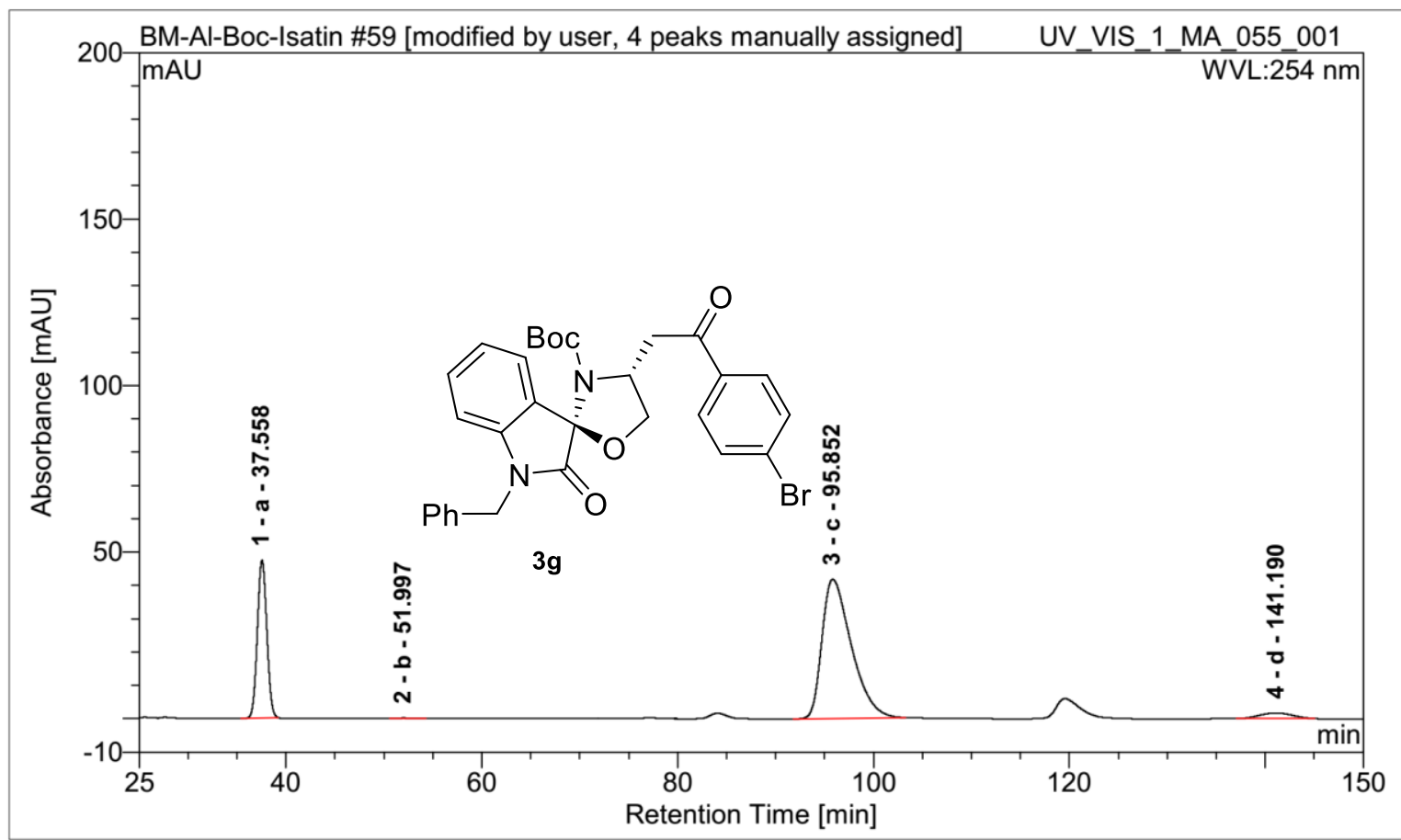

No.

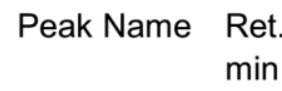

1 a

$2 \mathrm{~b}$

$3 \mathrm{c}$

$4 \mathrm{~d}$

$$
\min
$$

Rel.Area(ident.)

Area $\mathrm{mAU}{ }^{*} \min \%$
Amount

$\mathrm{mAU}$
$37.56 \quad 52.95415$

$52.00 \quad 0.218189$

$95.85 \quad 145.196$

141.19
6.513
25.84627914

0.1064955789

70.86840678

3.178818495
47.38682 n.a.

0.14375 n.a.

41.75586 n.a.

1.696 n.a. 


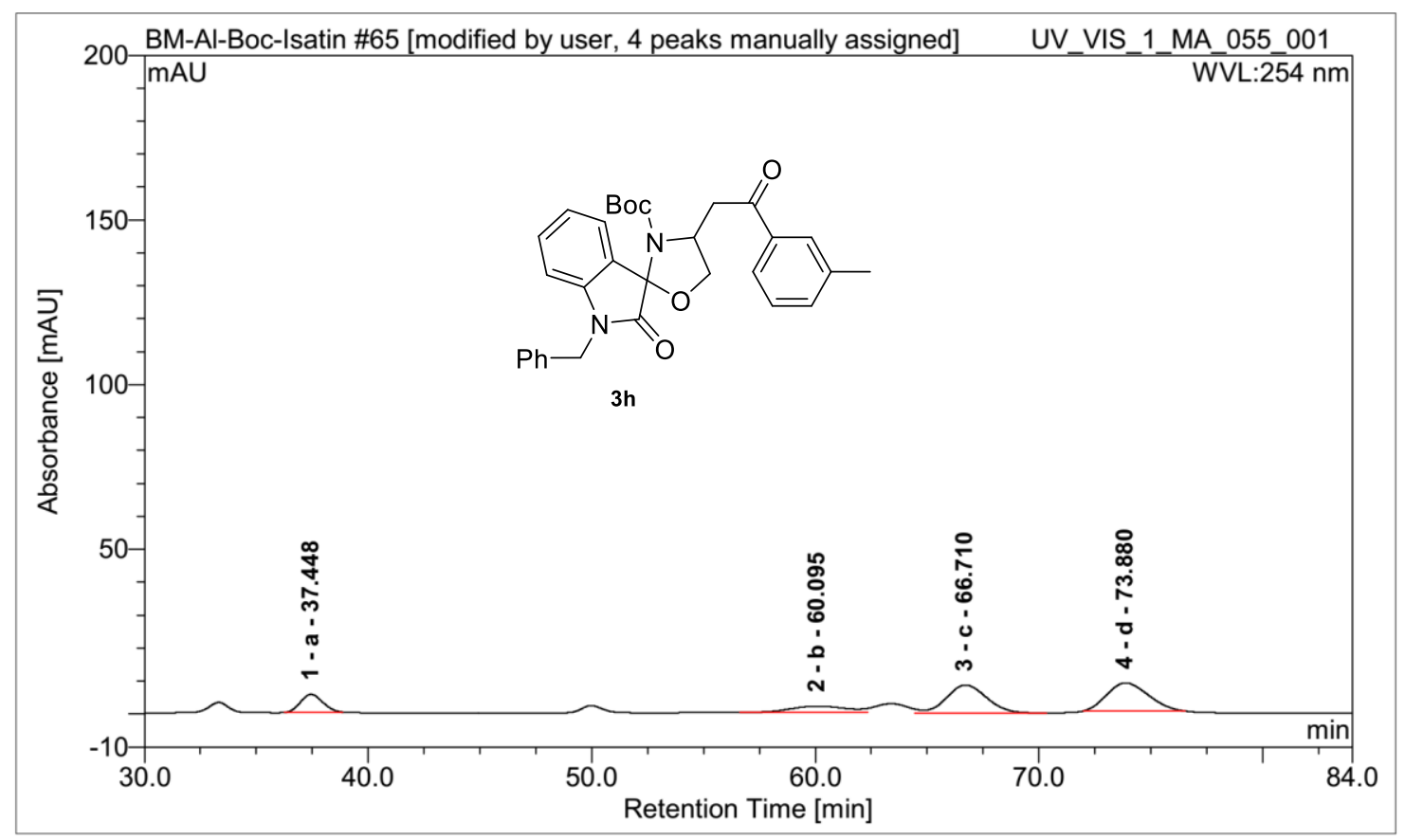

No. Peak Name Ret.Time (detected) Area Rel.Area(ident.) Height Amount $\min$ mAU*min \%

$\mathrm{mAU}$

$\begin{array}{lrrrr}1 \mathrm{a} & 37.45 & 6.237378 & 13.08106989 & 5.43451 \text { n.a. } \\ 2 \mathrm{~b} & 60.10 & 5.579069 & 11.7004599 & 1.86485 \text { n.a. } \\ 3 \mathrm{c} & 66.71 & 17.53206 & 36.76834843 & 8.41541 \text { n.a. } \\ 4 \mathrm{~d} & 73.88 & 18.334 & 38.45012177 & 8.552 \text { n.a. }\end{array}$






No. Peak Name Ret.Time (detected) Area Rel.Area(ident.) Height Amount min

$$
\mathrm{mAU}{ }^{*} \min \% \quad \mathrm{mAU}
$$

$\begin{array}{lrrrr}1 \mathrm{a} & 37.96 & 3.721845 & 5.795643684 & 3.22374 \text { n.a. } \\ 2 \mathrm{~b} & 57.18 & 19.33375 & 30.10644468 & 4.0755 \text { n.a. } \\ 3 \mathrm{c} & 66.35 & 40.47453 & 63.02678676 & 20.26321 \text { n.a. } \\ 4 \mathrm{~d} & 74.55 & 0.688 & 1.07112488 & 0.301 \text { n.a. }\end{array}$

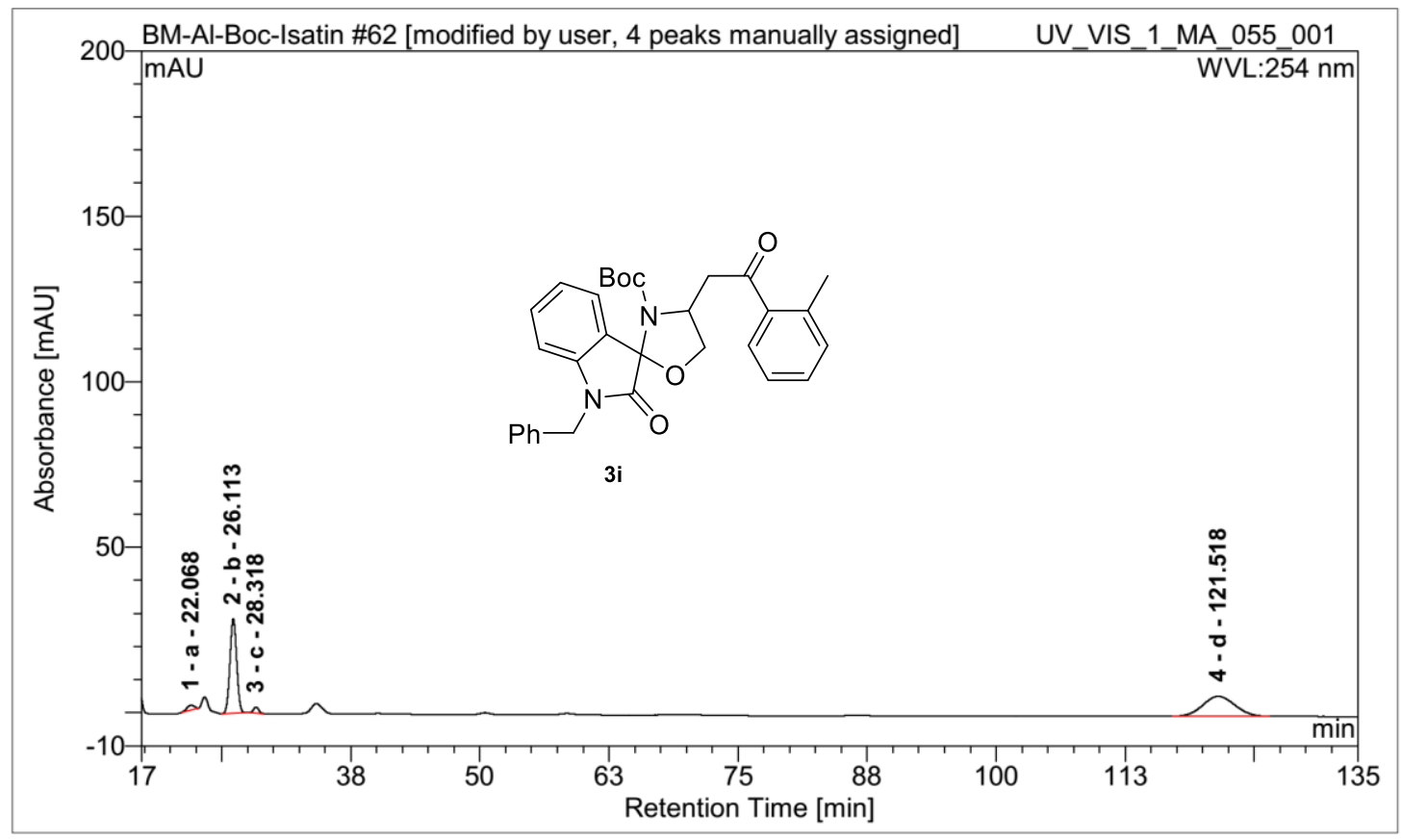

No. Peak Name Ret.Time (detected) Area Rel.Area(ident.) Height Amount $\min \quad \mathrm{mAU}^{*} \min \%$

$\begin{array}{rrrrr}1 \mathrm{a} & 22.07 & 1.269308 & 2.790723722 & 1.45595 \text { n.a. } \\ 2 \mathrm{~b} & 26.11 & 21.61254 & 47.51772434 & 28.54888 \text { n.a. } \\ 3 \mathrm{c} & 28.32 & 1.071658 & 2.356166179 & 1.84934 \text { n.a. } \\ 4 \mathrm{~d} & 121.52 & 21.530 & 47.33538576 & 6.021 \text { n.a. }\end{array}$




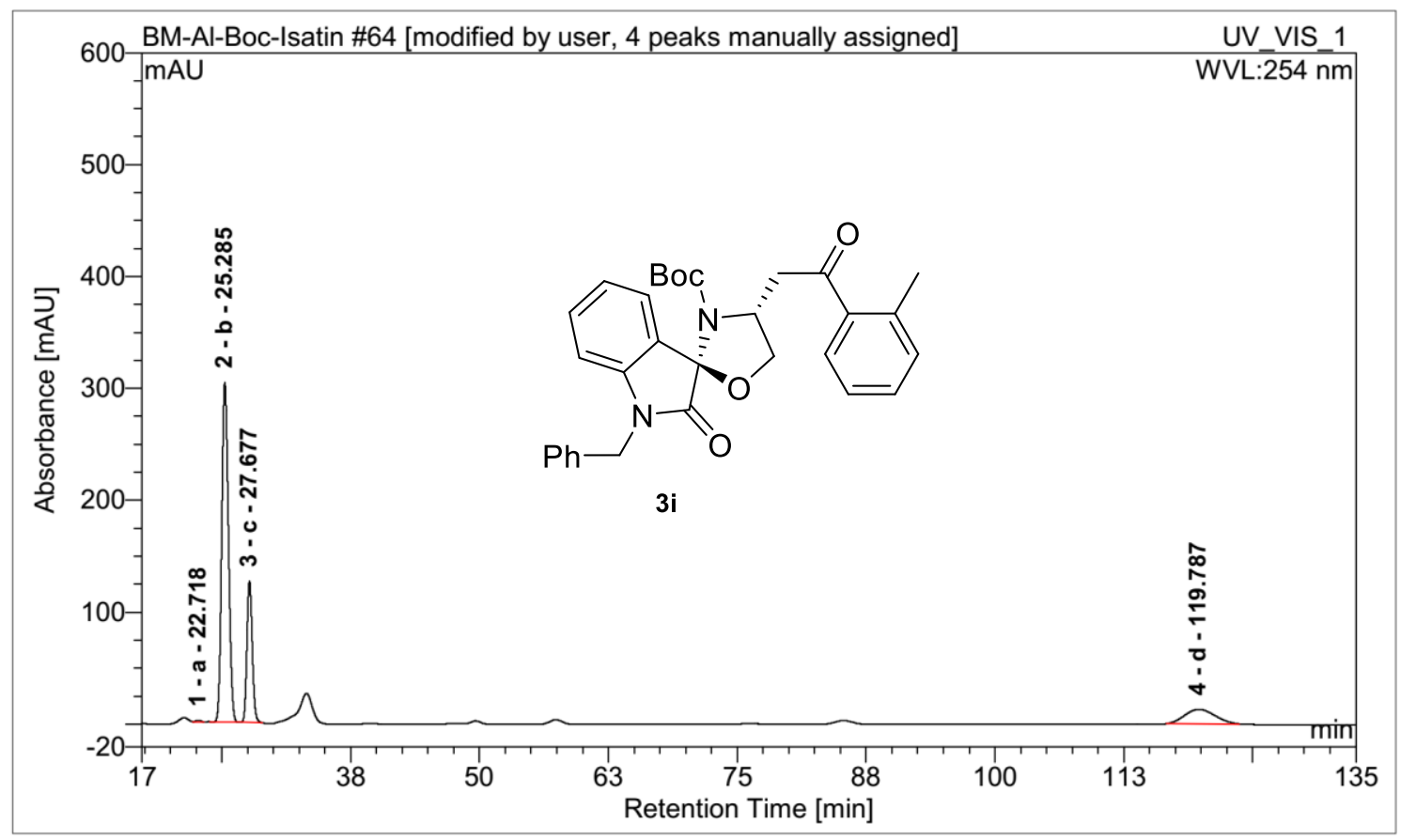

$\begin{array}{lllll}\text { No. Peak Name } & \text { Ret.Time (detected) } & \text { Area } & \text { Rel.Area(ident.) } & \text { Height } \\ & \text { min } & \text { mAU*min } \% & \text { mAU }\end{array}$ Amount

1 a

$2 \mathrm{~b}$

$3 \mathrm{c}$

$4 \mathrm{~d}$
22.720 .699007

$25.29 \quad 227.3966$

$27.68 \quad 75.5107$

$119.79 \quad 43.729$
0.20124852241 .38922 n.a.

65.46893873303 .2691 n.a.

21.74001381126 .3524 n.a.

$12.58979894 \quad 12.897$ n.a. 


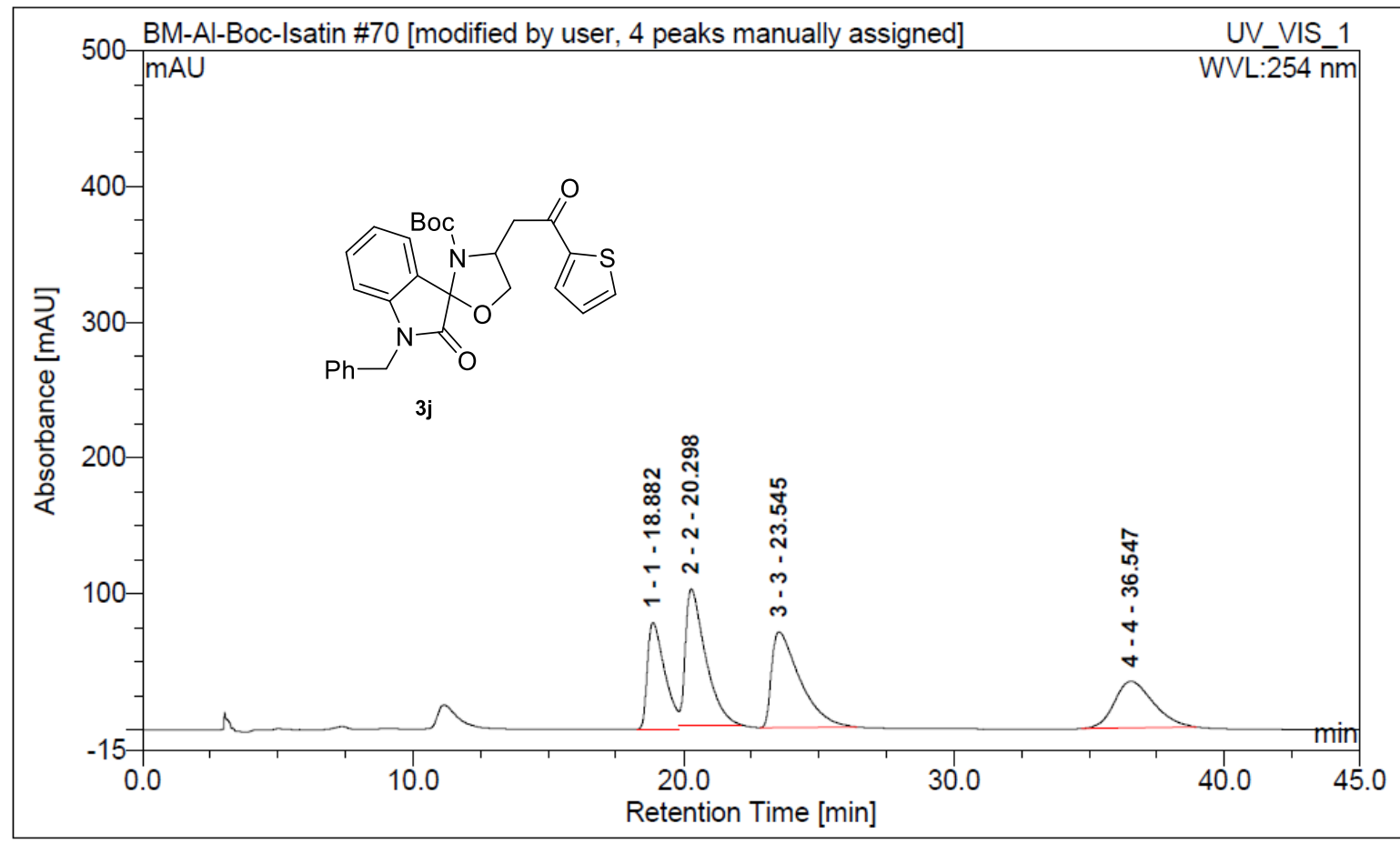

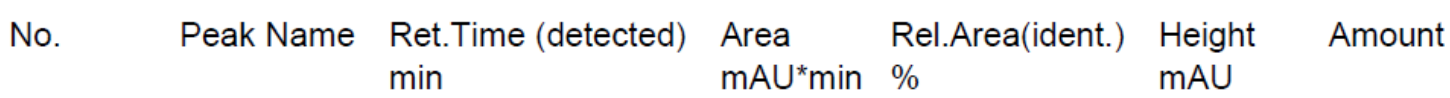
11
22
33
$18.88 \quad 58.23826$
20.3411768278 .40942 n.a.
$20.30 \quad 85.93774 \quad 30.01591595 \quad 99.90318$ n.a.
$\begin{array}{llll}23.55 & 85.20967 & 29.76161936 & 70.33026 \\ \text { n.a. }\end{array}$
44
$36.55 \quad 56.922$
$19.88128787 \quad 34.154$ n.a. 


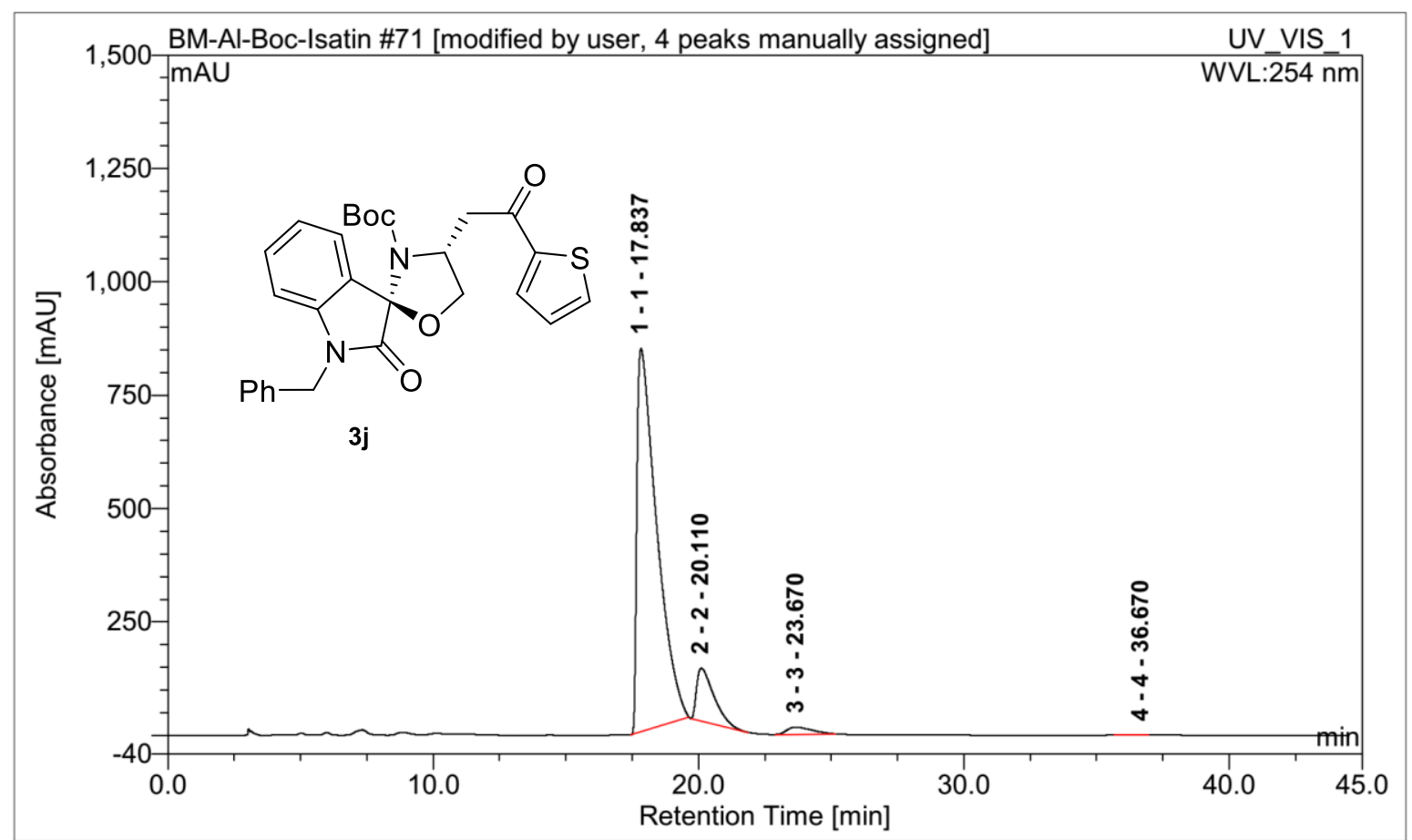

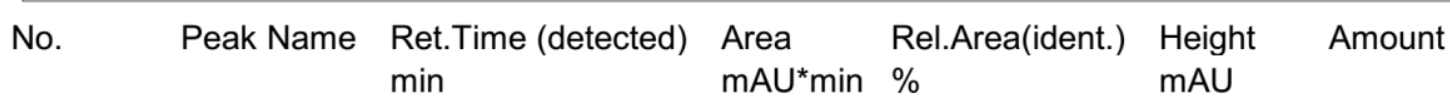
11
22
33
$17.84 \quad 730.402$
87.25731502844 .7597 n.a.
$\begin{array}{llll}20.11 & 89.30425 & 10.66871346 & 116.8453 \\ \text { n.a. }\end{array}$
44
$23.67 \quad 16.98927$
2.02961985915 .67393 n.a.
$\begin{array}{llll}36.67 & 0.371 & 0.04435165928 & 0.387\end{array}$ n.a.

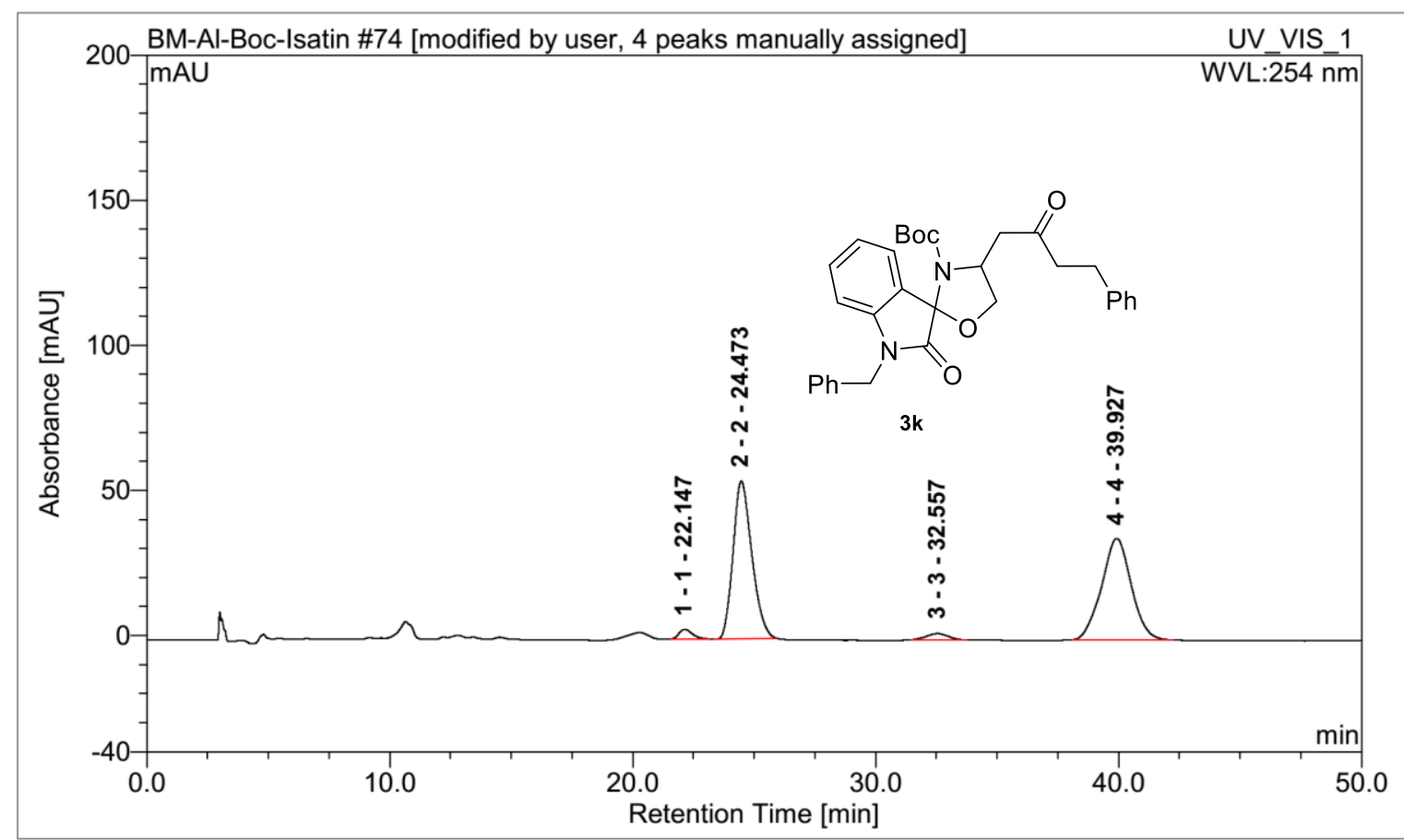

No. Peak Name Ret.Time (detected) Area Rel.Area(ident.) Height Amount $\min$ $\mathrm{mAU}^{*} \min \%$
11
22
33
22.152 .164678
$24.47 \quad 48.63266$
$32.56 \quad 2.307833$
44
$39.93 \quad 49.476$
$2.110201472 \quad 3.3474$ n.a.
47.4087565254 .31828 n.a.
$2.249753738 \quad 2.19686$ n.a.
$48.23128827 \quad 34.946$ n.a. 




No. Peak Name Ret.Time (detected) Area Rel.Area(ident.) Height Amount $\min \quad \mathrm{mAU}{ }^{*} \min \%$

31

42

53

64
$21.87 \quad 7.507996$

$24.10 \quad 62.02701$

31.8617 .84632

$39.55 \quad 4.737$
8.15038727611 .81455 n.a.

67.3341080870 .16789 n.a.

19.3732680217 .55022 n.a.

$5.142236625 \quad 3.733$ n.a. 


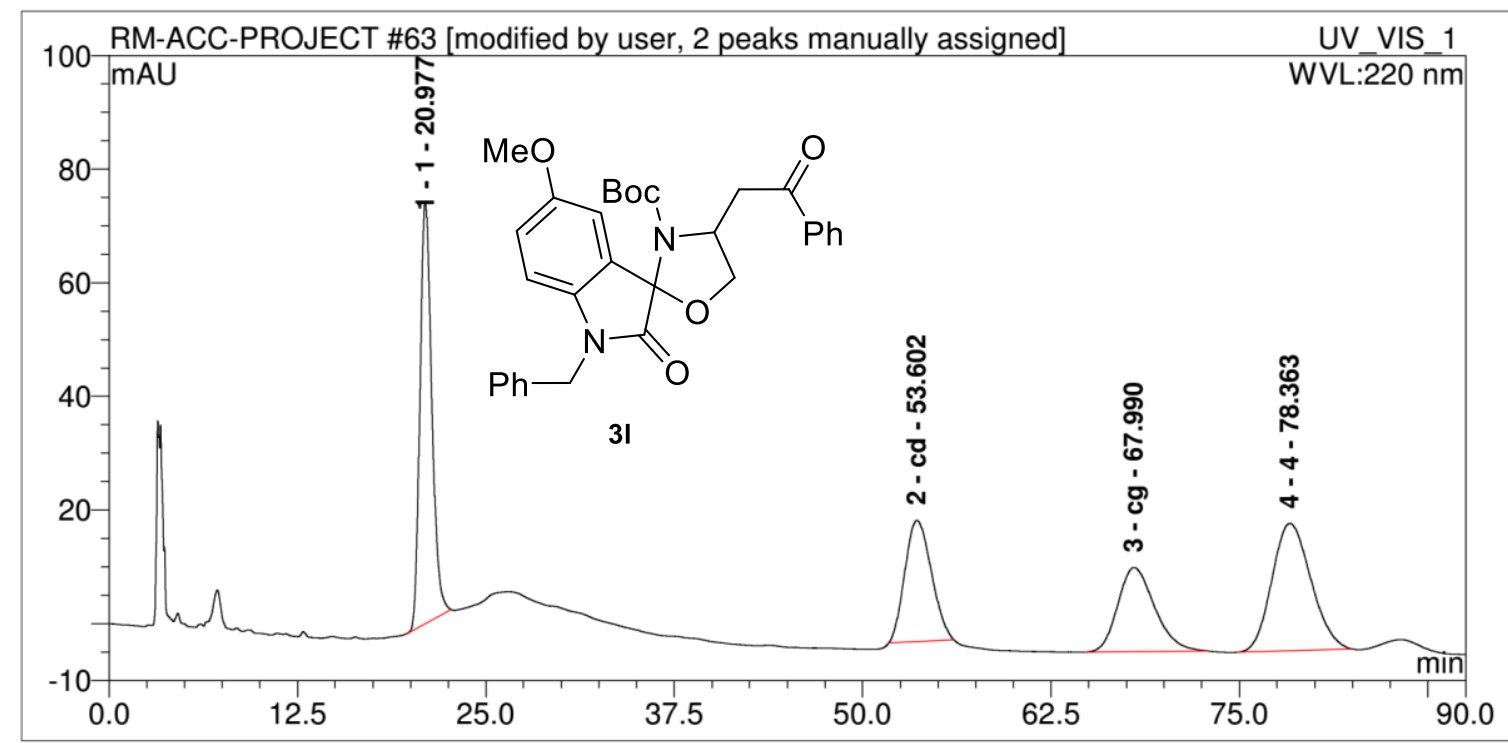

No.

\section{Peak Name}

11

$2 \mathrm{~cd}$

$3 \mathrm{cg}$

44
Ret

min

mA $\%$

20.9867 .26228

$53.60 \quad 43.58702$

67.9942 .04967

$78.36 \quad 67.314$
Rel.Area(ident.) Height Amount $\mathrm{mAU}$

30.5442206574 .22209 n.a.

19.7931365121 .37281 n.a.

19.0950146214 .82168 n.a.

$30.56762822 \quad 22.431$ n.a.



No.

Peak Name

$1 \mathrm{cc}$

22

33

44
Area $\mathrm{mAU}{ }^{*} \min \%$
Rel.Area(ident.) Height Amount $\mathrm{mAU}$
$20.96 \quad 140.799 \quad 98.80594148 \quad 153.5384$ n.a.

$\begin{array}{llll}50.05 & 1.308506 & 0.9182463127 & 1.33141 \\ \text { n.a. }\end{array}$

$\begin{array}{llll}68.47 & 0.052731 & 0.03700432396 & 0.06383 \\ \text { n.a. }\end{array}$

$\begin{array}{llll}78.90 & 0.340 & 0.2388078785 & 0.198\end{array}$ n.a. 


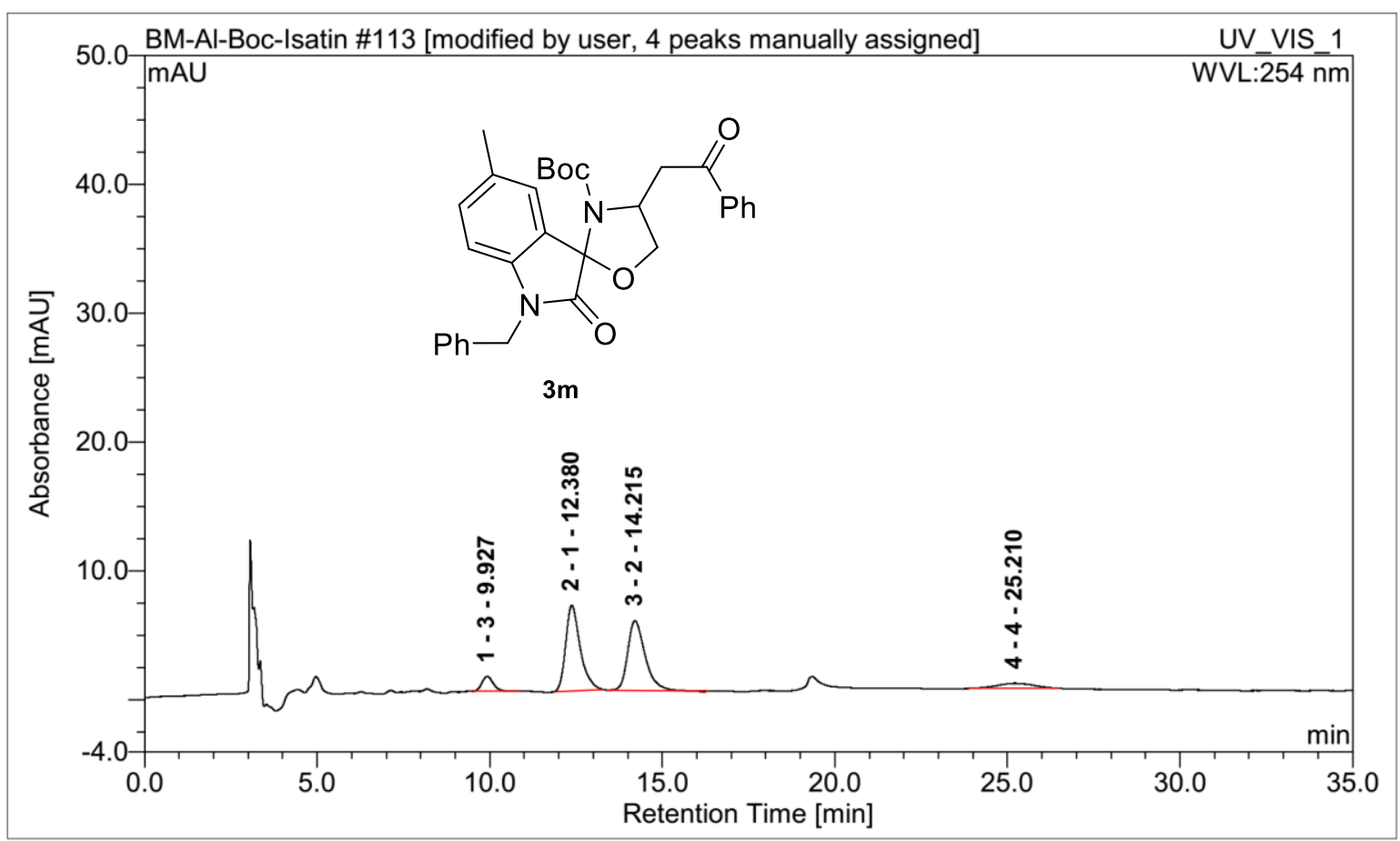

No. Peak Name Ret.Time (detected) Area Rel.Area(ident.) Height Amount $\min$ $\mathrm{mAU}^{*} \min \% \quad \mathrm{mAU}$
13
21
32
$9.93 \quad 0.443482$
6.0618940221 .15854 n.a.
44
$12.38 \quad 3.226056$
44.096486776 .62644 n.a.
$14.22 \quad 3.156641$
43.14766893
5.39718 n.a.
$25.21 \quad 0.490$
6.693950285
0.393 n.a.

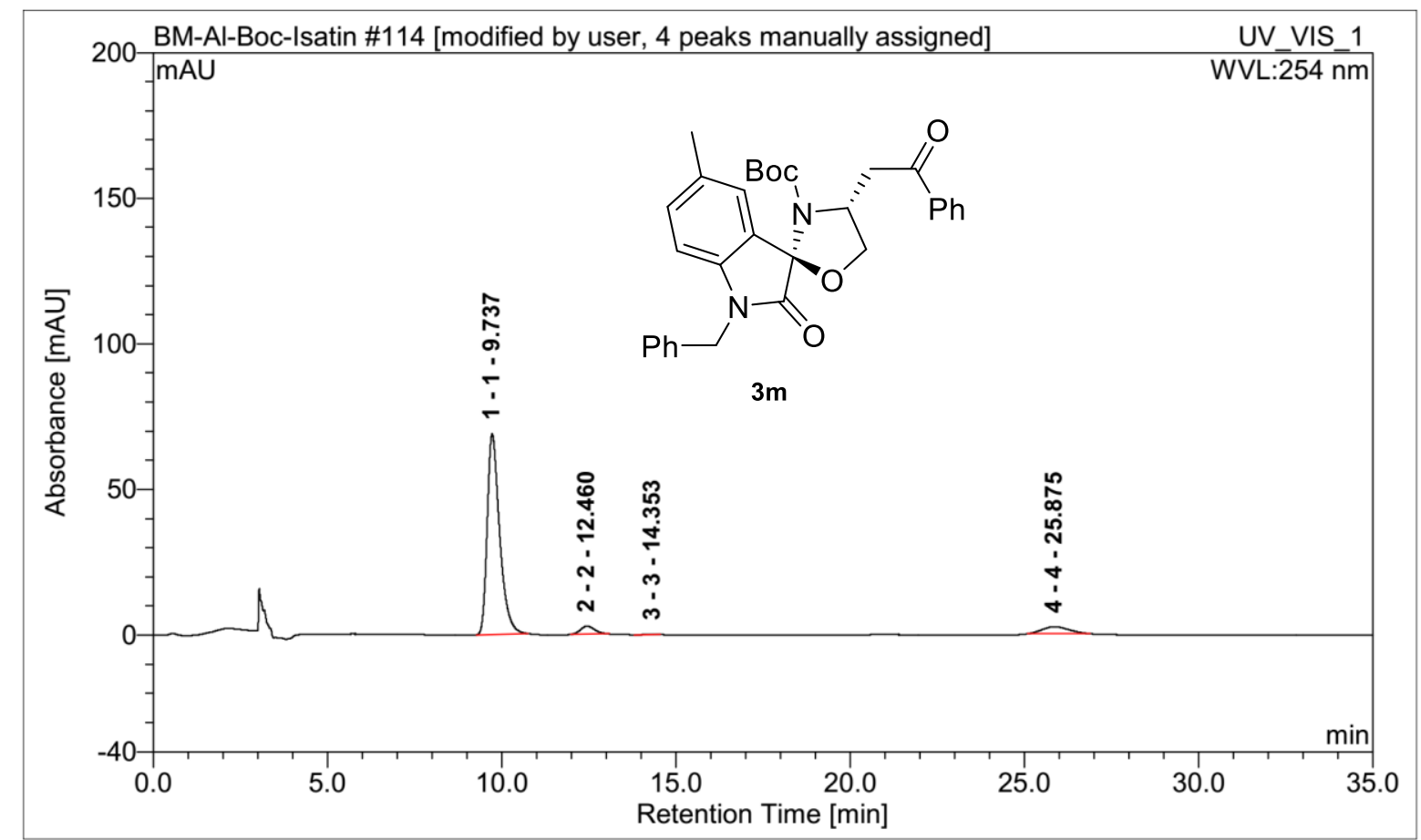

No. Peak Name Ret.Time (detected) Area Rel.Area(ident.) Height Amount $\min$ $\mathrm{mAU}^{*} \min \%$

$\mathrm{mAU}$
11
22
$9.74 \quad 28.41219$
$88.8331866 \quad 68.8777$ n.a.
33
$12.46 \quad 1.334684$
4.1730059572 .78904 n.a.
${ }^{4} 4$
$\begin{array}{ll}14.35 & 0.049405\end{array}$
0.1544701675
0.12536 n.a.
$25.88 \quad 2.187$
6.839337273
2.423 n.a. 




No.

Peak Name Ret.Time (detected) Area $\min$

$\begin{array}{ll}1 & 1 \\ 2 & 2 \\ 3 & 3 \\ 4 & 4\end{array}$ $\mathrm{mAU}{ }^{*} \min \%$

Rel.Area(ident.) Height Amount $\mathrm{mAU}$

$\begin{array}{rrrr}11.48 & 51.59398 & 12.75616988 & 114.7326 \text { n.a. } \\ 12.55 & 146.7492 & 36.28249176 & 289.3293 \text { n.a. } \\ 18.15 & 150.9611 & 37.32384773 & 169.4521 \text { n.a. } \\ 82.81 & 55.159 & 13.63749062 & 22.198 \text { n.a. }\end{array}$



No.

\section{Peak Name Ret.Time (detected)} $\min$

11

33

44

\section{Area} $\mathrm{mAU}^{*} \min \%$

$11.72 \quad 37.53368$

19.551 .261419

81.46
Rel.Area(ident.) Height

Amount $\mathrm{mAU}$

96.5346955478 .23084 n.a.

$3.244305637 \quad 1.39785$ n.a. $0.2209988204 \quad 0.079$ n.a. 


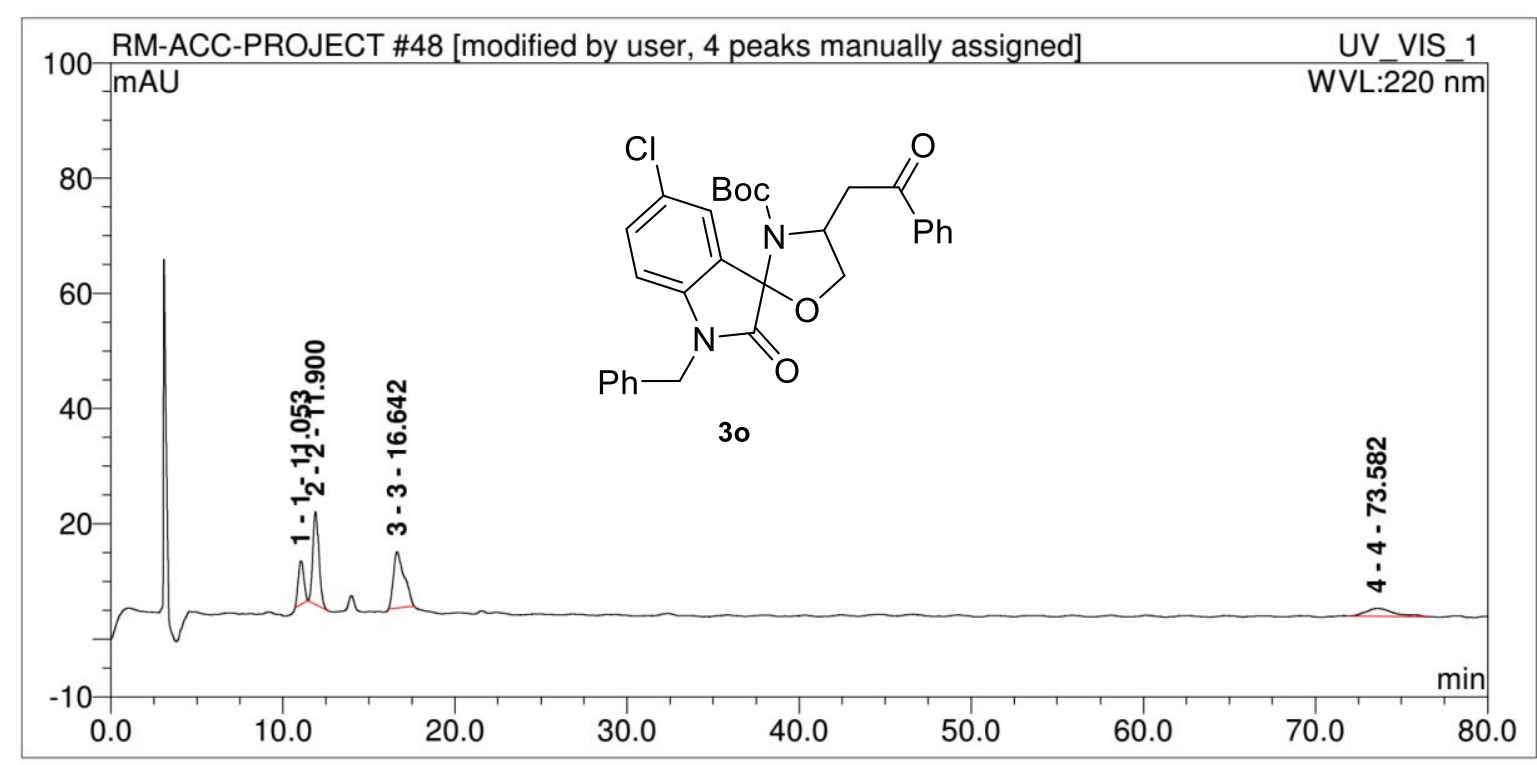

No.

Peak Name
11
22
33
44

Ret.
min me (det

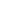

mAU*min \%

$11.05 \quad 2.83707$

$11.90 \quad 6.662333$

$\begin{array}{ll}16.64 & 7.083969\end{array}$

$\begin{array}{ll}73.58 & 2.574\end{array}$
Rel.Area(ident.) Height

$\mathrm{mAU}$
14.809175947 .56811 n.a.

34.7766083216 .09648 n.a.

36.977498019 .75366 n.a.

$13.43671774 \quad 1.368$ n.a.

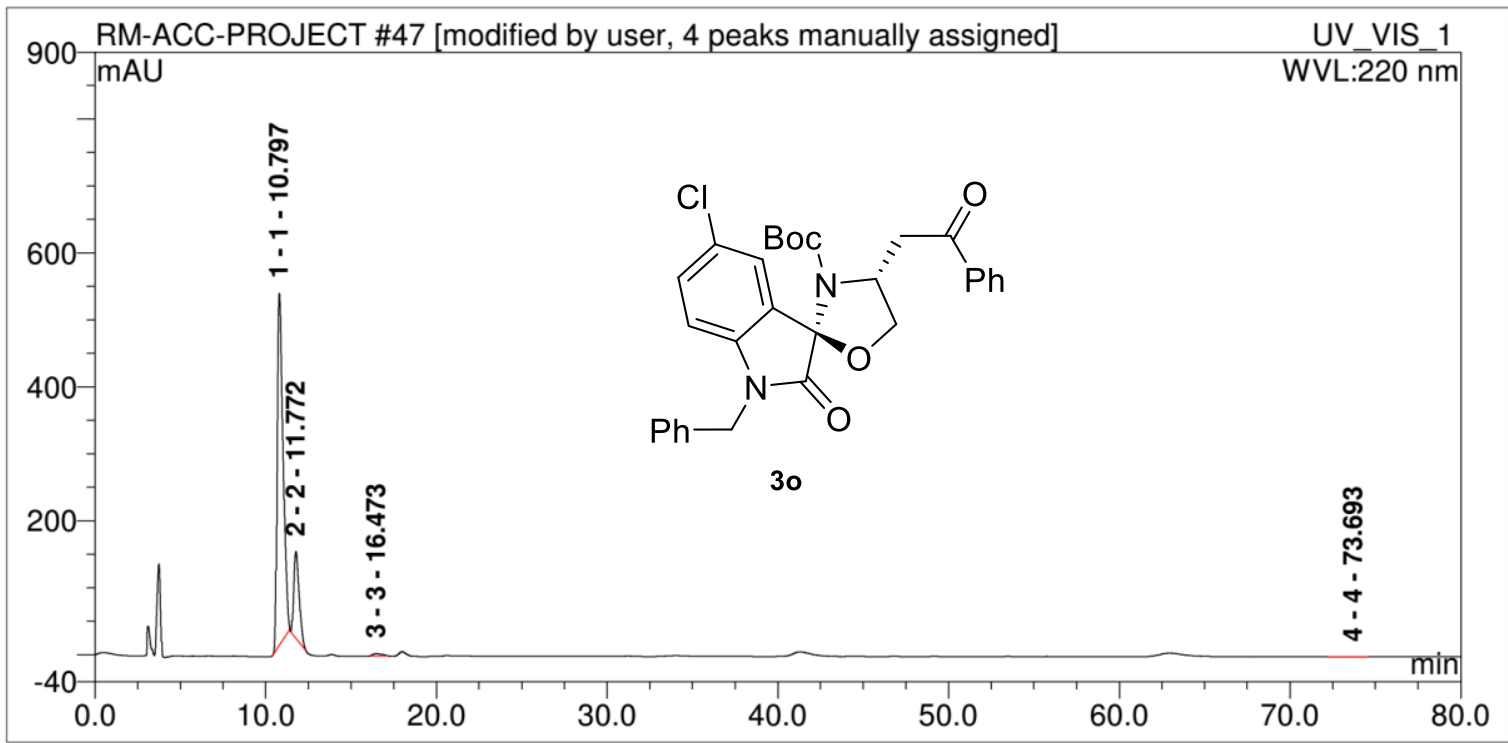

No.

\section{Peak Name}

11

22

33

44
Ret.
min
Area $\mathrm{mAU}^{*} \min \%$

$10.80 \quad 209.1626$

$11.77 \quad 47.83616$

$\begin{array}{ll}16.47 & 2.146972\end{array}$

73.69

0.129
Rel.Area(ident.) Height Amount $\mathrm{mAU}$
80.67234309525 .3035 n.a.

18.45002066129 .2573 n.a.

$0.8280699164 \quad 3.36084$ n.a.

0.195 n.a. 




No.

Peak Name

11

32

43

54
Rin

in

$250 \frac{\text { RM-ACC-PROJECT \#87 [modified by user, } 4 \text { peaks manually assigned] }}{-\mathrm{mAU}}$

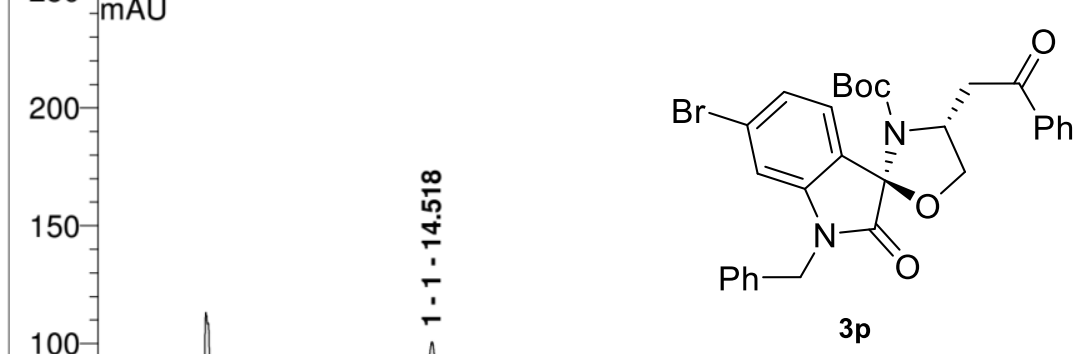

14.53111 .8582

Rel.Area(ident.)

22.29206 .9793

$28.96 \quad 96.02905$

$43.51 \quad 202.434$

Height

18.12053499129 .913 n.a.

33.52972618193 .1365 n.a.

15.5562793269 .54516 n.a.

Amount

$32.79345951 \quad 101.909$ n.a.

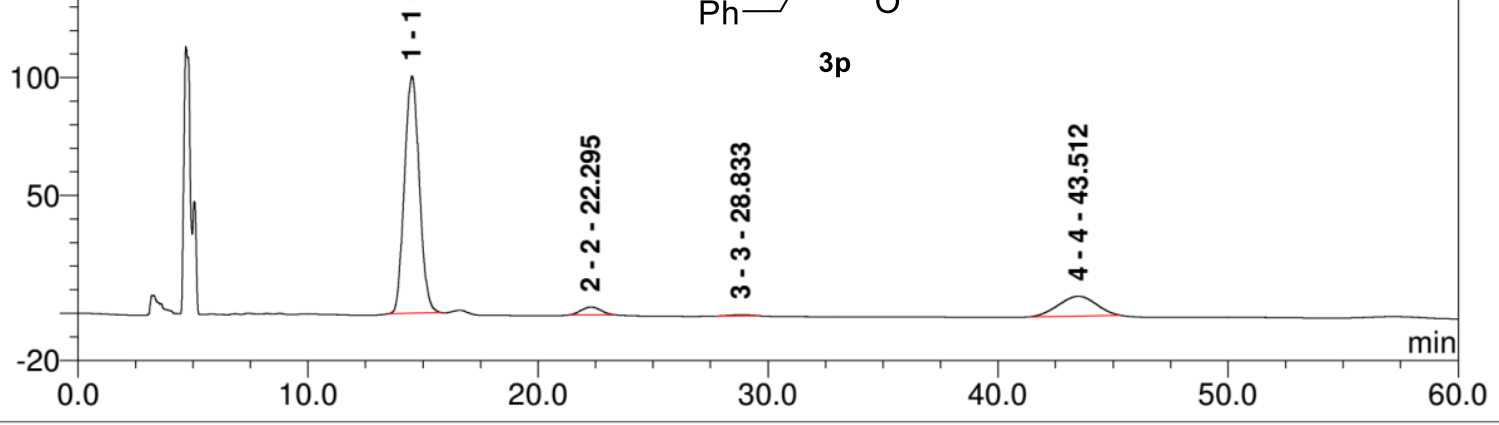

No.

Peak Name Ret.Time (detected) $\min$

11

22

33

44
Area $\mathrm{mAU}^{*} \min \%$ 14.5275 .76645 $22.30 \quad 3.275752$ $28.83 \quad 0.477623$ $43.51 \quad 15.792$
Rel.Area(ident.) Height Amount $\mathrm{mAU}$ 79.492893100 .7805 n.a. 3.4368638493 .31143 n.a. 0.50111455450 .45682 n.a.

The HPLC of the compound(3p) was taken and we observed only two peaks corresponding to the major diasteromer; there was no peak corresponding to the minor diasteromer. 




No.

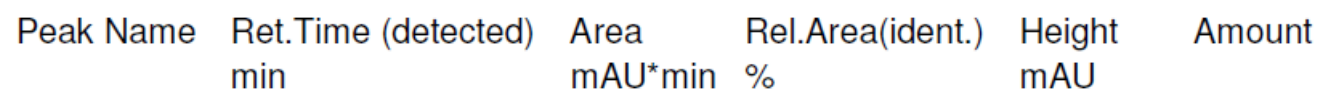
11
99.98647431132 .082 n.a.
22
29.28
$0.091 \quad 0.01352570398$
0.090 n.a. 


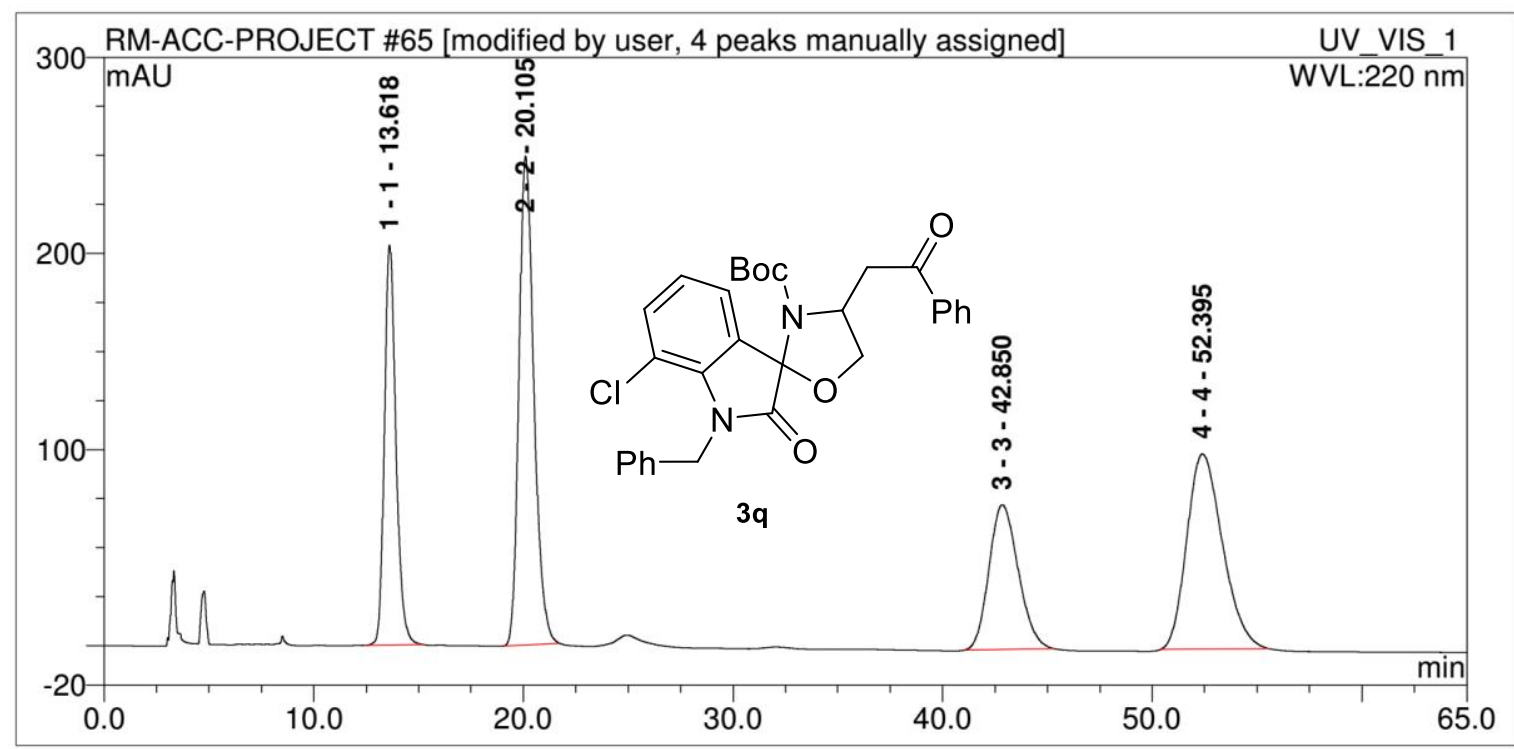

No.

Peak Name Ret.Time (detected) $\min$

11

22

33

44
Area $\mathrm{mAU}{ }^{*} \min \%$

13.62129 .9099

$20.11 \quad 205.806$

$42.85 \quad 119.3987$

$52.40 \quad 196.790$
Rel.Area(ident.) Height Amount $\mathrm{mAU}$

19.92776424203 .9377 n.a.

31.56997761249 .2066 n.a.

18.3153789673 .68561 n.a.

$30.18687919 \quad 99.579$ n.a.



No.

\section{$\begin{aligned} \text { Peak Name } & \text { Ret. } \\ & \text { min }\end{aligned}$}

11

22

33

44
Area

$\mathrm{mAU}{ }^{\star} \min \%$

13.63117 .2366

$20.23 \quad 2.921383$

$\begin{array}{ll}41.54 & 0.077719\end{array}$

52.67

14.672
Rel.Area(ident.) Height Amount $\mathrm{mAU}$

86.90134352190 .9312 n.a.

$2.165468611 \quad 3.6895$ n.a.

0.057608850840 .09617 n.a.

7.373 n.a. 


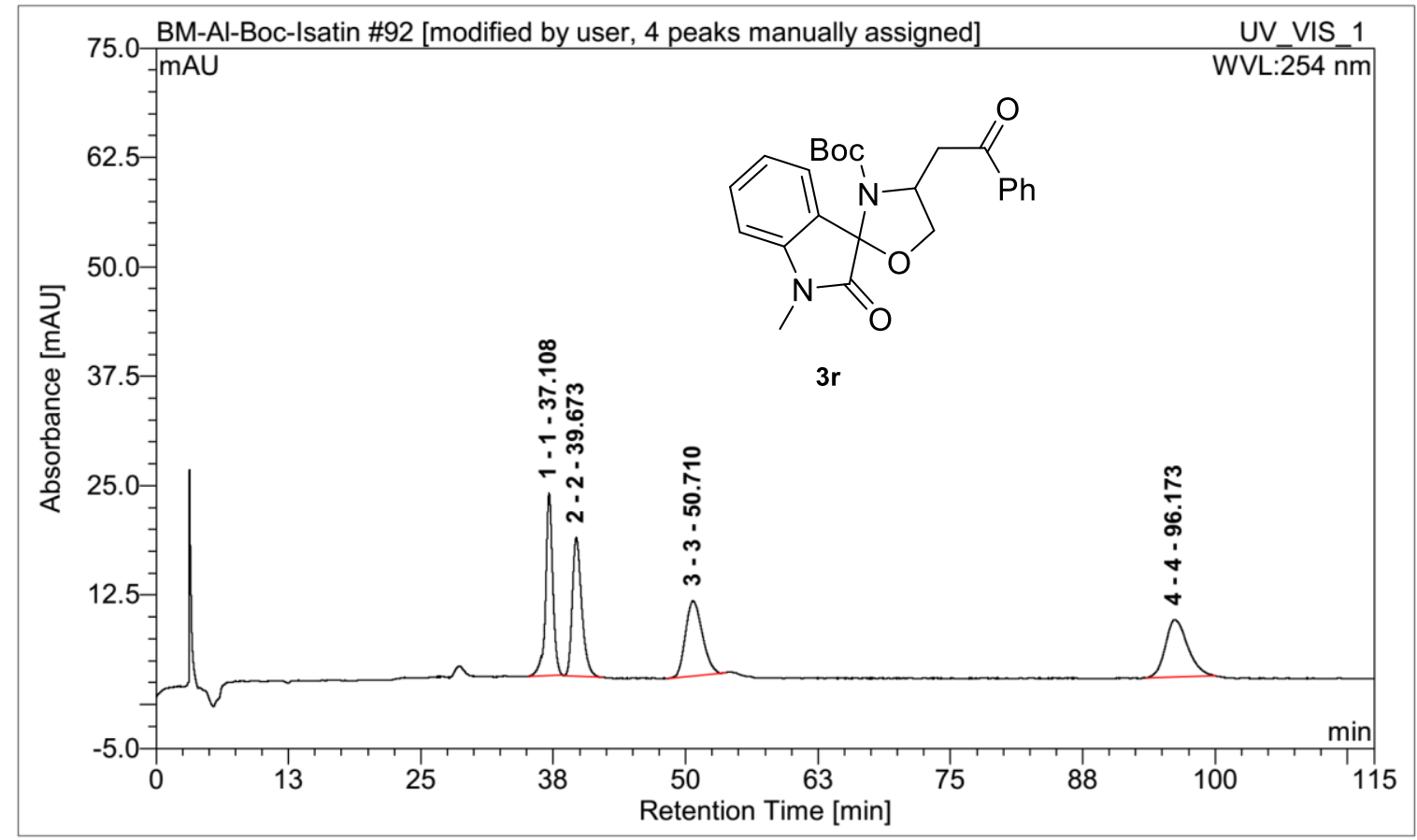

No. Peak Name Ret.Time (detected) Area Rel.Area(ident.) Height Amount
11
37.1116 .13264
25.0361187920 .79344 n.a.
22
39.6716 .38221
25.4234150715 .84059 n.a.
33
50.7115 .81898
24.54934844
8.57431 n.a.
44
96.17
16.104
24.99111769
6.542 n.a.



\begin{tabular}{|c|c|c|c|c|c|c|}
\hline No. & Peak Name & $\begin{array}{l}\text { Ret.Time (detected) } \\
\min \end{array}$ & $\begin{array}{l}\text { Area } \\
\mathrm{mAU}{ }^{\star} \min \end{array}$ & $\begin{array}{l}\text { Rel.Area(ident.) } \\
\%\end{array}$ & $\begin{array}{l}\text { Height } \\
\text { mAU }\end{array}$ & Amo \\
\hline & 11 & 37.02 & 34.73207 & 26.48075811 & 26.46386 & n.a. \\
\hline & 22 & 40.07 & 0.132823 & 0.1012685603 & 0.16609 & n.a. \\
\hline & 33 & 50.18 & 0.26604 & 0.2028368825 & 0.19549 & n.a \\
\hline & 44 & 93.06 & 96.029 & 73.21513645 & 33.570 & \\
\hline
\end{tabular}






\begin{tabular}{|c|c|c|c|c|c|c|}
\hline No. & Peak Name & $\begin{array}{l}\text { Ret.Time (detected) } \\
\text { min }\end{array}$ & $\begin{array}{l}\text { Area } \\
\mathrm{mA} U^{*} \min \end{array}$ & $\begin{array}{l}\text { Rel.Area(ident.) } \\
\%\end{array}$ & $\begin{array}{l}\text { Height } \\
\text { mAU }\end{array}$ & Amoun \\
\hline & 21 & 24.84 & 15.92797 & 17.76306863 & 23.04475 & n.a. \\
\hline & 32 & 33.41 & 15.04624 & 16.7797474 & 17.14352 & n.a. \\
\hline & 43 & 61.53 & 29.50862 & 32.90837235 & 17.29466 & n.a. \\
\hline & 54 & 68.26 & 29.186 & 32.54880535 & 14.949 & n.a. \\
\hline
\end{tabular}

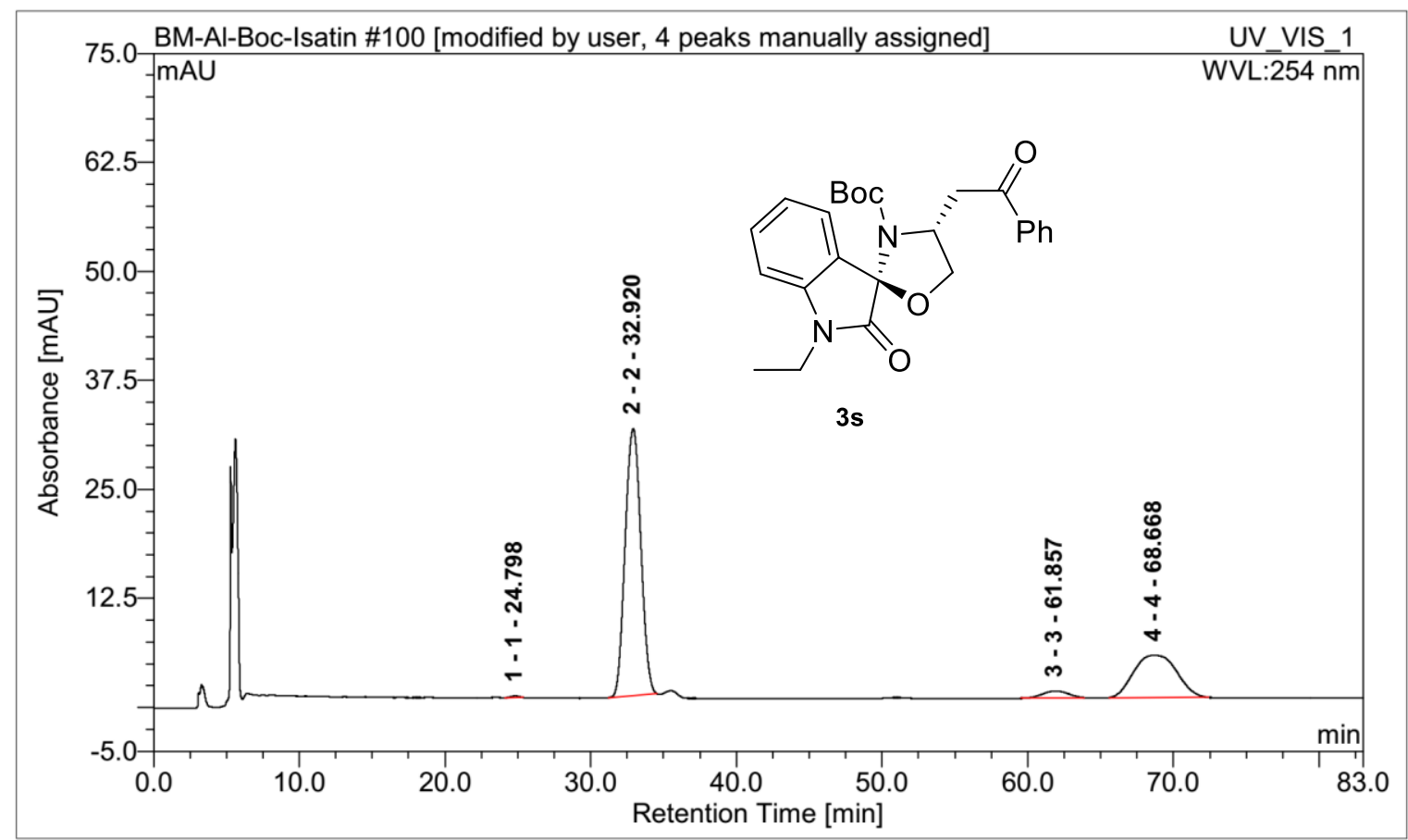

\begin{tabular}{|c|c|c|c|c|c|c|}
\hline No. & Peak Name & $\begin{array}{l}\text { Ret.Time (detected) } \\
\min \end{array}$ & $\begin{array}{l}\text { Area } \\
\mathrm{mAU}{ }^{*} \min \end{array}$ & $\begin{array}{l}\text { Rel.Area(ident.) } \\
\%\end{array}$ & $\begin{array}{l}\text { Height } \\
\text { mAU }\end{array}$ & Amount \\
\hline & 11 & 24.80 & 0.126315 & 0.2234122782 & 0.20919 & n.a. \\
\hline & 22 & 32.92 & 38.20469 & 67.57247629 & 30.6108 & n.a. \\
\hline & 33 & 61.86 & 1.608333 & 2.844652047 & 0.79978 & n.a. \\
\hline & 44 & 68.67 & 16.599 & 29.35945939 & 4.849 & n.a. \\
\hline
\end{tabular}






No.

\begin{tabular}{|c|c|c|c|c|c|}
\hline Peak Name & $\begin{array}{l}\text { Ret.Time (detected) } \\
\text { min }\end{array}$ & $\begin{array}{l}\text { Area } \\
\text { mAU*min }\end{array}$ & $\begin{array}{l}\text { Rel.Area(ident.) } \\
\%\end{array}$ & $\begin{array}{l}\text { Height } \\
\text { mAU }\end{array}$ & Amol \\
\hline 1 & 10.80 & 11.49911 & 9.424196593 & 22.58567 & n.a. \\
\hline 2 & 11.92 & 48.83371 & 40.02211683 & 86.80143 & n.a. \\
\hline 3 & 16.06 & 49.84397 & 40.85008318 & 101.4033 & n.a. \\
\hline 4 & 18.18 & 11.840 & 9.703603404 & 20.513 & n.a. \\
\hline
\end{tabular}



No.

Peak Name Ret.Time (detected) $\min$

11

44
Area

$\mathrm{mAU}{ }^{*} \min \%$
Rel.Area(ident.) Height Amount $\mathrm{mAU}$

$\begin{array}{llll}10.67 & 0.174367 & 0.1426063133 & 0.45748 \\ \text { n.a. }\end{array}$

$17.94 \quad 122.097 \quad 99.85739369205 .094$ n.a. 


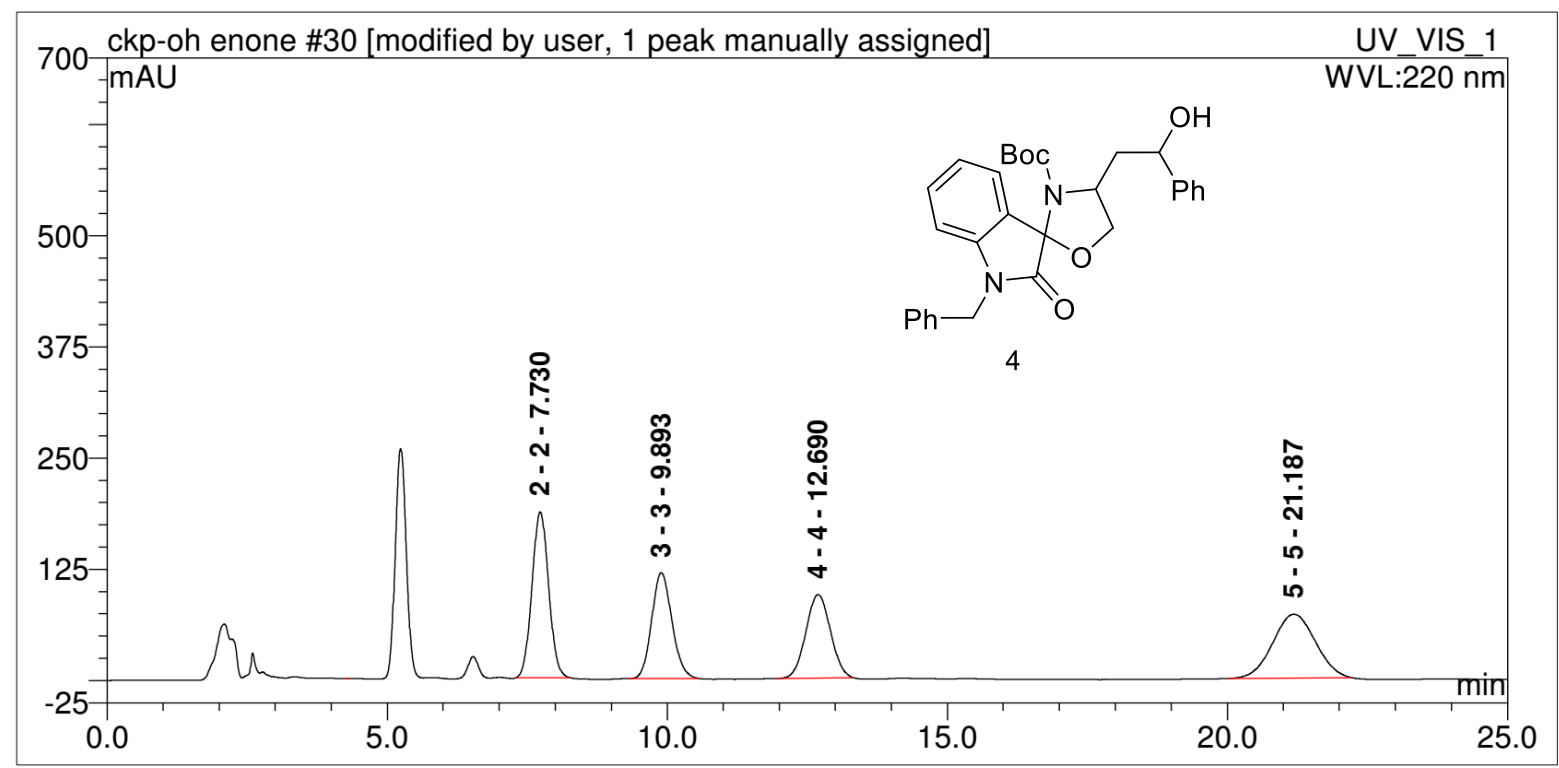

No.

Peak Name Ret.Time (detected) Area $\min$ $\mathrm{mAU}{ }^{*} \min \%$

Rel.Area(ident.) Height Amount $7.73 \quad 64.40954$ 9.8950 .01692 12.6947 .67819 $21.19 \quad 63.148$ 28.59432292186 .3859 n.a. 22.20478455118 .8388 n.a. 21.1665170693 .85453 n.a. $28.03437547 \quad 71.689$ n.a.

22

33

44

55

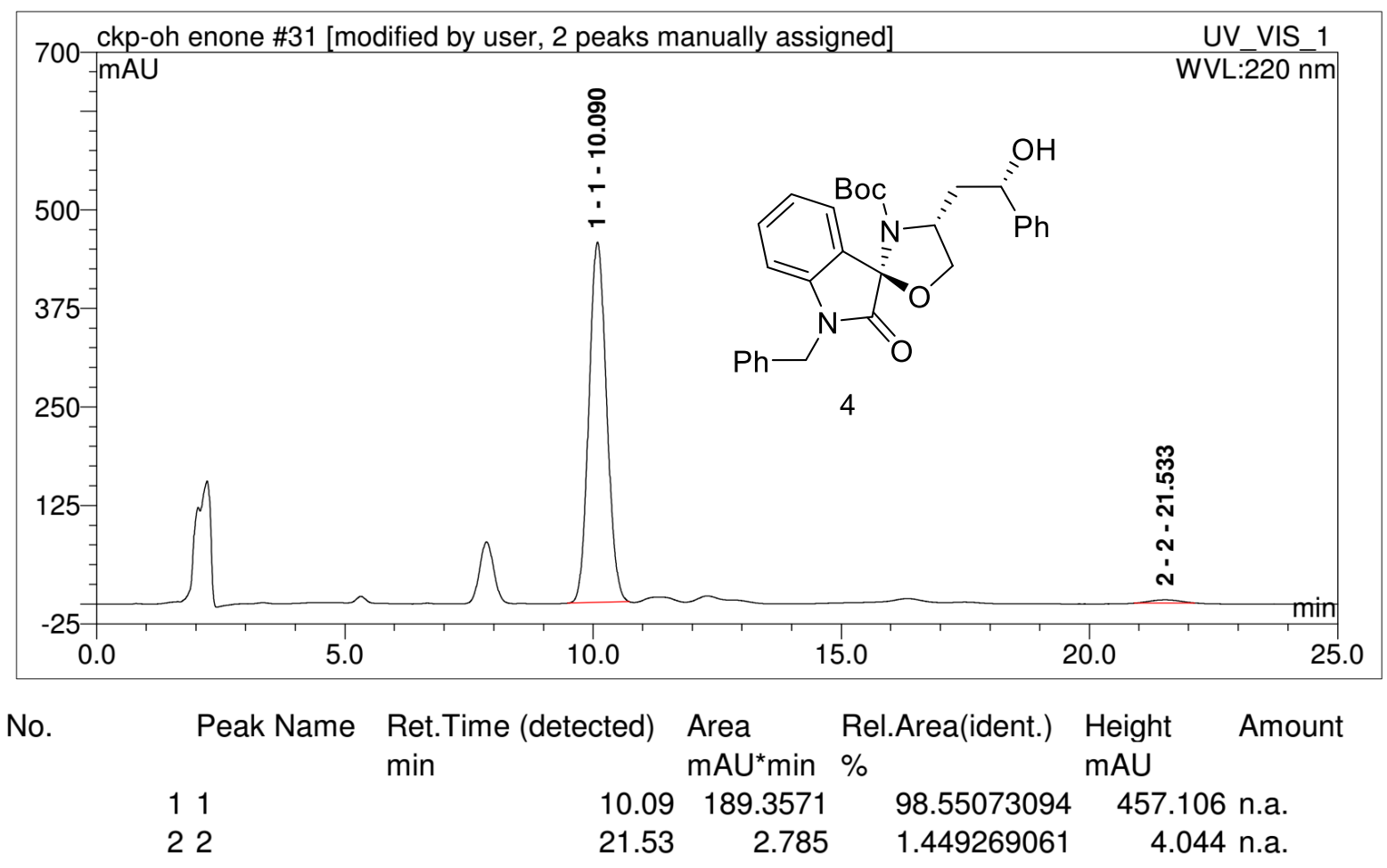






No.

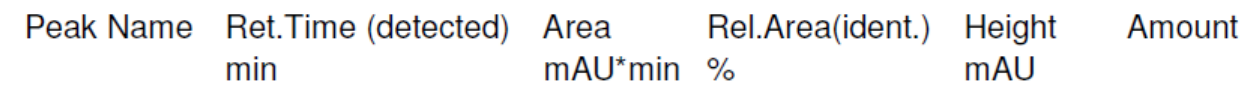
11
22
$4.96 \quad 110.7246$
25.05784432363 .3501 n.a
33
$\begin{array}{ll}6.58 & 110.6067\end{array}$
25.03115618415 .5862 n.a.
44
$8.66 \quad 109.009$
24.66957083298 .147 n.a.
$10.35 \quad 111.536 \quad 25.24142867 \quad 250.526$ n.a.

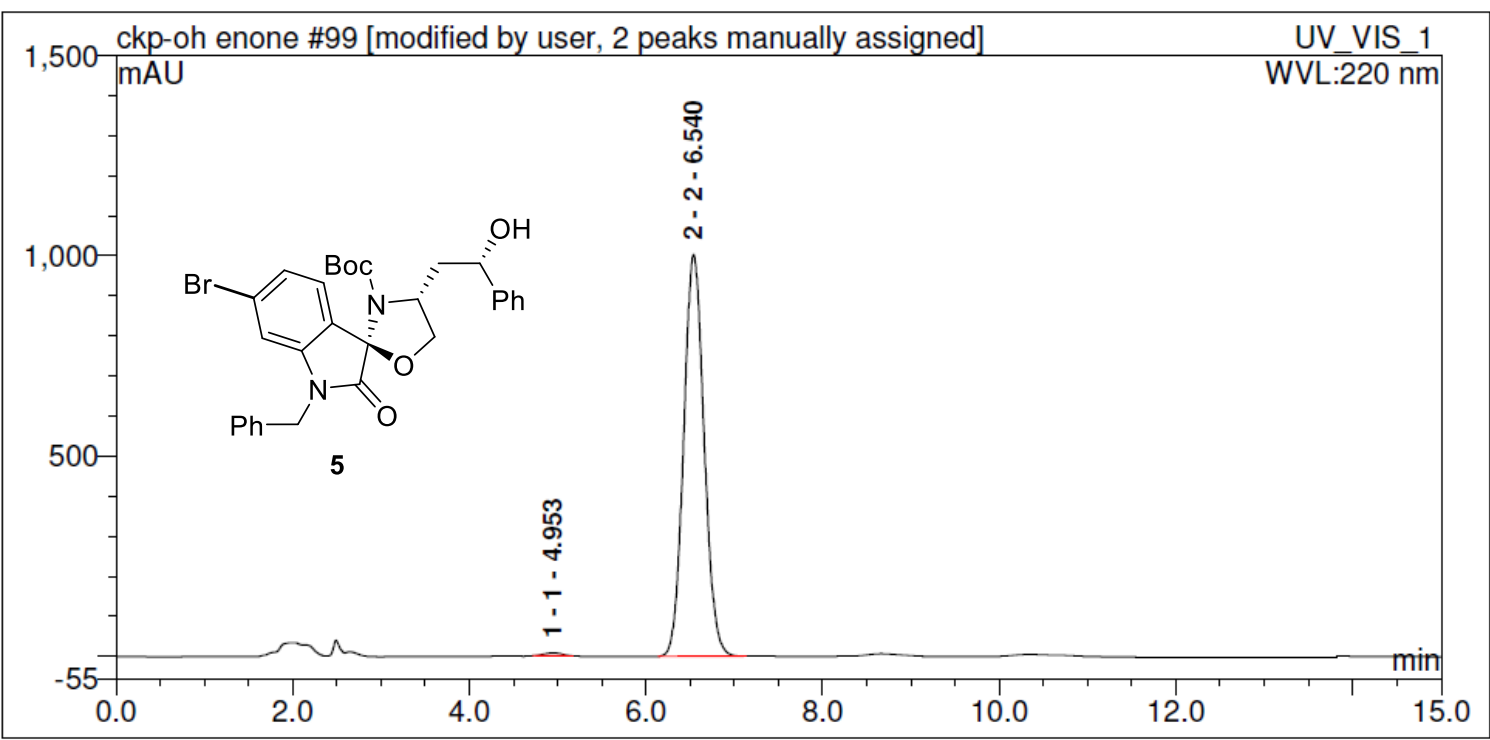

No.

Peak Name Ret.Time (detected)

Area $\mathrm{mAU} U^{*} \min \%$

$4.95 \quad 1.663555$

$6.54 \quad 268.555$
Rel.Area(ident.) Height Amount $\mathrm{mAU}$
11

22
$0.6156326299 \quad 6.782$

99.384367371002 .824 n.a. 




No.

Peak Name Ret.Time (detected) $\min$

Rel.Area(ident.) Height Amount mAU*min \%

$\mathrm{mAU}$

11

16.642 .052814

$6.628812307 \quad 2.94071$ n.a.

22

$20.67 \quad 13.34265$

43.08518929

16.76154 n.a.

33

25.492 .190205

44

$39.08 \quad 13.382$

7.072464162

2.61585 n.a.

43.21353424

9.248 n.a.



No.

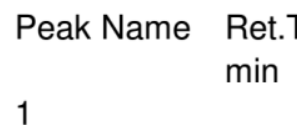

11

22

$\min$
Area $\mathrm{mAU}{ }^{*} \min \%$
Rel.Area(ident.) Height $\mathrm{mAU}$ $\begin{array}{llll}16.45 & 217.5679 & 99.96782372 & 297.6209 \\ \text { n.a. }\end{array}$

$25.00 \quad 0.070 \quad 0.03217627585 \quad 0.017$ n.a. 


\section{HRMS spectra of the products:}

HRMS (ESI) m/z: [M + Na] ${ }^{+}$calcd. for 3a $\mathrm{C}_{30} \mathrm{H}_{30} \mathrm{~N}_{2} \mathrm{O}_{5} \mathrm{Na}$ 521.2047, found 521.2048.



HRMS (ESI) m/z: $[\mathrm{M}+\mathrm{Na}]^{+}$calcd. for $3 \mathbf{b} \mathrm{C}_{31} \mathrm{H}_{32} \mathrm{~N}_{2} \mathrm{O}_{5} \mathrm{Na} 535.2203$, found 535.2203.

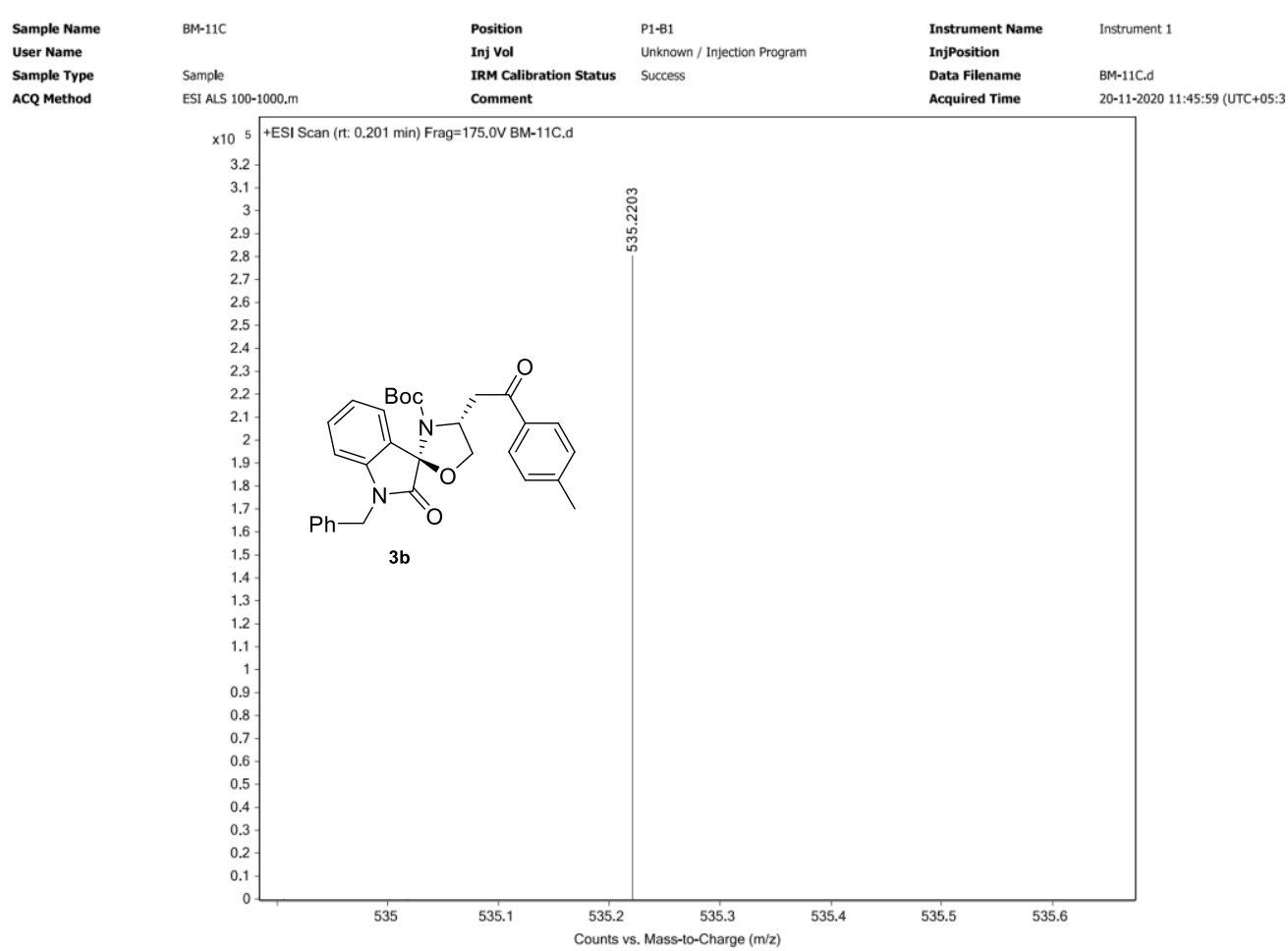


HRMS (ESI) m/z: $[\mathrm{M}+\mathrm{Na}]^{+}$calcd. for $3 \mathbf{c} \mathrm{C}_{33} \mathrm{H}_{36} \mathrm{~N}_{2} \mathrm{O}_{5} \mathrm{Na}$ 563.2516, found 563.2515.



HRMS (ESI) m/z: $[\mathrm{M}+\mathrm{Na}]^{+}$calcd. for $3 d \mathrm{C}_{34} \mathrm{H}_{38} \mathrm{~N}_{2} \mathrm{O}_{5} \mathrm{Na}$ 577.2673, found 577.2674.

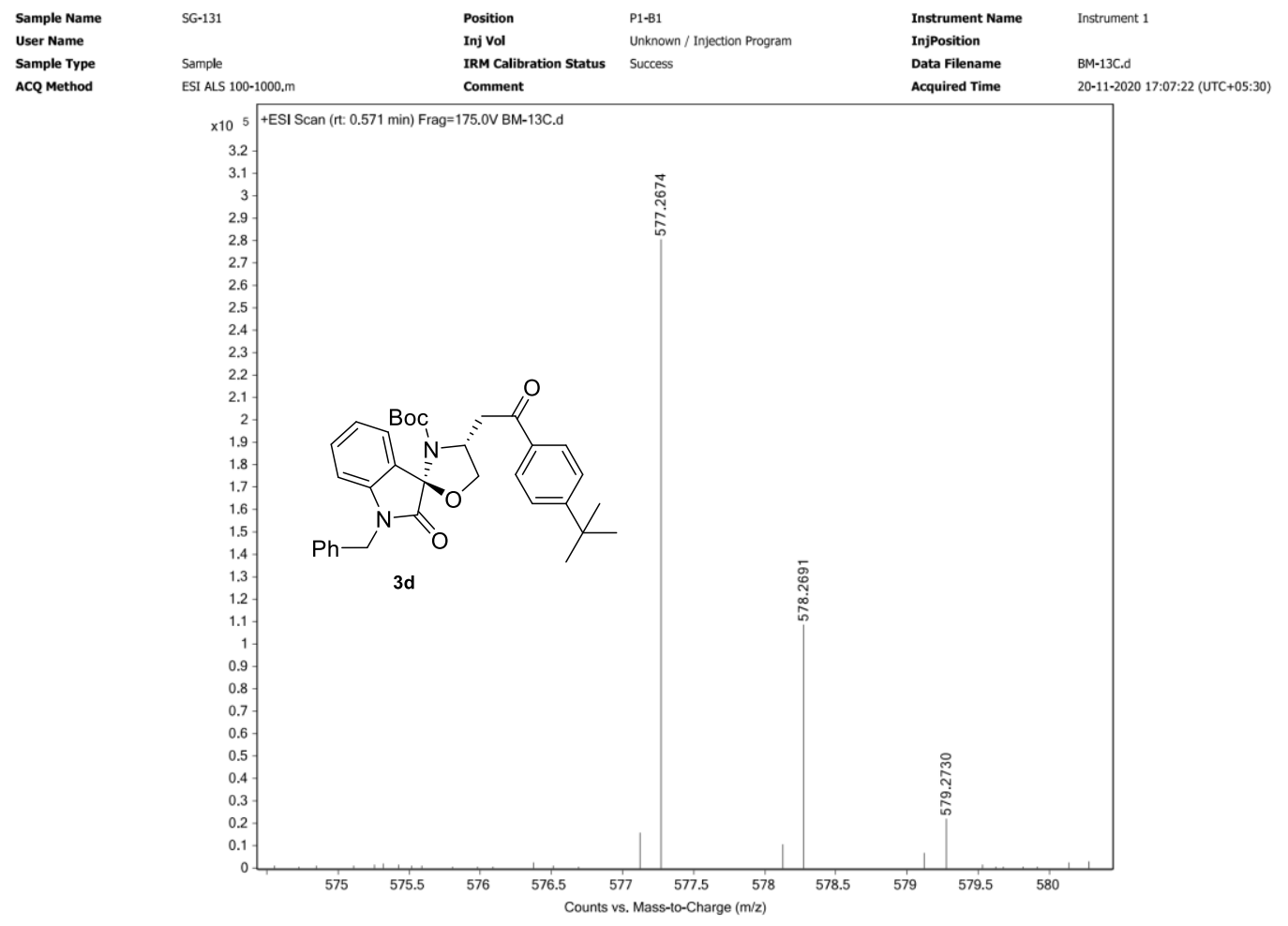


HRMS (ESI) m/z: [M + Na $]^{+}$calcd. for $3 \mathrm{e}_{30} \mathrm{H}_{29} \mathrm{FN}_{2} \mathrm{O}_{5} \mathrm{Na}$ 539.1953, found 539.1957.

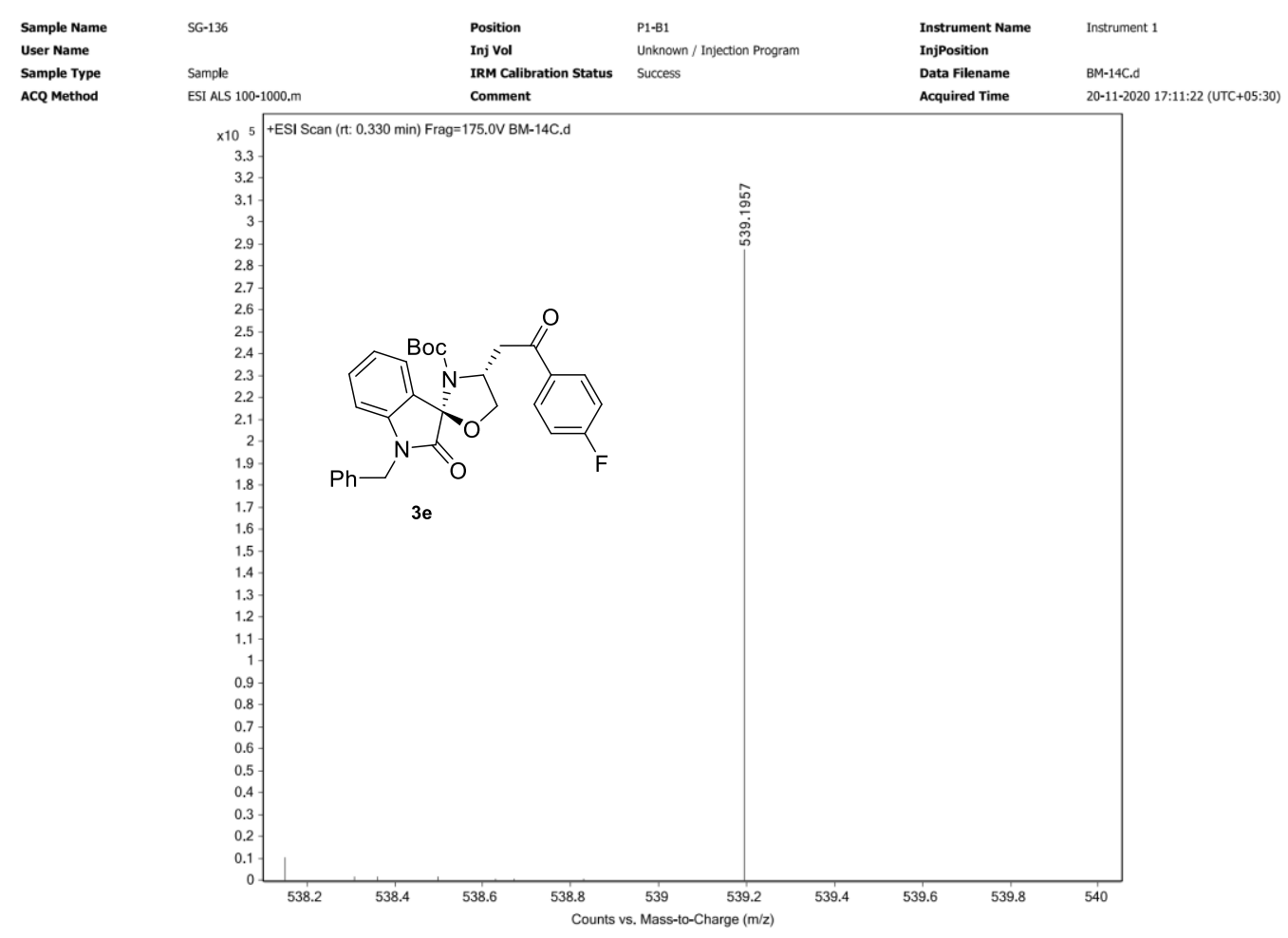

HRMS (ESI) m/z: [M + Na $]^{+}$calcd. for $3 f \mathrm{C}_{30} \mathrm{H}_{29} \mathrm{ClN}_{2} \mathrm{O}_{5} \mathrm{Na} 555.1657$, found 555.1658.




HRMS (ESI) m/z: [M + Na] ${ }^{+}$calcd. for 3g C $30 \mathrm{H}_{29} \mathrm{BrN}_{2} \mathrm{O}_{5} \mathrm{Na} 599.1152$, found 599.1171.

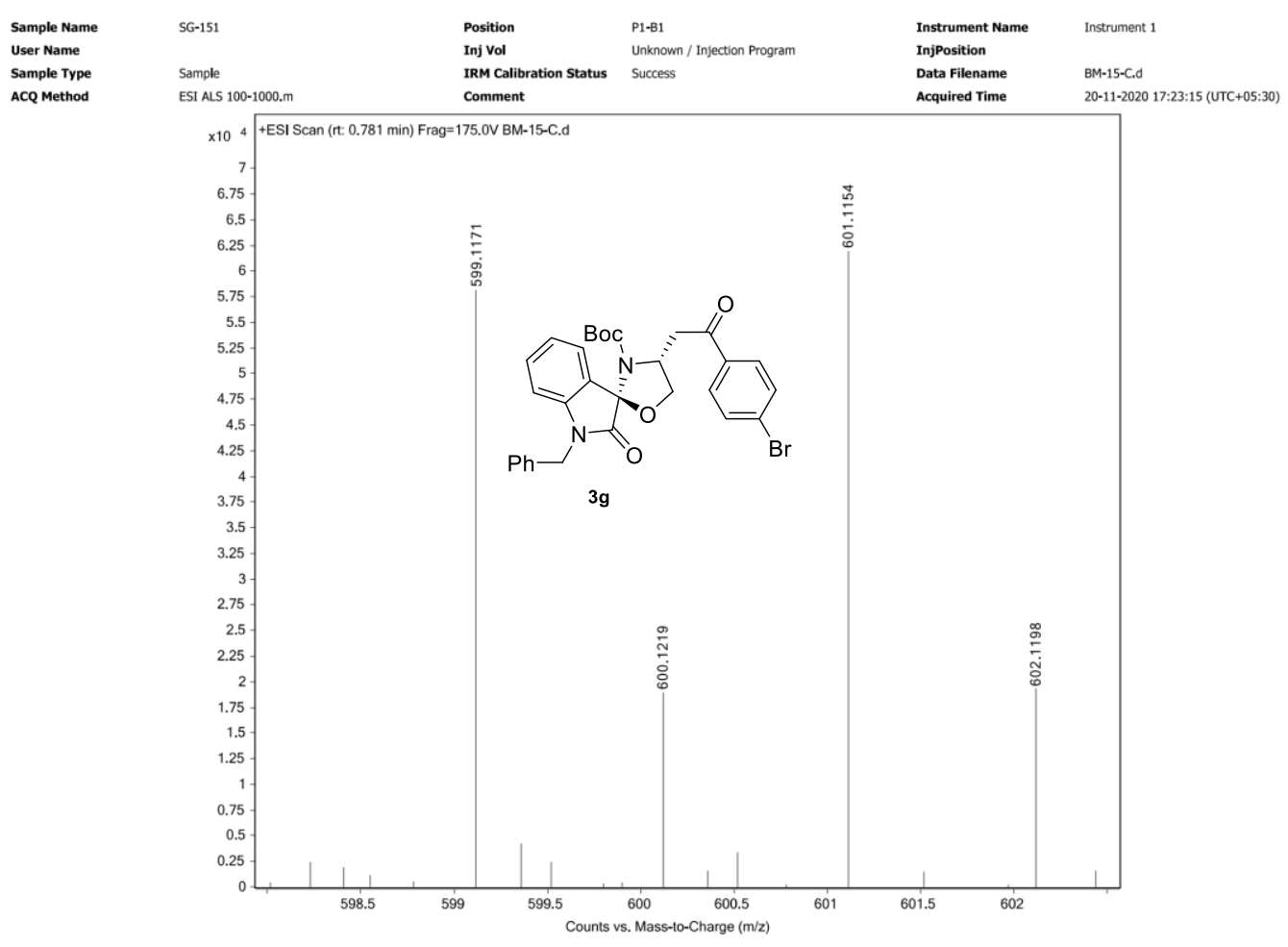

HRMS (ESI) m/z: [M + Na $]^{+}$for $\mathbf{3 h ~} \mathrm{C}_{31} \mathrm{H}_{32} \mathrm{~N}_{2} \mathrm{O}_{5} \mathrm{Na}$ 535.2203, found 535.2214.

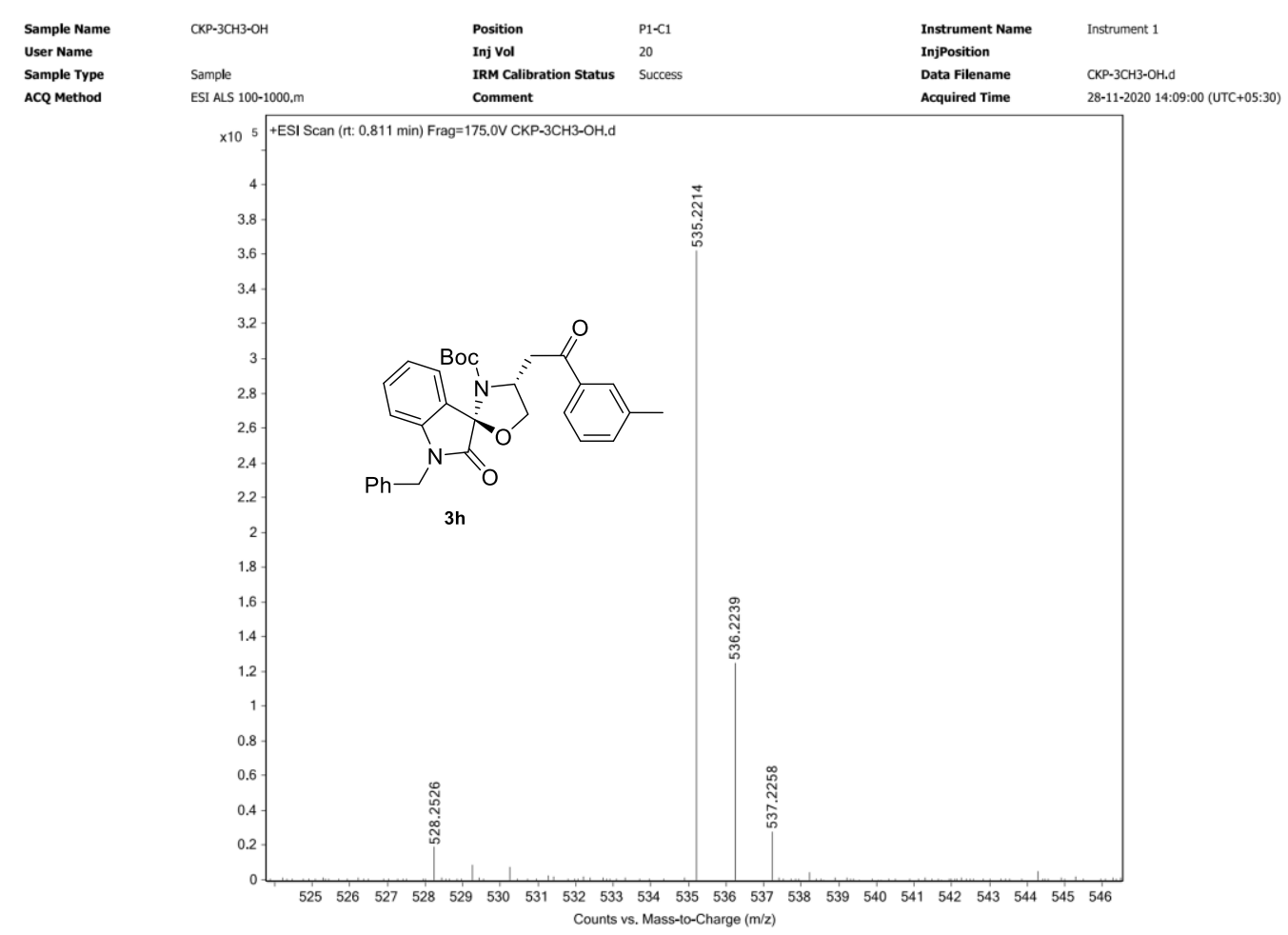


HRMS (ESI) m/z: [M + Na] ${ }^{+}$for $3 \mathbf{i ~} \mathrm{C}_{31} \mathrm{H}_{32} \mathrm{~N}_{2} \mathrm{O}_{5} \mathrm{Na}$ 535.2203, found 535.2211.



HRMS (ESI) m/z: [M + Na $]^{+}$calcd. for 3j $\mathrm{C}_{28} \mathrm{H}_{28} \mathrm{~N}_{2} \mathrm{O}_{5} \mathrm{SNa} 527.1611$, found 527.1613.

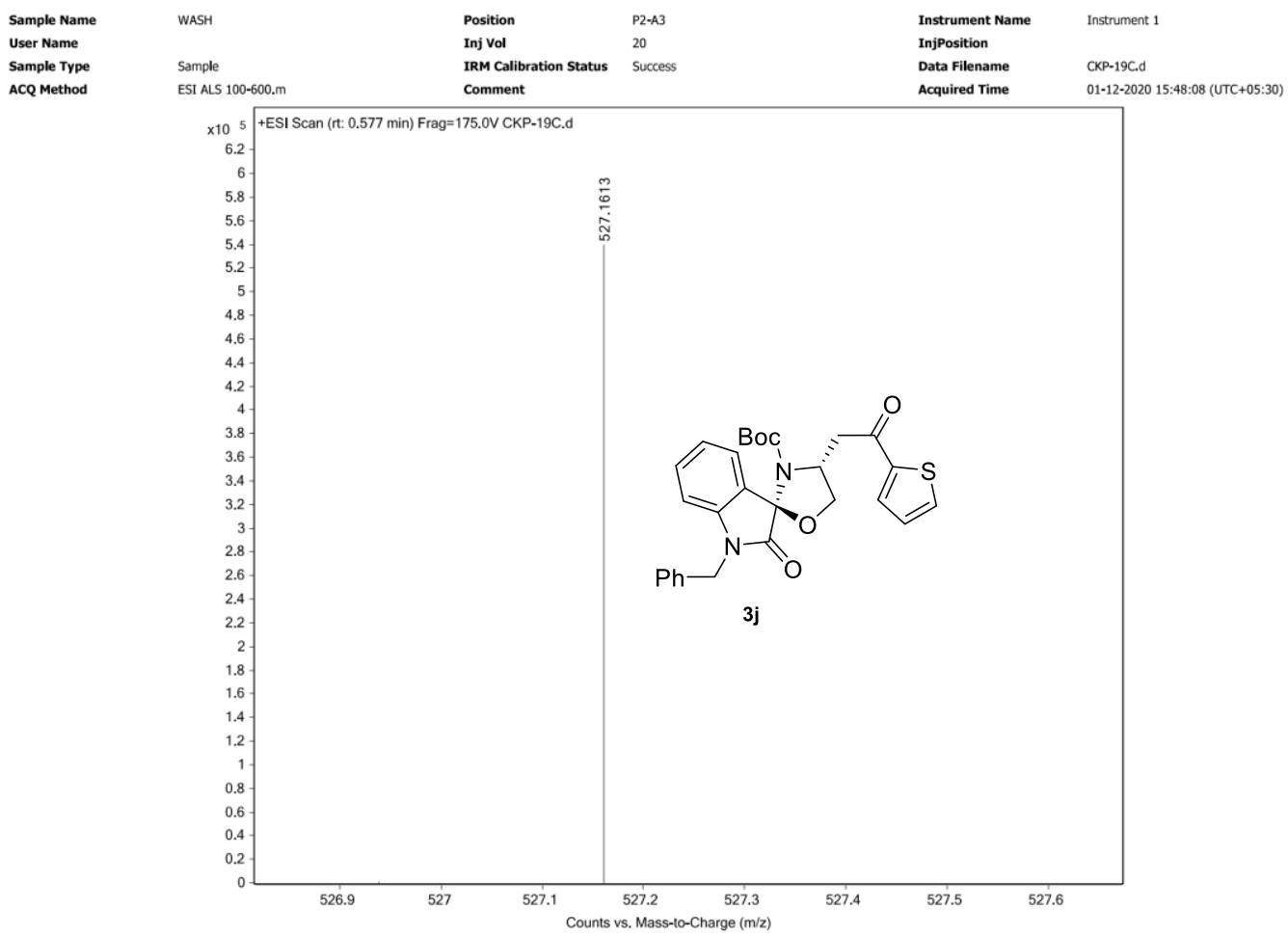


HRMS (ESI) m/z: [M + Na $]^{+}$calcd. for 3k $\mathrm{C}_{32} \mathrm{H}_{34} \mathrm{~N}_{2} \mathrm{O}_{5} \mathrm{Na} 549.2360$, found 549.2360.

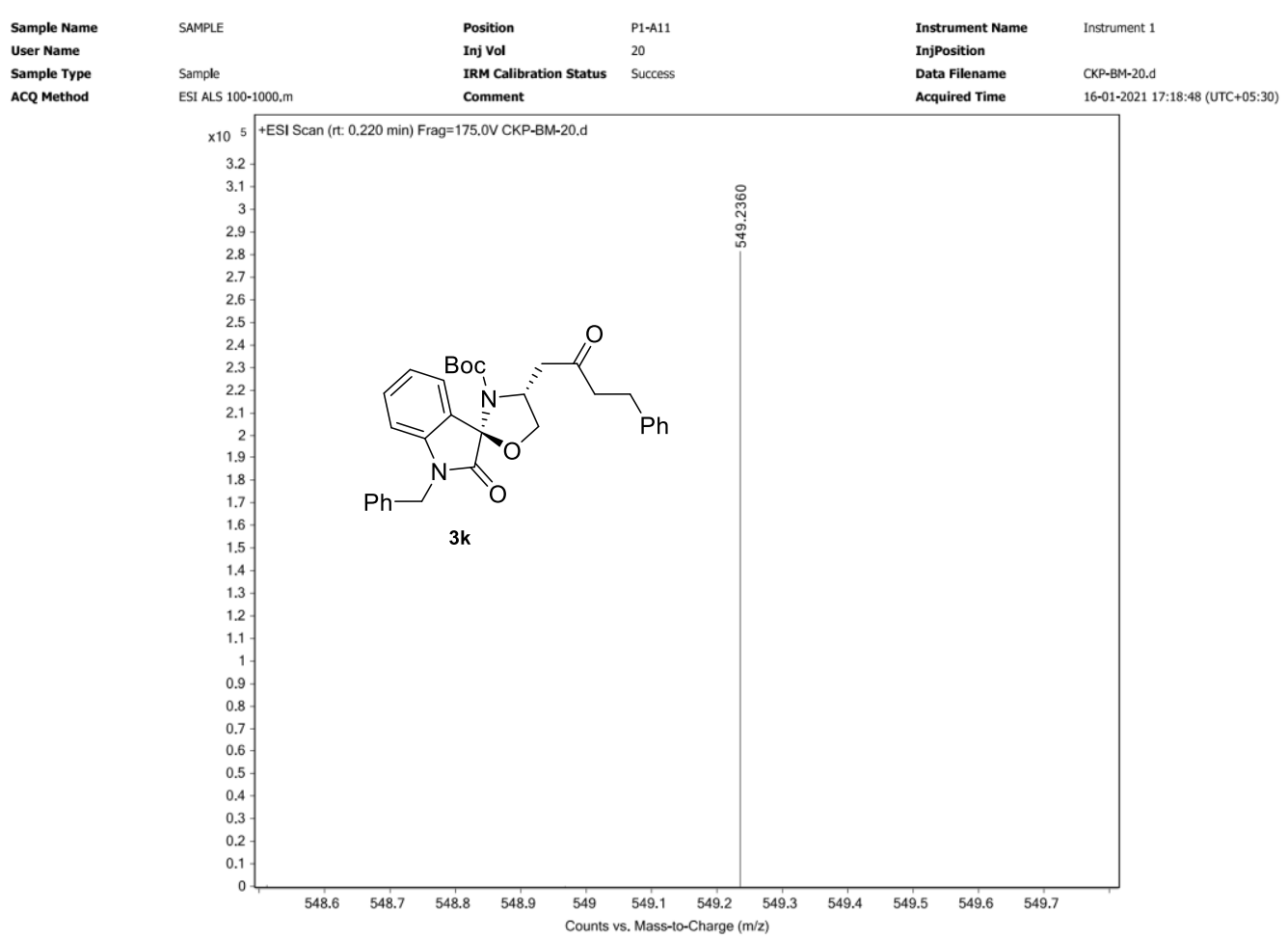

HRMS (ESI) m/z: [M + Na $]^{+}$calcd. for 3 I $\mathrm{C}_{31} \mathrm{H}_{32} \mathrm{~N}_{2} \mathrm{O}_{6} \mathrm{Na} 551.2153$ found 551.2152.

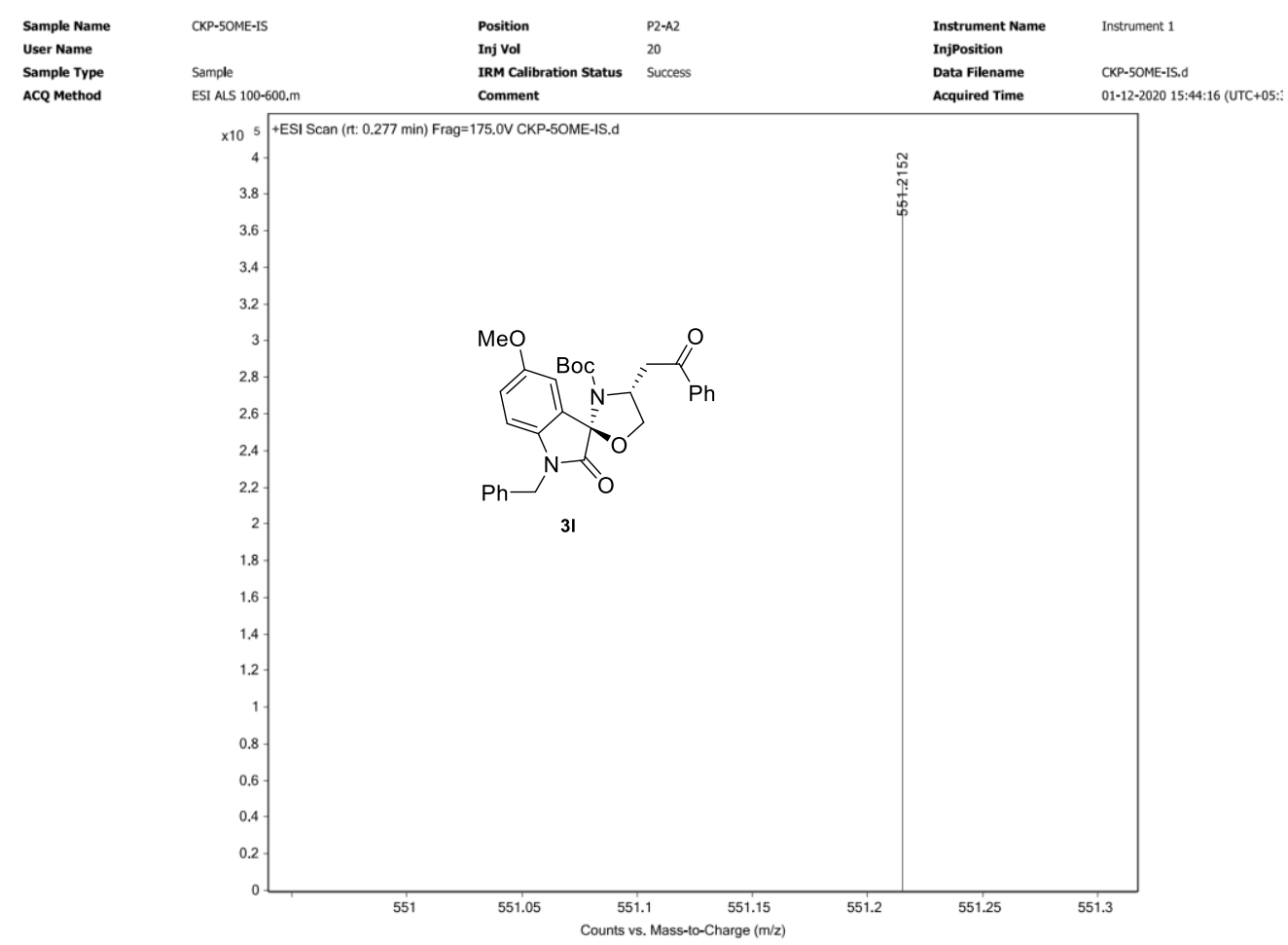


HRMS (ESI) m/z: $[\mathrm{M}+\mathrm{Na}]^{+}$calcd. for $3 \mathrm{~m} \mathrm{C}_{31} \mathrm{H}_{32} \mathrm{~N}_{2} \mathrm{O}_{5} \mathrm{Na} 535.2203$ found 535.2208.



HRMS (ESI) m/z: [M + Na] ${ }^{+}$calcd. for $3 \mathbf{n} \mathrm{C}_{30} \mathrm{H}_{29} \mathrm{FN}_{2} \mathrm{O}_{5} \mathrm{Na} 539.1953$ found 539.1955.




HRMS (ESI) m/z: $[\mathrm{M}+\mathrm{Na}]^{+}$calcd. for $30 \mathrm{C}_{30} \mathrm{H}_{29} \mathrm{ClN}_{2} \mathrm{O}_{5} \mathrm{Na} 555.1657$ found 555.1655.



HRMS (ESI) m/z: [M + Na $]^{+}$calcd. for 3p $\mathrm{C}_{30} \mathrm{H}_{29} \mathrm{BrN}_{2} \mathrm{O}_{5} \mathrm{Na} 599.1152$ found 599.1152.




HRMS (ESI) m/z: [M + Na] ${ }^{+}$calcd. for 3q $\mathrm{C}_{30} \mathrm{H}_{29} \mathrm{ClN}_{2} \mathrm{O}_{5} \mathrm{Na} 555.1657$ found 599.1662.



HRMS (ESI) m/z: $[\mathrm{M}+\mathrm{Na}]^{+}$calcd. for $3 \mathbf{r} \mathrm{C}_{24} \mathrm{H}_{26} \mathrm{~N}_{2} \mathrm{O}_{5} \mathrm{Na} 445.1734$ found 445.1733 .

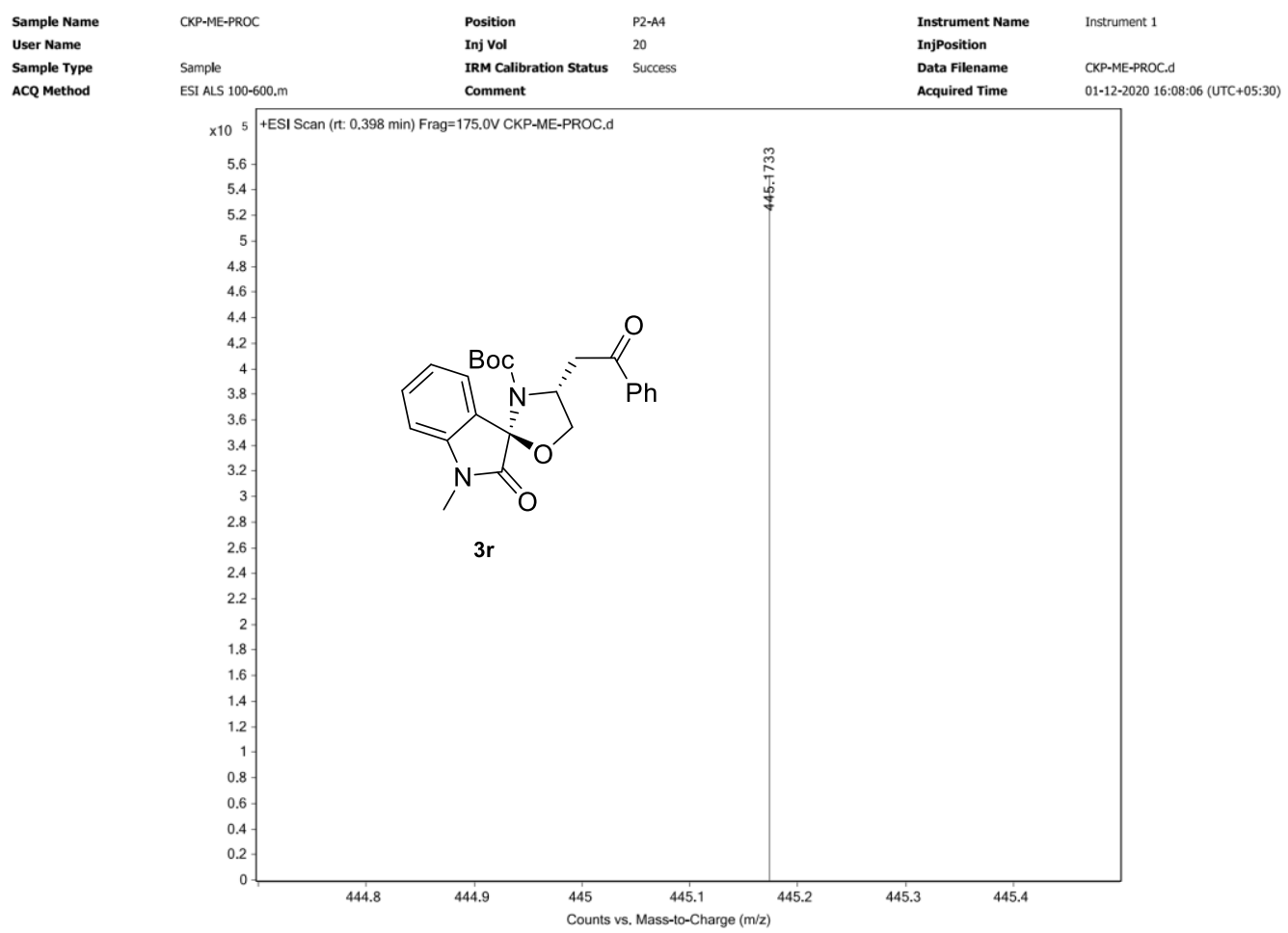


HRMS (ESI) m/z: [M + Na $]^{+}$calcd. for $3 \mathrm{~s} \mathrm{C}_{25} \mathrm{H}_{28} \mathrm{~N}_{2} \mathrm{O}_{5} \mathrm{Na} 459.1890$ found 459.1890 .



HRMS (ESI) m/z: [M + Na $]^{+}$calcd. for $3 t \mathrm{C}_{26} \mathrm{H}_{30} \mathrm{~N}_{2} \mathrm{O}_{5} \mathrm{Na} 473.2047$ found 473.2047.




HRMS (ESI) m/z: [M + Na $]^{+}$calcd. for $4 \mathrm{C}_{30} \mathrm{H}_{32} \mathrm{~N}_{2} \mathrm{O}_{5} \mathrm{Na} 523.2203$ found 523.2207.

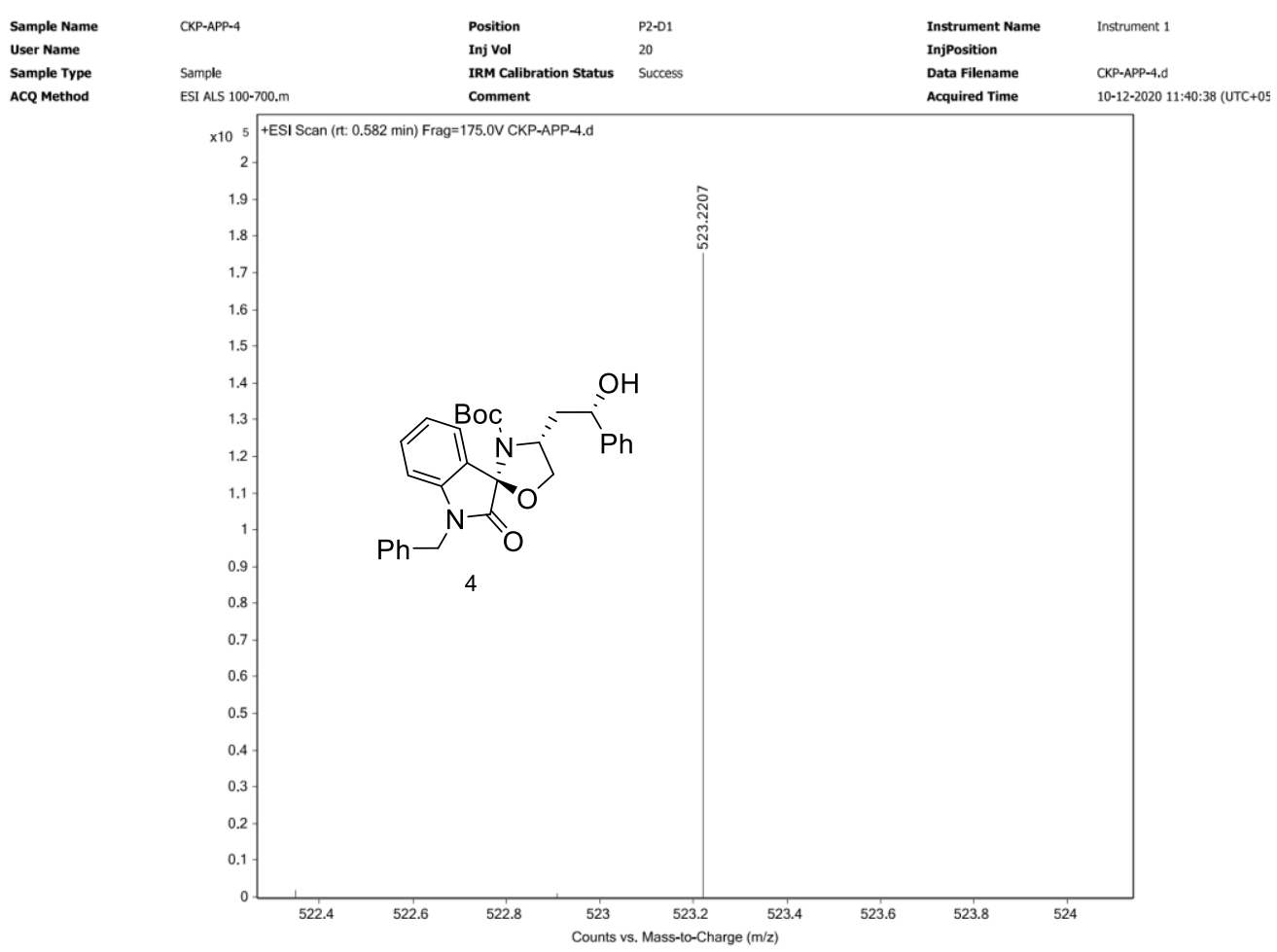

HRMS (ESI) m/z: [M + Na $]^{+}$calcd. for $5 \mathrm{C}_{30} \mathrm{H}_{31} \mathrm{BrN}_{2} \mathrm{O}_{5} \mathrm{Na} 601.1309$ found 601.1358.




HRMS (ESI) m/z: [M + Na] $]^{+}$calcd. for $\mathrm{C}_{38} \mathrm{H}_{34} \mathrm{~N}_{2} \mathrm{O}_{5} \mathrm{Na} 621.2360$ found 621.2360 .



